\title{
Water-Quality Data for Nutrients, Pesticides, and Volatile Organic Compounds in Near-Surface Aquifers of the Midcontinental United States, 1992-1994
}

By Dana W. Kolpin, Kathryn E. Zichelle, and E. Michael Thurman

U.S. GEOLOGICAL SURVEY

Open-File Report 96-435

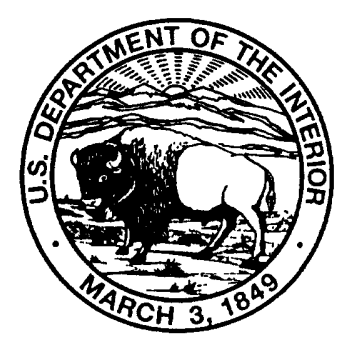




\title{
U.S. DEPARTMENT OF THE INTERIOR \\ BRUCE BABBITT, Secretary
}

\author{
U.S. GEOLOGICAL SURVEY
}

Gordon P. Eaton, Director

The use of firm, trade, and brand names in this report is for identification purposes only and does not constitute endorsement by the U.S. Geological Survey

For addtional information write to:

District Chief

U.S. Geological Survey

Rm. 269, Federal Building

400 South Clinton Street

lowa City, IA 52244
Copies of this report can be purchased from:

U.S. Geological Survey

Branch of Information Services

Box 25286

Federal Center

Denver, CO 80225 


\section{CONTENTS}

Page

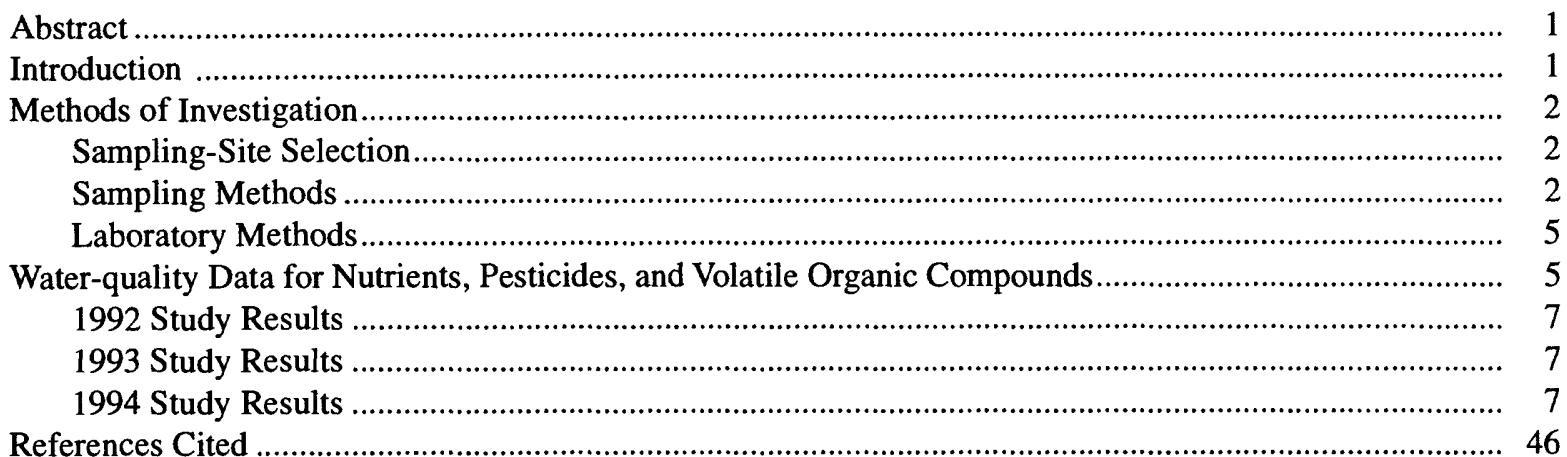

\section{ILLUSTRATIONS}

Figure 1. Map showing the locations of wells sampled in Iowa, Kansas, Minnesota, Missouri, Nebraska, North Dakota, and South Dakota during 1992-1994

2. Map showing the locations of wells sampled in Illinois, Indiana, Michigan, Ohio, and Wisconsin during 1992-1994

3. Graph showing the number of pesticide compounds detected per well for sites sampled from the Midcontinental United States, 1992-1994.

4. Map showing the locations of wells sampled during 1992-1994 and the corresponding number of pesticide compounds detected

\section{TABLES}

Table 1. Pesticide compounds detected in ground-water samples from the Midcontinental United States, 1992-1994

2. Water-quality data from field measurements and laboratory analyses for inorganic nutrients, 1992

3. Water-quality data from routine laboratory analyses of water samples for selected herbicides and herbicide metabolites, 1992

4. Water-quality data from sensitive analytical methods of water samples for selected pesticides and pesticide metabolites, 1992

5. Water-quality data from laboratory analyses of water samples for selected chlorophenoxy-acid and other miscellaneous herbicides, 1992

6. Water-quality data from laboratory analyses of water samples for detected volatile organic compounds and methylene blue active substances, 1992 .

7. Water-quality data from field measurements and laboratory analyses for inorganic nutrients, 1993

8. Water-quality data from laboratory analyses of water samples for selected herbicides and herbicide metabolites, 1993

9. Water-quality data from laboratory analyses of water samples for selected volatile organic compounds for wells with reported detections, 1993 


\section{TABLES, Continued}

10. Water-quality data from field measurements and laboratory analyses for inorganic nutrients, 1994

11. Water-quality data from laboratory analyses of water samples for selected herbicides and herbicide metabolites, 1994 


\section{CONVERSION FACTORS AND ABBREVIATED WATER-QUALITY UNITS}

\begin{tabular}{lcl}
\hline Multiply & By & To obtain \\
\hline foot & 0.3048 & meter $(\mathrm{m})$ \\
gallon & 3.785 & liter $(\mathrm{L})$ \\
ounce & 28.353 & gram $(\mathrm{g})$
\end{tabular}

Degrees Celsius $\left({ }^{\circ} \mathrm{C}\right)$ may be converted to degrees Fahrenheit $\left({ }^{\circ} \mathrm{F}\right)$ by using the following formula:

$$
{ }^{\circ} \mathrm{F}=9 / 5\left({ }^{\circ} \mathrm{C}\right)+32 \text {. }
$$

Milligram per liter $(\mathrm{mg} / \mathrm{L})$ is a unit expressing the concentration of a chemical constituent in solution as weight (milligrams) of solute per unit volume (liter) of water.

Microgram per liter $(\mu \mathrm{g} / \mathrm{L})$ is a unit expressing the concentration of a chemical constituent in solution as weight (micrograms) of solute per unit volume (liter) of water. 


\title{
Water-Quality Data for Nutrients, Pesticides, and Volatile Organic Compounds in Near-Surface Aquifers of the Midcontinental United States, 1992-1994
}

\author{
By Dana W. Kolpin, Kathryn E. Zichelle, and E. Michael Thurman
}

\section{ABSTRACT}

Water samples were collected from 175 wells in 12 Midcontinental States (Illinois, Indiana, Iowa, Kansas, Michigan, Minnesota, Missouri, Nebraska, North Dakota, Ohio, South Dakota, Wisconsin) from 1992 through 1994 to determine the spatial distribution of nutrients, pesticides, and volatile organic compounds in ground water, and to document the potential effects of the historic flooding that occurred during 1993 on ground-water quality.

Concentrations of nitrate greater than the $0.05 \mathrm{mg} / \mathrm{L}$ reporting limit were found in 69.1 percent of the water samples, and nitrate concentrations exceeded the U.S. Environmental Protection Agency maximum contaminant limit of $10 \mathrm{mg} / \mathrm{L}$ in 9.6 percent of the 249 samples analyzed for nitrate. Pesticides or pesticide metabolites were detected in 72.4 percent of the 210 pesticide analyses, and 28 different compounds were found. Concentrations of multiple pesticide compounds above analytical reporting limits were found in water from about 60 percent of the wells sampled. Although pesticides were frequently detected, only one sample had a pesticide concentration that exceeded a maximum contaminant level for drinking water. The most frequently detected compounds, however, were pesticide metabolites for which maximum contaminant levels have not yet been established. Volatile organic compounds were detected in 13.5 percent of the 155 samples analyzed for these compounds. Only one sample had concentrations of volatile organic compounds that exceeded a maximum contaminant level for drinking water.

\section{INTRODUCTION}

This report is one in a series of water-quality reports that present the analytical results from regional studies conducted by the U.S. Geological Survey (USGS) that investigate the movement and distribution of agricultural chemicals in the water resources of the Midcontinental United States. Previous published data in the series report the analytical results from regional studies of ground water (Kolpin, Burkart, and Thurman, 1993), surface water (Scribner and others, 1993), storm runoff (Scribner and others, 1994), and precipitation (Goolsby and others, 1995).

The Midcontinental United States (the region comprised of Illinois, Indiana, Iowa, Kansas, Michigan, Minnesota, Missouri, Nebraska, North Dakota, Ohio, South Dakota, and Wisconsin) is the largest and most intensive crop producing region of the country. This region accounts for about 60 percent of the Nation's pesticide use (Gianessi and Puffer, 1990; 1992) and inorganic fertilizer use (U.S. Environmental Protection Agency, 1990), although it makes up only about 21 percent of the Nation's land surface. More than 29 million people in the Midcontinent rely on ground water as a source of drinking water (U.S. Geological Survey, 1985). Thus, the intensive application of agricultural chemicals to the land surface has created concern about nonpoint-source contamination of the region's ground-water resources.

To better understand the movement and distribution of agricultural chemicals in ground water, the USGS designed a reconnaissance network that was geographically and hydrogeologically representative 
of near-surface aquifers in the corn- and soybean-producing region of the Midcontinental United States (Kolpin and Burkart, 1991). The objectives of this study were to (1) determine the hydrogeologic, spatial, and seasonal distribution of agricultural chemicals in near-surface aquifers in the Midcontinental United States, (2) determine the statistical relations of agricultural chemicals to natural and anthropogenic factors, and (3) obtain data on agricultural chemical concentrations in ground water from geographic areas where few data existed. Near-surface aquifers were defined as having the top of the aquifer material within about 15 $m$ of land surface.

The first part of the study began in 1991. A random approach was used to select 303 wells from which water samples were collected in March or April (preplanting) and July or August (postplanting) 1991. Measurable concentrations of herbicides or triazine metabolites were detected in 28.4 percent of the 303 wells sampled during the 1991 study. However, concentrations of these compounds did not exceed maximum contaminate levels or health advisory levels (U.S. Environmental Protection Agency, 1992) for drinking water. The results of the 1991 study showed that water from near-surface unconsolidated aquifers is more likely to contain both pesticides and nitrate (nitrate plus nitrite, hereafter referred to as "nitrate") than water from near-surface bedrock aquifers (Kolpin and others, 1994). A complete description of the interpretive and analytical results for the 1991 study can be found elsewhere (Burkart and Kolpin, 1993; Kolpin and others, 1994; Kolpin, Burkart, and Thurman, 1993).

Additional ground-water samples were collected from 1992 through 1994 to continue the ongoing investigation of the regional distribution of agricultural chemicals in ground water. The objectives of the 1992 sampling and analyses were to determine the relations of analytical reporting limits, the number of parent compounds present, and the number of metabolites to the frequency of pesticide detection. The objective of the 1993 sampling and analyses was to determine whether the 1993 flooding had immediate effects on the water quality of near-surface unconsolidated aquifers in the upper Mississippi River Basin. The objective of the 1994 sampling and analyses was to determine if there had been long-term effects of the 1993 flood on the water quality of near-surface unconsolidated aquifers underlying areas in the upper Mississippi River Basin that had been severely flooded. Funding for the collection and analysis of water samples from 38 wells during the 1994 postplanting season was provided by the U.S. Environmental Protection Agency (USEPA).

The purpose of this report is to present the waterquality data collected from the ground-water monitoring network during July through August 1992, September through October 1993, and July through August 1994.

\section{METHODS OF INVESTIGATION}

\section{Sampling-Site Selection}

In 1992, 101 wells were sampled during July or August after the application of pesticides. These 101 wells were randomly selected by a process that ensured a geographic distribution by State and hydrogeologic distribution by aquifer class (unconsolidated or bedrock) across the Midcontinent (Kolpin, Goolsby, and others, 1993; Kolpin and others, 1995).

In 1993, 110 wells were sampled during September or October. Wells selected for the 1993 sampling were completed in unconsolidated aquifers, contained post-1953 water (determined from analysis of tritium concentrations), and were located in the region of the Midcontinent that had more than 150 percent of normal rainfall from April 1 to July 31, 1993 (Kolpin and Thurman, 1995).

In 1994, 38 wells were sampled during July or August. These wells were a subset of the wells sampling during 1993 and were located in areas that were noted to have the worst flooding problems during the 1993 sampling (Kolpin, Nations, and others, 1996).

\section{Sampling Methods}

The locations of the 175 wells sampled from the ground-water reconnaissance network since 1992 are shown in figures 1 and 2. The methods used to collect and process the water samples were identical to those for the 1991 study (Kolpin and Burkart, 1991). All of the samples were collected by USGS personnel with equipment constructed of materials, such as glass and stainless steel, that would not leach or adsorb organic compounds. Decontamination procedures were implemented to prevent cross-contamination of water between wells and samples. Wells were purged before sampling until $\mathrm{pH}$, water temperature, and specific conductance stabilized. Where possible, water levels were measured before purging. 


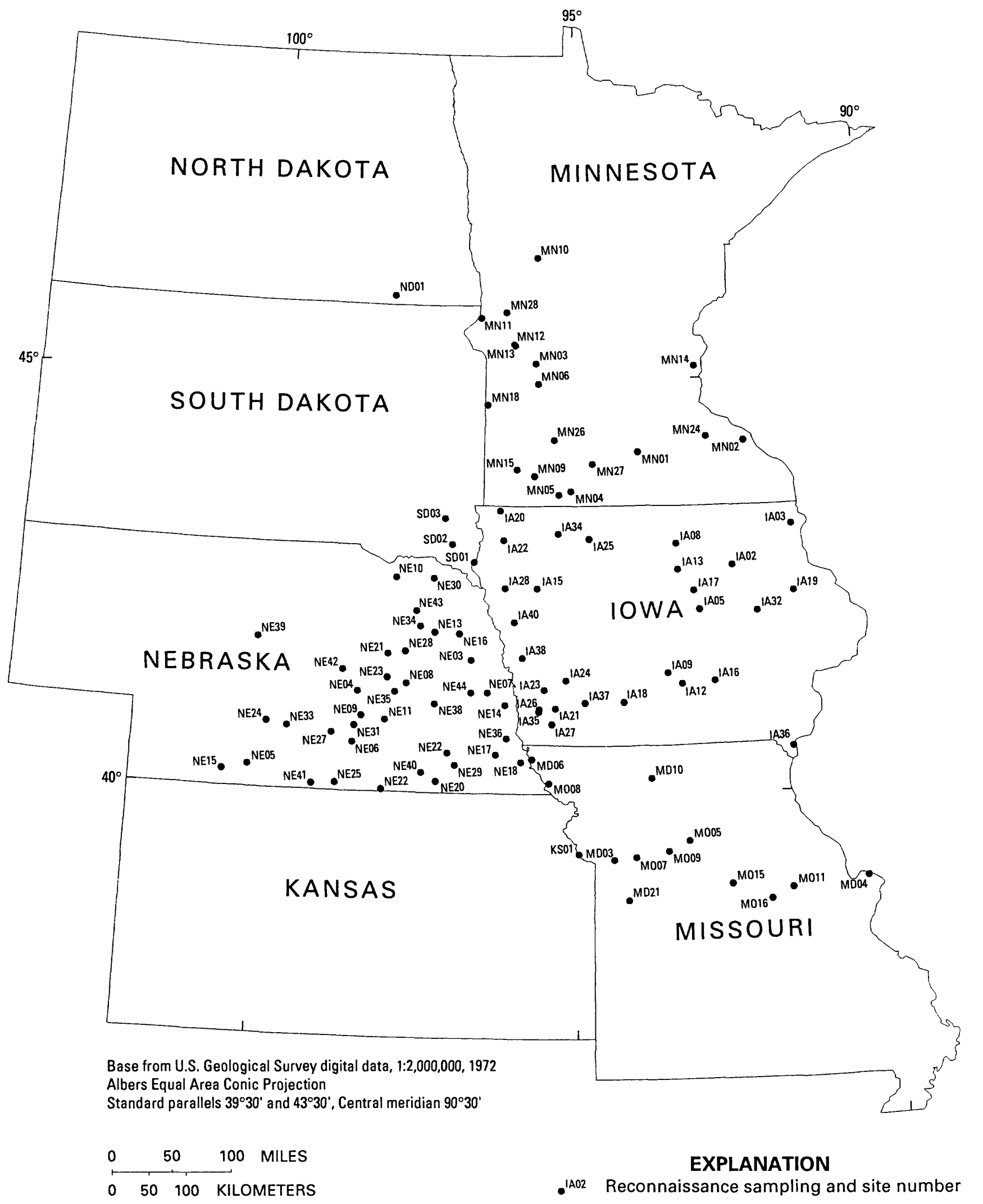

Figure 1. Locations of wells sampled in lowa, Kansas, Minnesota, Missouri, Nebraska, North Dakota, and South Dakota during 1992-94. 

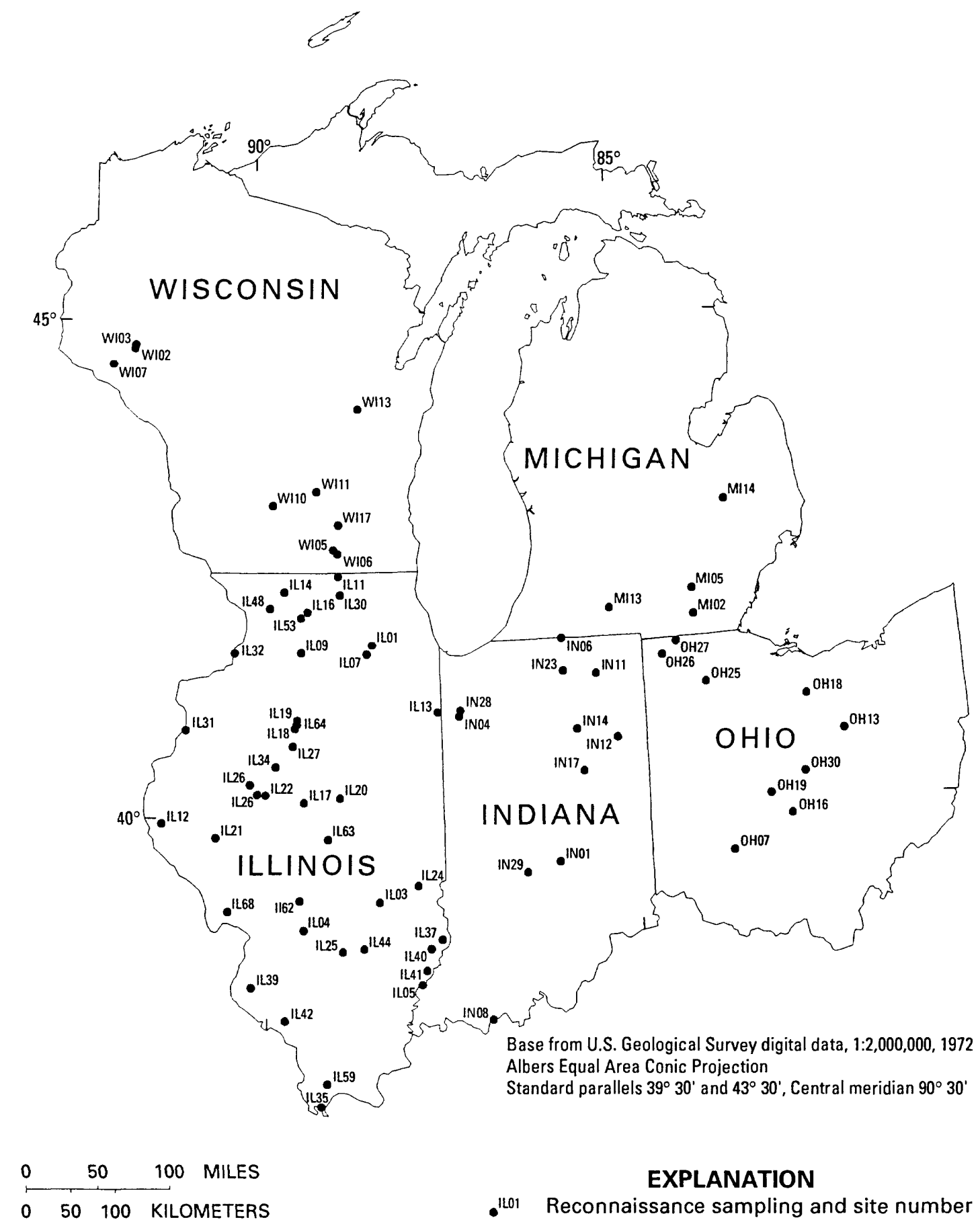

EXPLANATION

- Ro1 Reconnaissance sampling and site number

Figure 2. Locations of wells sampled in Illinois, Indiana, Michigan, Ohio, and Wisconsin during $1992-94$. 


\section{Laboratory Methods}

A routine analytical method by gas chromatography/mass spectrometry (GC/MS) following solidphase extraction on C-18 cartridges (Thurman and others, 1990; Meyer and others, 1993) was used to obtain concentrations of selected herbicides and triazine metabolites. The analytical reporting limit for this method was $0.05 \mu \mathrm{g} / \mathrm{L}$ for all compounds.

To quantify concentrations less than $0.05 \mu \mathrm{g} / \mathrm{L}$, analytes were extracted by a solid-phase extraction method from 1-liter water samples (Zaugg and others, 1995). This method used disposable polypropylene cartridges containing $0.5 \mathrm{~g}$ of porous silica coated with a chemically bonded, octydecyl (C-18) phase. Chlorophenoxy-acid herbicides were analyzed by methods described in Wershaw and others (1987). An alachlor metabolite, alachlor ethanesulfonic acid (alachlorESA), was isolated by solid-phase extraction and analyzed by enzyme-linked immunosorbent assay (Aga and others, 1994). In addition, cyanazine metabolites (cyanazine amide, deethylcyanazine amide, and deethylcyanazine) were isolated and analyzed by a method of solid-phase extraction and GC/MS (Meyer, 1994).

Concentrations of selected inorganic nutrients were determined by colorimetric methods (Fishman and Friedman, 1989). Concentrations of 63 volatile organic compounds were analyzed in unacidified water samples according to USEPA method 524.2. Methylene blue active substances (MBAS), which is an indicator measurement for anionic surfactants, were determined by extraction into chloroform, backwashed with an acidified phosphate-based buffer solution, and measured against external standards with a probe spectrophotometer (Burkhardt and others, 1995). The analytical reporting limit for this method was $0.01 \mathrm{mg} / \mathrm{L}$.

\section{WATER-QUALITY DATA FOR NUTRIENTS, PESTICIDES, AND VOLATILE ORGANIC COMPOUNDS}

From 1992 to 1994,249 water samples were collected from 175 wells in the ground-water reconnaissance network. Hydrogeologic, well construction, land-use, and locational information for these wells is published elsewhere (Kolpin and others, 1993). Concentrations of nitrate greater than the $0.05 \mathrm{mg} / \mathrm{L}$ analytical reporting limit were found in 69.1 percent of the 249 samples analyzed for nutrients; nitrate in 9.6 percent of the samples exceeded the USEPA maximum contaminant level (MCL) of $10.0 \mathrm{mg} / \mathrm{L}$.
Concentrations of pesticides or pesticide metabolites at or greater than analytical reporting limits were found in 72.4 percent of the 210 samples analyzed for pesticides. The frequencies of detection for the 28 pesticides and pesticide metabolites found in ground water during 1992-94 are listed in table 1 (p. 8). Although pesticides were frequently detected, only one sample had a pesticide concentration that exceeded a USEPA MCL for drinking water. However, pesticide metabolites were the most frequently detected compounds during the 1992-94 sampling. Thus, pesticide metabolites are prevalent in ground water and, in many cases, are more persistent and mobile than the parent compound (Kolpin, Thurman, and Goolsby, 1996). For example, alachlor-ESA was found about 10 times as frequently and at much higher concentrations than alachlor itself (table 1). Consequently, if herbicide metabolites are not quantified, the effects of herbicide use on ground-water quality can be substantially underestimated. Drinking-water standards have yet to be determined for the metabolites examined for this study.

Multiple pesticide compounds commonly were found in a single water sample (figures 3 and 4). The potential synergistic effects to human health and the aquatic environment from the presence of multiple pesticides compounds are not yet clearly understood. Water in about 60 percent of the wells sampled contained more than one pesticide compound, and as many as 13 different compounds were detected in a single well (fig. 3).

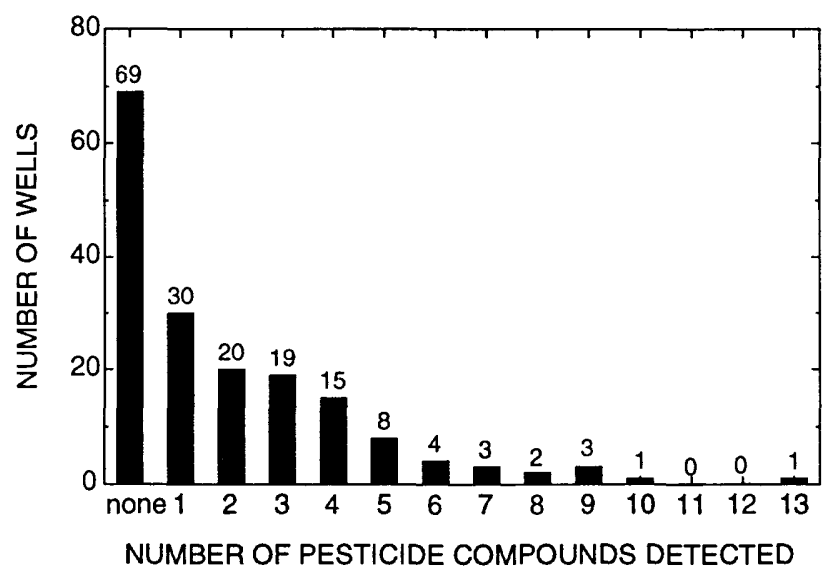

Figure 3. Number of pesticide compounds detected per well for sites sampled from the Midcontinental United States, 1992-94. 


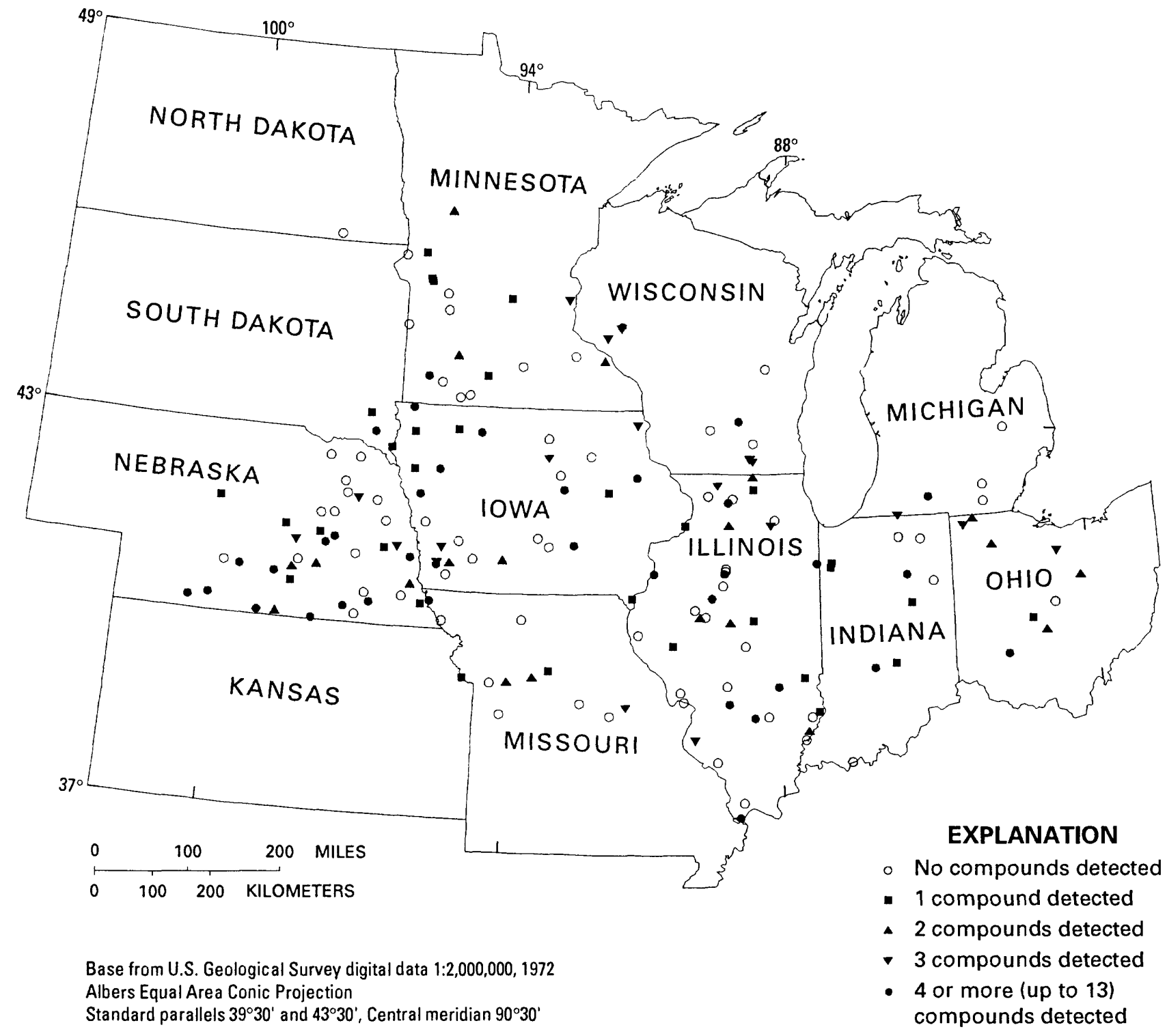

Figure 4. Locations of wells sampled during 1992-94, and the corresponding number of pesticide compounds detected. 
Volatile organic compounds were detected in 13.5 percent of the 155 samples analyzed for these compounds. Only one sample had concentrations of volatile organic compounds that exceeded USEPA MCL's for drinking water.

\section{Study Results}

Water samples collected from 101 wells during July or August 1992 were analyzed for concentrations of 4 inorganic nutrients (table 2, p. 10), 66 pesticide or pesticide metabolites (many with analytical reporting limits as low as $0.002 \mu \mathrm{g} / \mathrm{L}$ ) (tables 3-5, p. 15-26), and 63 volatile organic compounds (table 6, p. 27). During this study, nitrate concentrations equalled or exceeded $3.0 \mathrm{mg} / \mathrm{L}$ in 32.7 percent of the samples, and nitrate concentrations exceeded the USEPA MCL in water from 7.9 percent of the wells sampled. Pesticides or pesticide metabolites were detected in water from 62 percent of the samples collected in 1992. This frequency of detection is substantially greater than the 24 percent determined from 579 samples collected for the previous (1991) study of the reconnaissance network (Kolpin and others, 1994). The greater frequency of detection during 1992 was primarily due to the increased number of pesticide metabolites examined and the much lower analytical reporting limits that were available (Kolpin and others, 1995). The frequency of pesticide detection was inversely related to the analytical reporting limit. A monotonic increase was determined in the frequency of detection as analytical reporting limits are decreased (Kolpin and others, 1995; Kolpin and Goolsby, 1995). For example, the number of atrazine detections decreases by about onehalf as the reporting limit increases from $0.01 \mu \mathrm{g} / \mathrm{L}$ to $0.10 \mu \mathrm{g} / \mathrm{L}$. At least one volatile organic compound was detected in 11.1 percent ( 5 of 45 wells) of the analyses. Chloroform was the most frequently detected volatile organic compound in samples collected during 1992 (table 6).

\section{Study Results}

Water samples collected from 110 wells from September to October 1993 were analyzed for concentrations of 4 inorganic nutrients (table 7, p. 29), 14 pesticides or pesticide metabolites (table 8, p. 34), and 63 volatile organic compounds (table 9, p. 39). Nitrate concentrations exceeded the MCL of $10 \mathrm{mg} / \mathrm{L}$ in water from 10 percent of the sampled wells. Pesticides or pesticide metabolites were detected in water from 55.4 percent of the wells; alachlor-ESA was the most frequently detected compound. One sample contained a concentration of alachlor that exceeded the MCL of 2.0 $\mu \mathrm{g} / \mathrm{L}$ for treated drinking water (table 8). Several previous water samples from this well exceeded one-half of the MCL for alachlor (table 3) (Kolpin, Burkart, and Thurman, 1993). Water from 14.5 percent of the wells contained at least one of the 63 volatile organic compounds for which samples were analyzed for this study. Only 1,2-dichloroethane and benzene concentrations in one sample exceeded a USEPA MCL $(5.0 \mu \mathrm{g} / \mathrm{L})$ for treated drinking water. Significantly more urban residential and industrial land use was within a 30-meter radius of wells where volatile organic compounds were detected (Kolpin and Thurman, 1995).

The historic 1993 flooding affected groundwater quality. There is direct relation between increases in total herbicide concentration (between the preflood and postflood sample) and the occurrence of stream flooding near a well (Kolpin and Thurman, 1995). About 63 percent of the wells that had at least a 20 -percent increase in total herbicide concentration in water samples were located in areas that also were severely affected by stream flooding. An inverse relation was determined between well depth and changes in total herbicide concentration (Kolpin and Thurman, 1995). Water in shallow wells more quickly reflects changes in water quality in response to changes in recharge from the 1993 flooding.

\section{Study Results}

Water samples were collected from 38 wells sampled during July to August 1994 and analyzed for concentrations of 4 inorganic nutrients (table 10, p. 41) and 18 pesticides and pesticide metabolites (table 11, p. 43). Nitrate concentrations exceeded the MCL of 10 $\mathrm{mg} / \mathrm{L}$ in water from 13.2 percent of the sampled wells. Pesticides or pesticide metabolites were detected in water from 76.3 percent of the wells. However, no pesticide concentrations exceeded USEPA MCL's for treated drinking water. Alachlor-ESA again was the most frequently detected pesticide compound found in the samples taken during 1994. No acetochlor concentrations above the analytical reporting limit of 0.05 $\mu \mathrm{g} / \mathrm{L}$ were reported. Acetochlor is an herbicide used with corn crops, and it was first registered by the USEPA for use during 1994. Possible explanations for the absence of acetochlor in ground water in the 1994 samples include the rapid degradation of acetochlor in the soil zone, insufficient time for this first extensive use of acetochlor to have reached the aquifers sampled, and the possible lack of acetochlor use in the recharge areas for the wells sampled (Kolpin, Nations, and others, 1996). 
Table 1. Pesticide compounds detected in ground-water samples from the Midcontinental United States, 1992-94

[Alachlor-ESA, ethanesulfonic acid; sensitive, results of analyses using lower reporting limits; $\mu \mathrm{g} / \mathrm{L}$, micrograms per liter; Max, maximum; conc., concentrations; MCL, maximum contaminant level; HAL, health advisory level, —, no data]

\begin{tabular}{|c|c|c|c|c|c|c|c|c|}
\hline Compound & $\begin{array}{c}\text { Percent } \\
\text { detection }\end{array}$ & $\begin{array}{l}\text { Number } \\
\text { of samples }\end{array}$ & $\begin{array}{l}\text { Report- } \\
\text { ing limit } \\
(\mu g / L)\end{array}$ & $\begin{array}{l}\text { Max } \\
\text { conc. } \\
(\mu g / L)\end{array}$ & $\begin{array}{l}\text { Median } \\
\text { reported } \\
\text { conc. } \\
(\mu g / L)\end{array}$ & $\begin{array}{c}\text { MCL } \\
(\mu \mathrm{g} / \mathrm{L})\end{array}$ & $\begin{array}{c}\text { HAL } \\
(\mu g / L)\end{array}$ & Use or origin \\
\hline $\begin{array}{l}\text { Alachlor- } \\
\text { ESA }\end{array}$ & 46.7 & 214 & .10 & 8.63 & .665 & - & - & $\begin{array}{l}\text { Herbicide metabolite } \\
\quad \text { (alachlor) }\end{array}$ \\
\hline $\begin{array}{l}\text { Atrazine } \\
\text { (sensitive) }\end{array}$ & 37.2 & 94 & .003 & .84 & .035 & 3 & 3 & Herbicide \\
\hline $\begin{array}{l}\text { Deethyl- } \\
\text { atrazine }\end{array}$ & 27.1 & 214 & .05 & 1.79 & .135 & - & - & $\begin{array}{l}\text { Triazine metabolite } \\
\text { (atrazine, pro- } \\
\text { pazine) }\end{array}$ \\
\hline Atrazine & 26.2 & 214 & .05 & 1.8 & .215 & 3 & 3 & Herbicide \\
\hline $\begin{array}{l}\text { Deethyl- } \\
\text { atrazine } \\
\text { (sensitive) }\end{array}$ & 25.5 & 94 & .002 & .14 & .009 & - & - & $\begin{array}{l}\text { Triazine metabolite } \\
\text { (atrazine, pro- } \\
\text { pazine) }\end{array}$ \\
\hline $\begin{array}{l}\text { 2,6-Diethyl- } \\
\text { analine }\end{array}$ & 16.0 & 94 & .002 & .022 & .003 & - & - & $\begin{array}{l}\text { Herbicide metabolite } \\
\quad \text { (alachlor) }\end{array}$ \\
\hline $\begin{array}{l}\text { DCPA acid } \\
\text { metabolite }\end{array}$ & 15.6 & 45 & .01 & 2.22 & .03 & - & - & $\begin{array}{l}\text { Herbicide metabolite } \\
\text { (dacthal) }\end{array}$ \\
\hline $\begin{array}{l}\text { Simazine } \\
\quad \text { (sensitive) }\end{array}$ & 12.8 & 94 & .002 & .077 & .0035 & 4 & 4 & Herbicide \\
\hline $\begin{array}{l}\text { Deisopropyl- } \\
\text { atrazine }\end{array}$ & 13.6 & 214 & .05 & .48 & .13 & - & - & $\begin{array}{l}\text { Triazine metabolite } \\
\text { (atrazine, cyana- } \\
\text { zine, simazine) }\end{array}$ \\
\hline $\begin{array}{l}\text { Metolachlor } \\
\text { (sensitive) }\end{array}$ & 10.6 & 94 & .002 & .57 & .0185 & - & 1000 & Herbicide \\
\hline $\begin{array}{l}\text { Cyanazine } \\
\text { Amide }\end{array}$ & 9.6 & 104 & .05 & .55 & .16 & - & - & $\begin{array}{l}\text { Herbicide metabolite } \\
\quad \text { (cyanazine) }\end{array}$ \\
\hline Prometon & 8.4 & 214 & .05 & 1.35 & .10 & - & 100 & Herbicide \\
\hline 2,4-D & 6.7 & 45 & .01 & .89 & .17 & 70 & 70 & Herbicide \\
\hline P,P'DDE & 6.4 & 94 & .006 & .03 & .011 & - & - & $\begin{array}{l}\text { Insecticide metabolite } \\
\text { (DDT) }\end{array}$ \\
\hline $\begin{array}{l}\text { Prometon } \\
\quad \text { (sensitive) }\end{array}$ & 6.4 & 94 & .01 & .96 & .055 & - & 100 & Herbicide \\
\hline Metolachlor & 6.1 & 214 & .05 & .76 & .19 & - & 1000 & Herbicide \\
\hline $\begin{array}{l}\text { Alachlor } \\
\text { (sensitive) }\end{array}$ & 5.3 & 94 & .003 & .662 & .079 & 2 & - & Herbicide \\
\hline Dicamba & 4.4 & 45 & .01 & .01 & .01 & - & 200 & Herbicide \\
\hline Picloram & 4.4 & 45 & .01 & .03 & .02 & 500 & 500 & Herbicide \\
\hline Alachlor & 4.2 & 214 & .05 & 4.27 & .23 & 2 & - & Herbicide \\
\hline Chlorpyrifos & 4.2 & 94 & .002 & .024 & .009 & - & 20 & Insecticide \\
\hline
\end{tabular}


Table 1. Pesticide compounds detected in ground-water samples from the Midcontinental United States, 1992-94-Continued

\begin{tabular}{|c|c|c|c|c|c|c|c|c|}
\hline Compound & $\begin{array}{l}\text { Percent } \\
\text { detection }\end{array}$ & $\begin{array}{l}\text { Number } \\
\text { of samples }\end{array}$ & $\begin{array}{c}\text { Report- } \\
\text { ing limit } \\
(\mu g / L)\end{array}$ & $\begin{array}{l}\text { Max } \\
\text { conc. } \\
(\mu g / L)\end{array}$ & $\begin{array}{c}\text { Median } \\
\text { reported } \\
\text { conc. } \\
(\mu \mathrm{g} / \mathrm{L})\end{array}$ & $\begin{array}{c}M C L \\
(\mu g / L)\end{array}$ & $\begin{array}{c}\text { HAL } \\
(\mu g / L)\end{array}$ & Use or origin \\
\hline Ethalfluralin & 3.2 & 94 & .005 & .014 & .012 & - & - & Herbicide \\
\hline Cyanazine & 2.8 & 214 & .05 & .88 & .115 & - & 1 & Herbicide \\
\hline $2,4,5-\mathrm{T}$ & 2.2 & 45 & .01 & .02 & .02 & - & 70 & Herbicide \\
\hline $\begin{array}{l}\text { Cyanazine } \\
\text { (sensitive) }\end{array}$ & 2.1 & 94 & .01 & .02 & .015 & - & 1 & Herbicide \\
\hline EPTC & 2.1 & 94 & .002 & .003 & .0025 & - & - & Herbicide \\
\hline Trifluralin & 2.1 & 94 & .003 & .016 & .012 & - & 5 & Herbicide \\
\hline Triallate & 2.1 & 94 & .003 & .007 & .0055 & - & - & Herbicide \\
\hline Simazine & 1.9 & 214 & .05 & .09 & .07 & 4 & 4 & Herbicide \\
\hline Metribuzin & 1.4 & 214 & .05 & .22 & .08 & - & 200 & Herbicide \\
\hline Benfluralin & 1.1 & 94 & .005 & .018 & .018 & - & - & Herbicide \\
\hline Napropamide & 1.1 & 94 & .002 & .008 & .008 & - & - & Herbicide \\
\hline $\begin{array}{l}\text { Pendi- } \\
\text { methalin }\end{array}$ & 1.1 & 94 & .008 & .01 & .01 & - & - & Herbicide \\
\hline Tebuthiuron & 1.1 & 94 & .01 & .05 & .05 & - & 500 & Herbicide \\
\hline $\begin{array}{l}\text { Propachlor } \\
\text { (sensitive) }\end{array}$ & 1.1 & 94 & .002 & .002 & .002 & - & 90 & Herbicide \\
\hline $\begin{array}{l}\text { Metribuzin } \\
\text { (sensitive) }\end{array}$ & 1.1 & 94 & .005 & .01 & .01 & - & 200 & Herbicide \\
\hline
\end{tabular}


Table 2. Water-quality data from field measurements and laboratory analyses of water samples for inorganic nutrients, 1992 $\left[{ }^{\circ} \mathrm{C}\right.$, degrees Celsius; $\mu \mathrm{S} / \mathrm{cm}$, microsiemens per centimeter at 25 degrees Celsius; $\mathrm{mg} / \mathrm{L}$, milligrams per liter; - , no data; $<$, less than]

\begin{tabular}{|c|c|c|c|c|c|c|c|c|c|c|}
\hline $\begin{array}{c}\text { Site } \\
\text { identifier } \\
\text { (figs. 1-2) }\end{array}$ & $\begin{array}{c}\text { Date } \\
\text { sampled } \\
\text { (month, } \\
\text { day) }\end{array}$ & $\begin{array}{l}\text { Water } \\
\text { level } \\
\text { (feet) }\end{array}$ & $\begin{array}{c}\text { Water } \\
\text { temp- } \\
\text { perature } \\
\left({ }^{\circ} \mathrm{C}\right)\end{array}$ & $\begin{array}{c}\text { Specific } \\
\text { conduct- } \\
\text { ance } \\
(\mu \mathrm{S} / \mathrm{cm})\end{array}$ & $\begin{array}{l}\mathrm{pH} \\
\text { (stan- } \\
\text { dard } \\
\text { units) }\end{array}$ & $\begin{array}{l}\text { Dis- } \\
\text { solved } \\
\text { oxygen } \\
\text { (mg/L) }\end{array}$ & $\begin{array}{c}\text { Nitrite, } \\
\text { dis- } \\
\text { solved } \\
\text { (mg/Las } \\
\mathrm{N} \text { ) }\end{array}$ & $\begin{array}{c}\text { Nitrite } \\
\text { plus } \\
\text { nitrate, } \\
\text { dissolved } \\
\text { (mg/L as } \\
\mathrm{N})\end{array}$ & $\begin{array}{l}\text { Ammo- } \\
\text { nium, } \\
\text { dis- } \\
\text { solved } \\
\text { (mg/Las } \\
\mathrm{N})\end{array}$ & $\begin{array}{l}\text { Phospho- } \\
\text { rus ortho, } \\
\text { dissolved } \\
\text { (mg/L as } \\
\text { P) }\end{array}$ \\
\hline \multicolumn{11}{|l|}{ Illinois } \\
\hline IL04 & $08-12$ & - & 17.5 & 680 & 6.9 & 0.2 & $<0.01$ & $<0.05$ & 0.66 & 0.15 \\
\hline ILOS & $07-30$ & - & 17.0 & 685 & 6.9 & 1.6 & $<.01$ & 3.3 & .04 & .01 \\
\hline IL09 & $08-20$ & - & 12.5 & 652 & 7.6 & 2.0 & .01 & 27 & $<.01$ & $<.01$ \\
\hline IL12 & $08-11$ & - & 14.5 & 747 & 7.2 & .0 & $<.01$ & $<.05$ & .70 & .04 \\
\hline IL13 & $08-04$ & - & 17.0 & 1080 & 6.8 & 2.0 & $<.01$ & 7.2 & $<.01$ & $<.01$ \\
\hline IL18 & $07-29$ & - & 14.0 & 840 & 6.9 & .6 & $<.01$ & $<.05$ & .47 & .03 \\
\hline IL24 & $08-03$ & 40 & 14.5 & 786 & 7.3 & .0 & $<.01$ & $<.05$ & 3.9 & .22 \\
\hline IL25 & $08-05$ & - & 19.0 & 719 & 6.9 & 4.1 & $<.01$ & 2.4 & $<.01$ & .13 \\
\hline IL26 & $08-04$ & - & 17.5 & 293 & 7.7 & 1.9 & $<.01$ & 4.3 & $<.01$ & .05 \\
\hline IL27 & $08-05$ & - & 15.0 & 1260 & 7.1 & 3.0 & $<.01$ & 3.7 & $<.01$ & $<.01$ \\
\hline IL34 & $08-10$ & - & 13.5 & 499 & 7.1 & .1 & .01 & .69 & .14 & .06 \\
\hline IL36 & $08-20$ & - & 14.5 & 771 & 8.9 & 1.7 & $<.01$ & .31 & $<.01$ & $<.01$ \\
\hline IL39 & 08-06 & - & 16.5 & 720 & 7.1 & .1 & $<.01$ & $<.05$ & .51 & .01 \\
\hline IL40 & $07-31$ & - & 15.5 & 1420 & 8.9 & .0 & $<.01$ & $<.05$ & .16 & .08 \\
\hline IL41 & $07-29$ & - & 16.0 & 932 & 6.5 & .0 & $<.01$ & $<.05$ & .36 & .05 \\
\hline IL42 & $07-30$ & - & 16.0 & 733 & 7.3 & .0 & $<.01$ & $<.05$ & .29 & .01 \\
\hline IL44 & $07-31$ & - & 14.5 & 222 & 8.6 & .1 & $<.01$ & $<.05$ & .20 & .07 \\
\hline IL48 & $07-30$ & - & 12.0 & 530 & 6.9 & .2 & $<.01$ & $<.05$ & 1.0 & .02 \\
\hline IL53 & $07-30$ & - & 16.0 & 902 & 7.0 & 4.9 & $<.01$ & 7.7 & .02 & $<.01$ \\
\hline IL59 & $08-03$ & - & 16.0 & 692 & 6.6 & 3.8 & $<.01$ & 1.5 & .03 & .02 \\
\hline IL68 & $08-21$ & - & 16.0 & 554 & 7.1 & .1 & $<.01$ & $<.05$ & $<.01$ & $<.01$ \\
\hline \multicolumn{11}{|l|}{ Indiana } \\
\hline IN01 & $08-14$ & - & 12.5 & 801 & 7.0 & .2 & $<.01$ & $<.05$ & .10 & .01 \\
\hline IN04 & $08-26$ & - & 14.0 & 333 & 7.4 & .1 & $<.01$ & $<.05$ & 0.24 & $<.01$ \\
\hline IN06 & $08-25$ & - & 11.5 & 333 & 8.2 & 5.2 & $<.01$ & 6.6 & $<.01$ & .01 \\
\hline IN08 & 08-19 & 37 & 15.0 & 248 & 7.4 & 1.9 & $<.01$ & 2.4 & .02 & .01 \\
\hline
\end{tabular}


Table 2. Water-quality data from field measurements and laboratory analyses of water samples for inorganic nutrients, 1992-Continued

\begin{tabular}{|c|c|c|c|c|c|c|c|c|c|c|}
\hline $\begin{array}{c}\text { Site } \\
\text { identifier } \\
\text { (figs. 1-2) }\end{array}$ & $\begin{array}{c}\text { Date } \\
\text { sampled } \\
\text { (month, } \\
\text { day) }\end{array}$ & $\begin{array}{l}\text { Water } \\
\text { level } \\
\text { (feet) }\end{array}$ & $\begin{array}{c}\text { Water } \\
\text { temp- } \\
\text { perature } \\
\left({ }^{\circ} \mathrm{C}\right)\end{array}$ & $\begin{array}{c}\text { Specific } \\
\text { conduct- } \\
\text { ance } \\
(\mu \mathrm{S} / \mathrm{cm})\end{array}$ & $\begin{array}{c}\text { pH } \\
\text { (stan- } \\
\text { dard } \\
\text { units) }\end{array}$ & $\begin{array}{c}\text { Dis- } \\
\text { solved } \\
\text { oxygen } \\
\text { (mg/L) }\end{array}$ & $\begin{array}{c}\text { Nitrite, } \\
\text { dis- } \\
\text { solved } \\
\text { (mg/Las } \\
\mathrm{N})\end{array}$ & $\begin{array}{c}\text { Nitrite } \\
\text { plus } \\
\text { nitrate, } \\
\text { dissolved } \\
\text { (mg/L as } \\
\mathrm{N} \text { ) }\end{array}$ & $\begin{array}{l}\text { Ammo- } \\
\text { nium, } \\
\text { dis- } \\
\text { solved } \\
\text { (mg/Las } \\
\mathrm{N} \text { ) }\end{array}$ & $\begin{array}{l}\text { Phosp } \\
\text { rus ort } \\
\text { dissol } \\
\text { (mg/L } \\
\text { P) }\end{array}$ \\
\hline \multicolumn{11}{|c|}{ Indiana (Continued) } \\
\hline IN11 & $08-25$ & 17 & 12.5 & 233 & 7.6 & .1 & $<.01$ & $<.05$ & .35 & .03 \\
\hline IN12 & $08-20$ & - & 13.0 & 388 & 7.1 & .3 & $<.01$ & $<.05$ & .23 & $<.01$ \\
\hline IN14 & $08-24$ & - & 12.5 & 367 & 7.2 & 1.6 & $<.01$ & 6.3 & .02 & $<.01$ \\
\hline IN17 & $08-20$ & - & 13.0 & 712 & 7.3 & .1 & $<.01$ & $<.05$ & .43 & .02 \\
\hline IN23 & $08-21$ & - & 13.0 & 219 & 7.7 & .1 & $<.01$ & $<.05$ & .06 & $<.01$ \\
\hline IN28 & $08-26$ & - & 14.5 & 117 & 7.9 & 3.2 & .01 & 4.4 & .02 & $<.01$ \\
\hline IN29 & $08-17$ & - & 13.5 & 349 & 7.4 & 8.1 & $<.01$ & 8.3 & .01 & $<.01$ \\
\hline
\end{tabular}

lowa

$\begin{array}{lcccccccccc}\text { IA02 } & 08-14 & - & 14.0 & 633 & 6.4 & .6 & <.01 & <.05 & 1.4 & <.01 \\ \text { IA03 } & 08-20 & - & 14.5 & 581 & 7.3 & 11.8 & <.01 & 5.60 & <.01 & 0.03 \\ \text { IA05 } & 08-14 & 15 & 13.0 & 693 & 6.8 & 1.8 & .02 & 4.2 & .05 & <.01 \\ \text { IA08 } & 08-25 & - & 12.5 & 758 & 6.9 & .7 & <.01 & <.05 & .46 & <.01 \\ \text { IA12 } & 08-26 & - & 14.0 & 611 & 7.0 & .0 & <.01 & <.05 & .08 & <.01 \\ \text { IA13 } & 08-25 & 6 & 10.0 & 726 & 7.7 & .9 & <.01 & <.05 & .14 & <.01 \\ \text { IA15 } & 08-27 & 19.2 & 12.0 & 814 & 7.1 & 1.9 & <.01 & 17.0 & .03 & .02 \\ \text { IA18 } & 08-26 & - & 13.0 & 375 & 6.3 & .1 & .01 & <.05 & .51 & <.01 \\ \text { IA19 } & 08-13 & - & 11.0 & 570 & 7.6 & .7 & <.01 & 5.4 & <.01 & <.01 \\ \text { IA21 } & 08-26 & 9.71 & 13.0 & 382 & 6.3 & 9.2 & <.01 & 5.5 & <.01 & .06 \\ \text { IA24 } & 08-27 & 13.54 & 10.0 & 464 & 7.0 & 9.3 & <.01 & .94 & .42 & .13 \\ \text { IA25 } & 08-26 & 8.60 & 11.0 & 800 & 7.2 & .4 & <.01 & .92 & .17 & .02 \\ \text { IA26 } & 08-27 & - & 11.0 & 645 & 6.7 & 4.3 & <.01 & 6.0 & .02 & .16 \\ \text { IA27 } & 08-27 & - & 11.0 & 460 & 6.5 & 8.1 & <.01 & 12 & .01 & .10 \\ \text { IA28 } & 08-26 & 23 & 11.5 & 769 & 7.0 & 1.3 & <.01 & 6.9 & <.01 & .11 \\ \text { IA32 } & 08-13 & - & 13.0 & 498 & 6.2 & .2 & <.01 & <.05 & 1.3 & <.01 \\ \text { IA34 } & 08-26 & - & 10.5 & 590 & 7.2 & .4 & <.01 & 1.4 & .04 & .01\end{array}$


Table 2. Water-quality data from field measurements and laboratory analyses of water samples for inorganic nutrients, 1992-Continued

\begin{tabular}{|c|c|c|c|c|c|c|c|c|c|c|}
\hline $\begin{array}{c}\text { Site } \\
\text { identifier } \\
\text { (figs. 1-2) }\end{array}$ & $\begin{array}{c}\text { Date } \\
\text { sampled } \\
\text { (month, } \\
\text { day) }\end{array}$ & $\begin{array}{l}\text { Water } \\
\text { level } \\
\text { (feet) }\end{array}$ & $\begin{array}{c}\text { Water } \\
\text { temp- } \\
\text { perature } \\
\left({ }^{\circ} \mathrm{C}\right)\end{array}$ & $\begin{array}{c}\text { Specific } \\
\text { conduct- } \\
\text { ance } \\
(\mu \mathrm{S} / \mathrm{cm})\end{array}$ & $\begin{array}{c}\mathrm{pH} \\
\text { (stan- } \\
\text { dard } \\
\text { units) }\end{array}$ & $\begin{array}{c}\text { Dis- } \\
\text { solved } \\
\text { oxygen } \\
\text { (mg/L) }\end{array}$ & $\begin{array}{c}\text { Nitrite, } \\
\text { dis- } \\
\text { solved } \\
\text { (mg/Las } \\
\mathrm{N} \text { ) }\end{array}$ & $\begin{array}{c}\text { Nitrite } \\
\text { plus } \\
\text { nitrate, } \\
\text { dissolved } \\
(\mathrm{mg} / \mathrm{L} \text { as } \\
\mathrm{N})\end{array}$ & $\begin{array}{l}\text { Ammo- } \\
\text { nium, } \\
\text { dis- } \\
\text { solved } \\
\text { (mg/Las } \\
\mathrm{N} \text { ) }\end{array}$ & $\begin{array}{c}\text { Phosph } \\
\text { rus orth } \\
\text { dissolv } \\
\text { (mg/L } \\
\text { P) }\end{array}$ \\
\hline \multicolumn{11}{|l|}{ Michigan } \\
\hline M102 & $08-31$ & - & 11.5 & 864 & 7.3 & .1 & $<.01$ & .07 & .15 & $<.01$ \\
\hline MI05 & $08-27$ & - & 11.5 & 665 & 7.5 & .0 & $<.01$ & $<.05$ & .01 & $<.01$ \\
\hline M113 & $08-28$ & - & 16.0 & 537 & 7.5 & .0 & $<.01$ & .08 & .01 & .01 \\
\hline MI14 & $08-28$ & - & 12.0 & 1110 & 7.2 & .2 & $<.01$ & $<.05$ & .05 & $<.01$ \\
\hline \multicolumn{11}{|l|}{ Minnesota } \\
\hline MN02 & $08-17$ & - & 13.5 & 568 & 7.4 & 16 & $<.01$ & 2.5 & $<.01$ & .02 \\
\hline MN04 & $08-18$ & - & 11.5 & 2700 & 6.8 & .1 & $<.01$ & .07 & 2.0 & $<.01$ \\
\hline MN06 & $08-18$ & - & 10.5 & 913 & 7.5 & .1 & $<.01$ & .12 & 5.7 & .34 \\
\hline MN12 & $08-18$ & - & 19.0 & 2710 & 7.5 & 11 & $<.01$ & .08 & 1.8 & .02 \\
\hline MN14 & $08-14$ & - & 11.0 & 1170 & 7.7 & 6.6 & $<.01$ & 3.5 & .02 & .07 \\
\hline MN15 & $08-18$ & - & 10.5 & 1100 & 7.1 & 3.6 & $<.01$ & 21 & .02 & .04 \\
\hline MN16 & $08-18$ & - & 10.5 & 1920 & 6.6 & .2 & $<.01$ & .07 & .12 & $<.01$ \\
\hline MN21 & $08-17$ & - & 14.5 & 516 & 7.7 & .1 & $<.01$ & .08 & .03 & .01 \\
\hline MN24 & 08-17 & - & 11.0 & 502 & 7.7 & .1 & $<.01$ & .08 & .02 & $<.01$ \\
\hline
\end{tabular}

Missouri

$\begin{array}{llllrrrrrrr}\text { MO06 } & 08-12 & - & 14.5 & 765 & 7.1 & .2 & <.01 & <.05 & .26 & .15 \\ \text { MO10 } & 08-12 & - & 15.0 & 1270 & 7.1 & .9 & <.01 & .70 & 1.4 & .18 \\ \text { MO11 } & 08-10 & 41.0 & 15.5 & 659 & 7.2 & 6.0 & .02 & 1.9 & .02 & .03 \\ \text { MO16 } & 08-11 & - & 16.0 & 648 & 7.3 & .1 & <.01 & <.05 & .18 & .01 \\ \text { MO21 } & 08-13 & - & 18.0 & 2360 & 7.8 & .1 & <.01 & <.05 & .27 & <.01\end{array}$

Nebraska

$\begin{array}{lllllllllll}\text { NE05 } & 08-04 & - & 14.0 & 2000 & 7.0 & 2.0 & <.01 & 3.9 & .03 & .19 \\ \text { NE15 } & 08-04 & - & 20.0 & 960 & 7.3 & 7.6 & <.01 & 20 & .05 & .02 \\ \text { NE17 } & 08-07 & 48 & 13.5 & 740 & 7.1 & 5.6 & <.01 & 3.6 & .01 & .09\end{array}$


Table 2. Water-quality data from field measurements and laboratory analyses of water samples for inorganic nutrients, 1992-Continued

\begin{tabular}{|c|c|c|c|c|c|c|c|c|c|c|}
\hline $\begin{array}{c}\text { Site } \\
\text { identifier } \\
\text { (figs. 1-2) }\end{array}$ & $\begin{array}{c}\text { Date } \\
\text { sampled } \\
\text { (month, } \\
\text { day) }\end{array}$ & $\begin{array}{l}\text { Water } \\
\text { level } \\
\text { (feet) }\end{array}$ & $\begin{array}{c}\text { Water } \\
\text { temp- } \\
\text { perature } \\
\left({ }^{\circ} \mathrm{C}\right)\end{array}$ & $\begin{array}{c}\text { Specific } \\
\text { conduct- } \\
\text { ance } \\
(\mu \mathrm{S} / \mathrm{cm})\end{array}$ & $\begin{array}{l}\text { pH } \\
\text { (stan- } \\
\text { dard } \\
\text { units) }\end{array}$ & $\begin{array}{c}\text { Dis- } \\
\text { solved } \\
\text { oxygen } \\
\text { (mg/L) }\end{array}$ & $\begin{array}{c}\text { Nitrite, } \\
\text { dis- } \\
\text { solved } \\
\text { (mg/Las } \\
\mathrm{N})\end{array}$ & $\begin{array}{c}\text { Nitrite } \\
\text { plus } \\
\text { nitrate, } \\
\text { dissolved } \\
\text { (mg/L as } \\
\mathrm{N})\end{array}$ & $\begin{array}{c}\text { Ammo- } \\
\text { nium, } \\
\text { dis- } \\
\text { solved } \\
\text { (mg/Las } \\
\mathrm{N})\end{array}$ & $\begin{array}{l}\text { Phospho- } \\
\text { rus ortho, } \\
\text { dissolved } \\
\text { (mg/L as } \\
\text { P) }\end{array}$ \\
\hline \multicolumn{11}{|c|}{ Nebraska (Continued) } \\
\hline NE18 & 08-07 & - & 12.5 & 842 & 7.0 & 1.7 & $<.01$ & .91 & .06 & .20 \\
\hline NE19 & $08-04$ & 21 & 12.5 & 744 & 7.0 & 8.6 & $<.01$ & 6.7 & .04 & .24 \\
\hline NE21 & $08-03$ & - & 13.5 & 501 & 7.1 & 3.1 & $<.01$ & 1.7 & .02 & .18 \\
\hline NE24 & $08-05$ & - & 12.5 & 356 & 7.7 & 7.0 & $<.01$ & .37 & .03 & $<.01$ \\
\hline NE25 & $08-03$ & - & 12.0 & 682 & 7.2 & .2 & $<.01$ & .37 & .02 & .28 \\
\hline NE27 & $08-05$ & - & 12.5 & 972 & 7.6 & .2 & .01 & .17 & .05 & .02 \\
\hline NE28 & $08-03$ & - & 12.0 & 608 & 7.1 & 5.7 & $<.01$ & 4.3 & .06 & .21 \\
\hline NE35 & $08-05$ & - & 14.5 & 1040 & 7.2 & 2.1 & $<.01$ & 5.0 & .02 & .03 \\
\hline NE36 & $08-06$ & - & 13.0 & 559 & 6.7 & 1.9 & $<.01$ & 7.1 & .01 & .45 \\
\hline NE39 & 08-04 & - & 14.5 & 90 & 6.9 & 4.5 & $<.01$ & .25 & .05 & .35 \\
\hline NE40 & 08-03 & - & 13.0 & 298 & 6.6 & 8.0 & $<.01$ & 2.2 & .06 & .20 \\
\hline NE41 & 08-03 & - & 13.5 & 745 & 6.9 & .4 & .04 & 1.1 & .22 & .22 \\
\hline NE42 & 08-05 & - & 13.3 & 716 & 7.2 & 2.9 & $<.01$ & 2.6 & .02 & .07 \\
\hline
\end{tabular}

Ohio

$\begin{array}{lllllllllrr}\text { OH07 } & 08-19 & - & 13.0 & 699 & 7.0 & .1 & <.01 & <.05 & .33 & <.01 \\ \text { OH13 } & 08-18 & - & 11.5 & 585 & 7.4 & .2 & <.01 & <.05 & .12 & <.01 \\ \text { OH16 } & 08-17 & 33.34 & 14.5 & 795 & 7.3 & .4 & <.01 & <.05 & .25 & .02 \\ \text { OH18 } & 08-18 & 10.95 & 16.0 & 710 & 7.3 & 2.9 & <.01 & <.05 & .11 & <.01 \\ \text { OH19 } & 08-19 & - & 13.0 & 795 & 7.4 & .1 & <.01 & <.05 & .25 & .02 \\ \text { OH25 } & 08-18 & - & 13.0 & 2550 & 7.7 & .2 & <.01 & <.05 & .60 & <.01 \\ \text { OH26 } & 08-18 & - & 12.5 & 980 & 7.2 & .4 & <.01 & <.05 & .28 & .01 \\ \text { OH27 } & 08-18 & - & 12.5 & 710 & 7.4 & .2 & <.01 & <.05 & .10 & <.01 \\ \text { OH30 } & 08-17 & - & 12.0 & 950 & 7.4 & .1 & <.01 & <.05 & .49 & <.01\end{array}$


Table 2. Water-quality data from field measurements and laboratory analyses of water samples for inorganic nutrients, 1992-Continued

\begin{tabular}{|c|c|c|c|c|c|c|c|c|c|c|}
\hline $\begin{array}{c}\text { Site } \\
\text { identifier } \\
\text { (figs. 1-2) }\end{array}$ & $\begin{array}{c}\text { Date } \\
\text { sampled } \\
\text { (month, } \\
\text { day) }\end{array}$ & $\begin{array}{l}\text { Water } \\
\text { level } \\
\text { (feet) }\end{array}$ & $\begin{array}{l}\text { Water } \\
\text { temp- } \\
\text { perature } \\
\left({ }^{\circ} \mathrm{C}\right)\end{array}$ & $\begin{array}{c}\text { Specific } \\
\text { conduct- } \\
\text { ance } \\
(\mu \mathrm{S} / \mathrm{cm})\end{array}$ & $\begin{array}{c}\mathrm{pH} \\
\text { (stan- } \\
\text { dard } \\
\text { units) }\end{array}$ & $\begin{array}{c}\text { Dis- } \\
\text { solved } \\
\text { oxygen } \\
\text { (mg/L) }\end{array}$ & $\begin{array}{l}\text { Nitrite, } \\
\text { dis- } \\
\text { solved } \\
\text { (mg/Las } \\
\mathrm{N})\end{array}$ & $\begin{array}{c}\text { Nitrite } \\
\text { plus } \\
\text { nitrate, } \\
\text { dissolved } \\
\text { (mg/L as } \\
\mathrm{N})\end{array}$ & $\begin{array}{l}\text { Ammo- } \\
\text { nium, } \\
\text { dis- } \\
\text { solved } \\
\text { (mg/Las } \\
\mathrm{N})\end{array}$ & $\begin{array}{l}\text { Phospho- } \\
\text { rus ortho, } \\
\text { dissolved } \\
\text { (mg/L as } \\
\text { P) }\end{array}$ \\
\hline \multicolumn{11}{|l|}{ Wisconsin } \\
\hline WI02 & $08-20$ & - & 11.5 & 198 & 7.2 & 7.5 & $<.01$ & 8.8 & .03 & .10 \\
\hline WI03 & $08-20$ & - & 10.5 & 268 & 6.8 & 4.2 & $<.01$ & 14 & .03 & $<.01$ \\
\hline WI05 & $08-19$ & - & 10.5 & 615 & 7.5 & 8.2 & $<.01$ & 5.1 & .02 & .01 \\
\hline WI06 & $08-25$ & - & 11.5 & 662 & 7.2 & 9.5 & $<.01$ & 12 & .03 & $<.01$ \\
\hline WI07 & $08-20$ & - & 12.0 & 418 & 6.5 & 7.2 & $<.01$ & 17 & .02 & $<.01$ \\
\hline WI10 & $08-19$ & - & 11.0 & 536 & 7.3 & 1.0 & $<.01$ & $<.05$ & .06 & $<.01$ \\
\hline WI11 & $08-18$ & - & 10.5 & 619 & 7.5 & 5.2 & $<.01$ & 7.6 & .03 & $<.01$ \\
\hline WI13 & $08-18$ & - & 10.5 & 930 & 7.5 & 1.2 & $<.01$ & $<.05$ & .06 & $<.01$ \\
\hline WI17 & $08-19$ & - & 11.5 & 628 & 7.2 & 5.5 & .01 & .38 & .03 & $<.01$ \\
\hline
\end{tabular}


Table 3. Water-quality data from routine laboratory analyses of water samples for selected herbicides and herbicide metabolites, 1992

[Samples were analyzed for the compounds ametryn, deethylcyanazine amide, deethylcyanazine, prometryn, propazine, and terbutryn, but these compounds were not detected above $0.05 \mu \mathrm{g} / \mathrm{L}$ reporting limits. $\mu \mathrm{g} / \mathrm{L}$, micrograms per liter; Alachlor-ESA, alachlor ethanesulfonic acid; <, less than]

\begin{tabular}{|c|c|c|c|c|c|c|c|c|c|c|c|}
\hline $\begin{array}{c}\text { Site } \\
\text { identifier } \\
\text { (figs. 1-2) }\end{array}$ & $\begin{array}{l}\text { Ala- } \\
\text { chlor } \\
(\mu g / L)\end{array}$ & $\begin{array}{l}\text { Atra- } \\
\text { zine } \\
(\mu g / L)\end{array}$ & $\begin{array}{c}\text { Cyana- } \\
\text { zine } \\
(\mu g / L)\end{array}$ & $\begin{array}{l}\text { Cyana- } \\
\text { zine } \\
\text { Amide } \\
(\mu g / L)\end{array}$ & $\begin{array}{c}\text { Deiso- } \\
\text { propyl- } \\
\text { atrazine } \\
(\mu g / L)\end{array}$ & $\begin{array}{c}\text { Deethyl } \\
\text {-atra- } \\
\text { zine } \\
(\mu g / L)\end{array}$ & $\begin{array}{l}\text { Ala- } \\
\text { chlor- } \\
\text { ESA } \\
(\mu \mathrm{g} / \mathrm{L})\end{array}$ & $\begin{array}{l}\text { Meto- } \\
\text { lachlor } \\
(\mu \mathrm{g} / \mathrm{L})\end{array}$ & $\begin{array}{l}\text { Metri- } \\
\text { buzin } \\
(\mu g / L)\end{array}$ & $\begin{array}{l}\text { Pro- } \\
\text { meton } \\
(\mu g / L)\end{array}$ & $\begin{array}{c}\text { Sim- } \\
\text { azine } \\
(\mu g / L)\end{array}$ \\
\hline \multicolumn{12}{|l|}{ Illinois } \\
\hline IL04 & $<0.05$ & $<0.05$ & $<0.05$ & $<0.05$ & $<0.05$ & $<0.05$ & 1.0 & $<0.05$ & $<0.05$ & $<0.05$ & $<0.05$ \\
\hline IL05 & $<.05$ & $<.05$ & $<.05$ & $<.05$ & $<.05$ & $<.05$ & $<.10$ & $<.05$ & $<.05$ & $<.05$ & $<.05$ \\
\hline IL09 & $<.05$ & $<.05$ & $<.05$ & $<.05$ & $<.05$ & $<.05$ & .78 & $<.05$ & $<.05$ & $<.05$ & $<.05$ \\
\hline IL13 & $<.05$ & $<.05$ & $<.05$ & $<.05$ & $<.05$ & $<.05$ & .53 & $<.05$ & $<.05$ & $<.05$ & $<.05$ \\
\hline IL18 & $<.05$ & .26 & $<.05$ & $<.05$ & .05 & .21 & .13 & $<.05$ & $<.05$ & .08 & $<.05$ \\
\hline IL25 & $<.05$ & .08 & $<.05$ & .06 & .28 & .15 & $<.10$ & $<.05$ & $<.05$ & $<.05$ & $<.05$ \\
\hline IL26 & $<.05$ & $<.05$ & $<.05$ & $<.05$ & $<.05$ & $<.05$ & $<.10$ & $<.05$ & $<.05$ & $<.05$ & $<.05$ \\
\hline IL27 & $<.05$ & $<.05$ & $<.05$ & $<.05$ & $<.05$ & $<.05$ & $<.10$ & $<.05$ & $<.05$ & $<.05$ & $<.05$ \\
\hline IL34 & $<.05$ & $<.05$ & $<.05$ & $<.05$ & $<.05$ & $<.05$ & .77 & $<.05$ & $<.05$ & $<.05$ & $<.05$ \\
\hline IL39 & $<.05$ & .45 & $<.05$ & $<.05$ & $<.05$ & .05 & .94 & $<.05$ & $<.05$ & $<.05$ & $<.05$ \\
\hline IL41 & $<.05$ & .07 & $<.05$ & $<.05$ & $<.05$ & $<.05$ & .15 & $<.05$ & $<.05$ & $<.05$ & $<.05$ \\
\hline IL42 & $<.05$ & $<.05$ & $<.05$ & $<.05$ & $<.05$ & $<.05$ & $<.10$ & $<.05$ & $<.05$ & $<.05$ & $<.05$ \\
\hline IL48 & $<.05$ & $<.05$ & $<.05$ & $<.05$ & $<.05$ & $<.05$ & $<.10$ & $<.05$ & $<.05$ & $<.05$ & $<.05$ \\
\hline IL53 & $<.05$ & .22 & $<.05$ & $<.05$ & .05 & .20 & .22 & $<.05$ & $<.05$ & .08 & $<.05$ \\
\hline IL59 & $<.05$ & $<.05$ & $<.05$ & $<.05$ & $<.05$ & $<.05$ & $<.10$ & $<.05$ & $<.05$ & $<.05$ & $<.05$ \\
\hline
\end{tabular}

Indiana

$\begin{array}{llllllllllll}\text { IN01 } & <.05 & <.05 & <.05 & <.05 & <.05 & <.05 & .53 & <.05 & <.05 & <.05 & <.05 \\ \text { IN04 } & <.05 & <.05 & <.05 & <.05 & <.05 & <.05 & 5.0 & <.05 & <.05 & <.05 & <.05 \\ \text { IN06 } & <.05 & <.05 & <.05 & <.05 & <.05 & <.05 & .63 & <.05 & <.05 & <.05 & <.05 \\ \text { IN08 } & <.05 & <.05 & <.05 & <.05 & <.05 & <.05 & <.10 & <.05 & <.05 & <.05 & <.05 \\ \text { IN14 } & <.05 & .12 & <.05 & <.05 & <.05 & .09 & .36 & <.05 & <.05 & <.05 & <.05 \\ \text { IN28 } & <.05 & <.05 & <.05 & <.05 & <.05 & <.05 & 1.3 & <.05 & <.05 & <.05 & <.05 \\ \text { IN29 } & <.05 & .18 & <.05 & .11 & .23 & .35 & <.10 & <.05 & <.05 & <.05 & <.05\end{array}$


Table 3. Water-quality data from routine laboratory analyses of water samples for selected herbicides and herbicide metabolites, 1992-Continued

\begin{tabular}{|c|c|c|c|c|c|c|c|c|c|c|c|}
\hline $\begin{array}{c}\text { Site } \\
\text { identifier } \\
\text { (figs. 1-2) }\end{array}$ & $\begin{array}{l}\text { Ala- } \\
\text { chlor } \\
(\mu g / L)\end{array}$ & $\begin{array}{c}\text { Atra- } \\
\text { zine } \\
(\mu g / L)\end{array}$ & $\begin{array}{c}\text { Cyana- } \\
\text { zine } \\
(\mu g / L)\end{array}$ & $\begin{array}{c}\text { Cyana- } \\
\text { zine } \\
\text { Amide } \\
(\mu g / L)\end{array}$ & $\begin{array}{c}\text { Deiso- } \\
\text { propyl- } \\
\text { atrazine } \\
(\mu g / L)\end{array}$ & $\begin{array}{c}\text { Deethyl } \\
\text {-atra- } \\
\text { zine } \\
(\mu g / L)\end{array}$ & $\begin{array}{l}\text { Ala- } \\
\text { chlor- } \\
\text { ESA } \\
(\mu g / L)\end{array}$ & $\begin{array}{l}\text { Meto- } \\
\text { lachlor } \\
(\mu g / L)\end{array}$ & $\begin{array}{l}\text { Metri- } \\
\text { buzin } \\
(\mu g / L)\end{array}$ & $\begin{array}{l}\text { Pro- } \\
\text { meton } \\
(\mu g / L)\end{array}$ & $\begin{array}{l}\text { Sim- } \\
\text { azine } \\
(\mu g / L)\end{array}$ \\
\hline \multicolumn{12}{|l|}{ lowa } \\
\hline IA03 & $<.05$ & .06 & $<.05$ & $<.05$ & .07 & .10 & .07 & $<.05$ & $<.05$ & $<.05$ & $<.05$ \\
\hline IA05 & .25 & .98 & $<.05$ & .19 & .12 & .18 & .93 & .21 & .05 & .10 & $<.05$ \\
\hline IA 12 & $<.05$ & $<.05$ & $<.05$ & $<.05$ & $<.05$ & $<.05$ & $<.10$ & $<.05$ & $<.05$ & $<.05$ & $<.05$ \\
\hline IA13 & $<.05$ & $<.05$ & $<.05$ & $<.05$ & $<.05$ & $<.05$ & .43 & $<.05$ & $<.05$ & $<.05$ & $<.05$ \\
\hline IA15 & .11 & 1.0 & $<.05$ & .55 & .27 & .40 & 2.5 & .76 & $<.05$ & $<.05$ & $<.05$ \\
\hline IA18 & $<.05$ & $<.05$ & $<.05$ & $<.05$ & $<.05$ & $<.05$ & .06 & $<.05$ & $<.05$ & $<.05$ & $<.05$ \\
\hline IA19 & $<05$ & .05 & $<.05$ & $<.05$ & $<.05$ & .09 & .72 & $<.05$ & $<.05$ & $<.05$ & $<.05$ \\
\hline IA21 & $<.05$ & $<.05$ & $<.05$ & $<.05$ & $<.05$ & $<.05$ & .05 & $<.05$ & $<.05$ & $<.05$ & $<.05$ \\
\hline IA 24 & $<.05$ & $<.05$ & $<.05$ & $<.05$ & $<.05$ & $<.05$ & $<.10$ & $<.05$ & $<.05$ & $<.05$ & $<.05$ \\
\hline IA25 & $<.05$ & $<.05$ & $<.05$ & $<.05$ & $<.05$ & $<.05$ & .62 & $<.05$ & $<.05$ & .08 & $<.05$ \\
\hline IA26 & $<.05$ & $<.05$ & $<.05$ & $<.05$ & $<.05$ & $<.05$ & .40 & $<.05$ & $<.05$ & $<.05$ & $<.05$ \\
\hline IA 28 & $<.05$ & $<.05$ & $<.05$ & $<.05$ & $<.05$ & $<.05$ & $<.10$ & $<.05$ & $<.05$ & $<.05$ & $<.05$ \\
\hline IA34 & $<.05$ & $<.05$ & $<.05$ & $<.05$ & $<.05$ & $<.05$ & .70 & $<.05$ & $<.05$ & $<.05$ & $<.05$ \\
\hline \multicolumn{12}{|l|}{ Michigan } \\
\hline MI13 & $<05$ & .17 & $<.05$ & $<.05$ & .06 & .09 & .82 & $<.05$ & $<.05$ & $<.05$ & $<.05$ \\
\hline \multicolumn{12}{|l|}{ Minnesota } \\
\hline MN02 & $<05$ & .06 & $<.05$ & $<.05$ & $<.05$ & .06 & $<.10$ & $<.05$ & $<.05$ & $<.05$ & $<.05$ \\
\hline MN14 & $<.05$ & $<.05$ & $<.05$ & $<.05$ & $<.05$ & .11 & $<.10$ & $<.05$ & $<.05$ & $<.05$ & $<.05$ \\
\hline MN16 & $<05$ & $<.05$ & $<.05$ & $<.05$ & $<.05$ & $<.05$ & .11 & $<.05$ & $<.05$ & $<.05$ & $<.05$ \\
\hline MN24 & $<.05$ & $<.05$ & $<.05$ & $<.05$ & $<.05$ & $<.05$ & $<.10$ & $<.05$ & $<.05$ & $<.05$ & $<.05$ \\
\hline \multicolumn{12}{|l|}{ Missouri } \\
\hline MO06 & .99 & $<.05$ & $<.05$ & $<.05$ & $<.05$ & $<.05$ & $<.10$ & .08 & $<.05$ & $<.05$ & $<.05$ \\
\hline MO11 & $<05$ & .12 & $<.05$ & .06 & $<.05$ & .06 & $<.10$ & $<.05$ & $<.05$ & $<.05$ & $<.05$ \\
\hline
\end{tabular}


Table 3. Water-quality data from routine laboratory analyses of water samples for selected herbicides and herbicide metabolites, 1992-Continued

\begin{tabular}{|c|c|c|c|c|c|c|c|c|c|c|c|}
\hline $\begin{array}{c}\text { Site } \\
\text { identifier } \\
\text { (figs. 1-2) }\end{array}$ & $\begin{array}{l}\text { Ala- } \\
\text { chlor } \\
(\mu g / L)\end{array}$ & $\begin{array}{l}\text { Atra- } \\
\text { zine } \\
(\mu g / L)\end{array}$ & $\begin{array}{c}\text { Cyana- } \\
\text { zine } \\
(\mu g / L)\end{array}$ & $\begin{array}{l}\text { Cyana- } \\
\text { zine } \\
\text { Amide } \\
(\mu g / L)\end{array}$ & $\begin{array}{c}\text { Deiso- } \\
\text { propyl- } \\
\text { atrazine } \\
(\mu \mathrm{g} / \mathrm{L})\end{array}$ & $\begin{array}{c}\text { Deethyl } \\
\text {-atra- } \\
\text { zine } \\
(\mu \mathrm{g} / \mathrm{L})\end{array}$ & $\begin{array}{c}\text { Ala- } \\
\text { chlor- } \\
\text { ESA } \\
(\mu \mathrm{g} / \mathrm{L})\end{array}$ & $\begin{array}{l}\text { Meto- } \\
\text { lachlor } \\
(\mu g / L)\end{array}$ & $\begin{array}{l}\text { Metri- } \\
\text { buzin } \\
(\mu g / L)\end{array}$ & $\begin{array}{l}\text { Pro- } \\
\text { meton } \\
(\mu g / L)\end{array}$ & $\begin{array}{l}\text { Sim- } \\
\text { azine } \\
(\mu g / L)\end{array}$ \\
\hline \multicolumn{12}{|l|}{ Nebraska } \\
\hline NE05 & $<.05$ & .19 & $<.05$ & $<.05$ & $<.05$ & .19 & $<.10$ & $<.05$ & $<.05$ & .47 & $<.05$ \\
\hline NE15 & $<.05$ & .08 & $<.05$ & $<.05$ & $<.05$ & $<.05$ & $<.10$ & $<.05$ & $<.05$ & $<.05$ & $<.05$ \\
\hline NE17 & $<.05$ & $<.05$ & $<.05$ & $<.05$ & $<.05$ & $<.05$ & $<.10$ & $<.05$ & $<.05$ & $<.05$ & $<.05$ \\
\hline NE19 & $<.05$ & 1.0 & $<.05$ & $<.05$ & .15 & .59 & $<.10$ & $<.05$ & $<.05$ & $<.05$ & $<.05$ \\
\hline NE24 & $<.05$ & $<.05$ & $<.05$ & $<.05$ & $<.05$ & $<.05$ & $<.10$ & $<.05$ & $<.05$ & $<.05$ & $<.05$ \\
\hline NE25 & $<.05$ & .05 & $<.05$ & $<.05$ & $<.05$ & $<.05$ & $<.10$ & $<.05$ & $<.05$ & .10 & $<.05$ \\
\hline NE27 & $<.05$ & .15 & $<.05$ & $<.05$ & $<.05$ & .06 & $<.10$ & $<.05$ & $<.05$ & $<.05$ & $<.05$ \\
\hline NE35 & $<.05$ & .50 & $<.05$ & $<.05$ & .20 & 1.8 & 1.6 & $<.05$ & $<.05$ & $<.05$ & $<.05$ \\
\hline NE36 & $<.05$ & .18 & $<.05$ & $<.05$ & $<.05$ & .12 & $<.10$ & $<.05$ & $<.05$ & $<.05$ & $<.05$ \\
\hline NE39 & $<.05$ & $<.05$ & $<.05$ & $<.05$ & $<.05$ & $<.05$ & $<.10$ & $<.05$ & $<.05$ & $<.05$ & $<.05$ \\
\hline NE40 & $<.05$ & $<.05$ & $<.05$ & $<.05$ & $<.05$ & $<.05$ & $<.10$ & $<.05$ & $<.05$ & $<.05$ & $<.05$ \\
\hline NE41 & $<.05$ & .49 & $<.05$ & $<.05$ & .05 & .15 & $<.10$ & $<.05$ & $<.05$ & 1.4 & .09 \\
\hline NE42 & $<.05$ & $<.05$ & $<.05$ & $<.05$ & $<.05$ & $<.05$ & $<.10$ & $<.05$ & $<.05$ & $<.05$ & $<.05$ \\
\hline
\end{tabular}

Ohio

$\begin{array}{llllllllllll}\mathrm{OH} 07 & <.05 & <.05 & <.05 & <.05 & <.05 & <.05 & .14 & <.05 & <.05 & <.05 & <.05 \\ \mathrm{OH} 18 & <.05 & <.05 & <.05 & <.05 & <.05 & <.05 & .22 & <.05 & <.05 & <.05 & <.05 \\ \mathrm{OH} 25 & <.05 & <.05 & <.05 & <.05 & <.05 & <.05 & <.10 & <.05 & <.05 & <.05 & <.05 \\ \mathrm{OH} 30 & <.05 & <.05 & <.05 & <.05 & <.05 & <.05 & <.10 & <.05 & <.05 & <.05 & <.05\end{array}$

Wisconsin

\begin{tabular}{llrrrrrrrrrr} 
W102 & $<.05$ & $<.05$ & $<.05$ & $<.05$ & $<.05$ & $<.05$ & .83 & $<.05$ & $<.05$ & $<.05$ & $<.05$ \\
W103 & $<.05$ & $<.05$ & $<.05$ & $<.05$ & $<.05$ & .05 & 1.0 & $<.05$ & $<.05$ & $<.05$ & $<.05$ \\
W105 & $<.05$ & $<.05$ & $<.05$ & $<.05$ & $<.05$ & .11 & .28 & $<.05$ & $<.05$ & $<.05$ & $<.05$ \\
W106 & $<.05$ & .05 & $<.05$ & $<.05$ & $<.05$ & .20 & 2.9 & $<.05$ & $<.05$ & $<.05$ & $<.05$ \\
W107 & $<.05$ & $<.05$ & $<.05$ & $<.05$ & $<.05$ & $<.05$ & 1.2 & $<.05$ & $<.05$ & $<.05$ & $<.05$ \\
W110 & $<.05$ & $<.05$ & $<.05$ & $<.05$ & $<.05$ & $<.05$ & $<.10$ & $<.05$ & $<.05$ & $<.05$ & $<.05$ \\
W111 & $<.05$ & .09 & $<.05$ & .09 & .06 & .22 & 1.0 & $<.05$ & $<.05$ & $<.05$ & $<.05$ \\
\hline
\end{tabular}


Table 4. Water-quality data from sensitive analytical methods of water samples for selected pesticides and pesticide metabolites, $1992^{1}$

[ $\mu \mathrm{g} / \mathrm{L}$, micrograms per liter; DEA, Deethylatrazine; <, less than]

\begin{tabular}{|c|c|c|c|c|c|c|c|c|c|c|}
\hline $\begin{array}{c}\text { Site } \\
\text { identifier } \\
\text { (figs. 1-2) }\end{array}$ & $\begin{array}{c}\text { Atrazine } \\
(\mu \mathrm{g} / \mathrm{L})\end{array}$ & $\begin{array}{c}\text { DEA } \\
(\mu \mathrm{g} / \mathrm{L})\end{array}$ & $\begin{array}{c}\text { 2,6-Die- } \\
\text { thani- } \\
\text { line }(\mu g / L)\end{array}$ & $\begin{array}{l}\text { Sima- } \\
\text { zine } \\
(\mu \mathrm{g} / \mathrm{L})\end{array}$ & $\begin{array}{l}\text { Metol- } \\
\text { achlor } \\
(\mu g / L)\end{array}$ & $\begin{array}{l}\text { Pro- } \\
\text { meton } \\
(\mu g / L)\end{array}$ & $\begin{array}{c}\text { P,P' } \\
\text { DDE } \\
(\mu \mathrm{g} / \mathrm{L})\end{array}$ & $\begin{array}{l}\text { Ala- } \\
\text { chlor } \\
(\mu \mathrm{g} / \mathrm{L})\end{array}$ & $\begin{array}{l}\text { Chlor- } \\
\text { pyrifos } \\
(\mu g / L)\end{array}$ & $\begin{array}{l}\text { Ethal- } \\
\text { fluralin } \\
(\mu \mathrm{g} / \mathrm{L})\end{array}$ \\
\hline \multicolumn{11}{|l|}{ Illinois } \\
\hline IL04 & $<0.005$ & $<0.003$ & 0.004 & $<0.005$ & 0.003 & $<0.01$ & $<0.008$ & $<0.003$ & $<0.002$ & $<0.005$ \\
\hline IL09 & $<.005$ & $<.003$ & .007 & $<.005$ & $<.002$ & $<.01$ & $<.008$ & $<.003$ & $<.002$ & $<.005$ \\
\hline IL13 & .029 & $<.003$ & .003 & .007 & $<.002$ & .01 & $<.008$ & $<.003$ & $<.002$ & $<.005$ \\
\hline IL24 & $<.005$ & $<.003$ & $<.002$ & $<.005$ & $<.002$ & $<.01$ & .009 & $<.003$ & $<.002$ & $<.005$ \\
\hline IL25 & .068 & .012 & $<.002$ & $<.005$ & .024 & .02 & $<.008$ & $<.003$ & $<.002$ & $<.005$ \\
\hline IL26 & $<.005$ & .004 & $<.002$ & $<.005$ & $<.002$ & $<.01$ & $<.008$ & $<.003$ & $<.002$ & $<.005$ \\
\hline IL34 & .022 & $<.003$ & .005 & $<.005$ & .011 & $<.01$ & $<.008$ & $<.003$ & $<.002$ & $<.005$ \\
\hline IL41 & .061 & $<.003$ & $<.002$ & $<.005$ & $<.002$ & $<.01$ & $<.008$ & $<.003$ & $<.002$ & $<.005$ \\
\hline
\end{tabular}

Indiana

$\begin{array}{lrrrrrrrrrr}\text { IN06 } & <.005 & .004 & .002 & <.005 & <.002 & <.01 & <.008 & <.003 & <.002 & <.005 \\ \text { IN14 } & .090 & .007 & .003 & <.005 & <.002 & <.01 & <.008 & <.003 & <.002 & <.005 \\ \text { IN17 } & <.005 & <.003 & .003 & <.005 & <.002 & <.01 & <.008 & <.003 & <.002 & <.005\end{array}$

lowa

$\begin{array}{lrrrrrrrrrr}\text { IA05 } & .560 & .012 & .007 & .010 & .180 & .05 & <.008 & .190 & <.002 & <.005 \\ \text { IA15 } & .580 & .036 & <.002 & <.005 & .570 & <.01 & <.008 & .079 & <.002 & <.005 \\ \text { IA18 } & <.005 & <.003 & .003 & <.005 & <.002 & <.01 & <.008 & <.003 & <.002 & <.005 \\ \text { IA19 } & .035 & .007 & .003 & <.005 & <.002 & <.01 & <.008 & <.003 & <.002 & <.005 \\ \text { IA21 } & .010 & .005 & <.002 & <.005 & <.002 & <.01 & <.008 & <.003 & <.002 & <.005 \\ \text { IA25 } & .010 & <.003 & <.002 & <.005 & .019 & <.01 & <.008 & <.003 & <.002 & <.005 \\ \text { IA26 } & .016 & <.003 & .002 & <.005 & <.002 & <.01 & <.008 & <.003 & <.002 & <.005 \\ \text { IA28 } & .011 & <.003 & <.002 & <.005 & <.002 & <.01 & <.008 & <.003 & <.002 & <.005 \\ \text { IA32 } & .009 & <.003 & <.002 & <.005 & <.002 & <.01 & <.008 & <.003 & <.002 & <.005 \\ \text { IA34 } & <.005 & <.003 & .006 & <.005 & <.002 & <.01 & <.008 & <.003 & <.002 & <.005\end{array}$


Table 4. Water-quality data from sensitive analytical methods of water samples for selected pesticides and pesticide metabolites, 1992-Continued

\begin{tabular}{|c|c|c|c|c|c|c|c|c|c|c|}
\hline $\begin{array}{c}\text { Site } \\
\text { identifier } \\
\text { (figs. 1-2) }\end{array}$ & $\begin{array}{c}\text { Cyana- } \\
\text { zine } \\
(\mu g / L)\end{array}$ & $\begin{array}{l}\text { EPTC } \\
(\mu g / L)\end{array}$ & $\begin{array}{c}\text { Triflura- } \\
\text { lin } \\
(\mu g / L)\end{array}$ & $\begin{array}{c}\text { Trial- } \\
\text { late } \\
(\mu g / L)\end{array}$ & $\begin{array}{l}\text { Benflu- } \\
\text { ralin } \\
(\mu g / L)\end{array}$ & $\begin{array}{c}\text { Napro- } \\
\text { pamide } \\
(\mu g / L)\end{array}$ & $\begin{array}{l}\text { Pendi- } \\
\text { methalin } \\
(\mu \mathrm{g} / \mathrm{L})\end{array}$ & $\begin{array}{l}\text { Propa- } \\
\text { chlor } \\
(\mu g / L)\end{array}$ & $\begin{array}{l}\text { Tebuth- } \\
\text { iuron } \\
(\mu \mathrm{g} / \mathrm{L})\end{array}$ & $\begin{array}{l}\text { Metri- } \\
\text { buzin } \\
(\mu g / L)\end{array}$ \\
\hline \multicolumn{11}{|l|}{ Illinois } \\
\hline ILO4 & $<0.01$ & $<0.002$ & $<0.008$ & $<0.003$ & $<0.005$ & $<0.002$ & $<0.01$ & $<0.002$ & 0.05 & $<0.01$ \\
\hline IL09 & $<.01$ & $<.002$ & $<.008$ & $<.003$ & $<.005$ & $<.002$ & $<.01$ & $<.002$ & $<.01$ & $<.01$ \\
\hline IL13 & $<.01$ & $<.002$ & $<.008$ & $<.003$ & $<.005$ & $<.002$ & $<.01$ & $<.002$ & $<.01$ & $<.01$ \\
\hline IL24 & $<.01$ & $<.002$ & $<.008$ & $<.003$ & $<.005$ & $<.002$ & $<.01$ & $<.002$ & $<.01$ & $<.01$ \\
\hline IL25 & $<.01$ & $<.002$ & $<.008$ & $<.003$ & $<.005$ & $<.002$ & $<.01$ & $<.002$ & $<.01$ & $<.01$ \\
\hline IL26 & $<.01$ & $<.002$ & $<.008$ & $<.003$ & $<.005$ & $<.002$ & $<.01$ & $<.002$ & $<.01$ & $<.01$ \\
\hline IL34 & $<.01$ & $<.002$ & $<.008$ & $<.003$ & $<.005$ & $<.002$ & $<.01$ & $<.002$ & $<.01$ & $<.01$ \\
\hline IL41 & $<.01$ & $<.002$ & $<.008$ & $<.003$ & $<.005$ & $<.002$ & $<.01$ & $<.002$ & $<.01$ & $<.01$ \\
\hline
\end{tabular}

Indiana

$\begin{array}{lllllllllll}\text { IN06 } & <.01 & <.002 & <.008 & <.003 & <.005 & <.002 & <.01 & <.002 & <.01 & <.01 \\ \text { IN14 } & <.01 & <.002 & <.008 & <.003 & <.005 & <.002 & <.01 & <.002 & <.01< \\ \text { IN17 } & <.01 & .002 & <.008 & <.003 & <.005 & <.002 & <.01 & .002 & <.01<\end{array}$

lowa

$\begin{array}{llllllllllll}\text { IA05 } & <.01 & <.002 & <.008 & <.003 & <.005 & <.002 & <.01 & <.002 & <.01 & .01 \\ \text { IA15 } & .02 & <.002 & <.008 & <.003 & <.005 & <.002 & <.01 & <.002 & <.01 & <.01 \\ \text { IA18 } & <.01 & .003 & <.008 & <.003 & <.005 & <.002 & <.01 & <.002 & <.01 & <.01 \\ \text { IA19 } & <.01 & <.002 & <.008 & <.003 & <.005 & <.002 & <.01 & <.002 & <.01 & <.01 \\ \text { IA21 } & <.01 & <.002 & <.008 & <.003 & <.005 & <.002 & <.01 & <.002 & <.01 & <.01 \\ \text { IA25 } & <.01 & <.002 & <.008 & <.003 & <.005 & <.002 & <.01 & <.002 & <.01 & <.01 \\ \text { IA26 } & <.01 & <.002 & <.008 & <.003 & <.005 & <.002 & <.01 & <.002 & <.01 & <.01 \\ \text { IA28 } & <.01 & <.002 & <.008 & <.003 & <.005 & <.002 & <.01 & <.002 & <.01 & <.01 \\ \text { IA32 } & <.01 & <.002 & <.008 & <.003 & <.005 & <.002 & <.01 & <.002 & <.01 & <.01 \\ \text { IA34 } & <.01 & <.002 & <.008 & <.003 & <.005 & <.002 & <.01 & <.002 & <.01 & <.01\end{array}$


Table 4. Water-quality data from sensitive analytical methods of water samples for selected pesticides and pesticide metabolites, 1992-Continued

\begin{tabular}{|c|c|c|c|c|c|c|c|c|c|c|}
\hline $\begin{array}{c}\text { Site } \\
\text { identifier } \\
\text { (figs. 1-2) }\end{array}$ & $\begin{array}{c}\text { Atrazine } \\
(\mu g / L)\end{array}$ & $\begin{array}{c}\text { DEA } \\
(\mu g / L)\end{array}$ & $\begin{array}{c}\text { 2,6-Die- } \\
\text { thaniline } \\
(\mu \mathrm{g} / \mathrm{L})\end{array}$ & $\begin{array}{c}\text { Sima- } \\
\text { zine } \\
(\mu g / L)\end{array}$ & $\begin{array}{l}\text { Metol- } \\
\text { achlor } \\
(\mu g / L)\end{array}$ & $\begin{array}{c}\text { Pro- } \\
\text { meton } \\
(\mu \mathrm{g} / \mathrm{L})\end{array}$ & $\begin{array}{c}\text { P,P' } \\
\text { DDE } \\
(\mu g / L)\end{array}$ & $\begin{array}{l}\text { Ala- } \\
\text { chlor } \\
(\mu \mathrm{g} / \mathrm{L})\end{array}$ & $\begin{array}{c}\text { Chlor- } \\
\text { pyrifos } \\
(\mu g / L)\end{array}$ & $\begin{array}{l}\text { Ethal- } \\
\text { fluralin } \\
(\mu \mathrm{g} / \mathrm{L})\end{array}$ \\
\hline MI13 & .130 & .009 & $<.002$ & .005 & $<.002$ & $<.01$ & $<.008$ & $<.003$ & $<.002$ & $<.005$ \\
\hline
\end{tabular}

Minnesota

$\begin{array}{lrrrrrrrrrr}\text { MN02 } & .046 & .006 & <.002 & <.005 & <.002 & <.01 & <.008 & <.003 & <.002 & <.005 \\ \text { MN14 } & .030 & .007 & <.002 & <.005 & <.002 & <.01 & <.008 & .003 & <.002 & <.005 \\ \text { MN15 } & .013 & .007 & <.002 & <.005 & .013 & <.01 & <.008 & <.003 & <.002 & <.005\end{array}$

Missouri

$\begin{array}{rrrrrrrrrrr}\text { M006 } & <.005 & <.003 & <.002 & <.005 & .080 & <.01 & <.008 & .662 & .024 & <.005 \\ \text { M011 } & .086 & .005 & <.002 & <.005 & <.002 & <.01 & <.008 & <.003 & <.002 & <.005\end{array}$

\section{Nebraska}

$\begin{array}{lrrrrrrrrrr}\text { NE05 } & .140 & .036 & <.002 & <.005 & .003 & .47 & .016 & <.003 & .008 & .012 \\ \text { NE15 } & .048 & .009 & <.002 & <.005 & <.002 & <.01 & .030 & <.003 & .010 & .014 \\ \text { NE19 } & .840 & .140 & <.002 & .011 & <.002 & <.01 & <.008 & <.003 & <.002 & <.005 \\ \text { NE25 } & .040 & <.003 & <.002 & <.005 & <.002 & .06 & <.008 & <.003 & <.002 & <.005 \\ \text { NE27 } & .120 & .013 & .002 & .010 & <.002 & <.01 & <.008 & <.003 & <.002 & <.005 \\ \text { NE36 } & .120 & .009 & <.002 & <.005 & <.002 & <.01 & <.008 & <.003 & <.002 & <.005 \\ \text { NE40 } & <.005 & <.003 & <.002 & <.005 & <.002 & <.01 & .013 & <.003 & .005 & .011 \\ \text { NE41 } & .370 & .033 & <.002 & .077 & .018 & .96 & <.008 & <.003 & <.002 & <.005\end{array}$

Ohio

$\begin{array}{lllllllllll}\text { OH07 } & .003 & <.003 & <.002 & .004 & <.002 & <.01 & .001 & <.003 & <.002 & <.005 \\ \text { OH13 } & .003 & <.003 & <.002 & .003 & <.002 & <.01 & <.008 & <.003 & <.002 & <.005 \\ \text { OH16 } & .003 & .002 & <.002 & .002 & <.002 & <.01 & <.008 & <.003 & <.002 & <.005 \\ \text { OH18 } & .003 & <.003 & <.002 & .002 & <.002 & <.01 & <.008 & <.003 & <.002 & <.005 \\ \text { OH19 } & .003 & <.003 & <.002 & .003 & <.002 & <.01 & <.008 & <.003 & <.002 & <.005 \\ \text { OH25 } & .003 & <.003 & <.002 & .002 & <.002 & <.01 & <.008 & <.003 & <.002 & <.005\end{array}$


Table 4. Water-quality data from sensitive analytical methods of water samples for selected pesticides and pesticide metabolites, 1992-Continued

\begin{tabular}{|c|c|c|c|c|c|c|c|c|c|c|}
\hline $\begin{array}{c}\text { Site } \\
\text { identifier } \\
\text { (figs. 1-2) }\end{array}$ & $\begin{array}{c}\text { Cyana- } \\
\text { zine } \\
(\mu g / L)\end{array}$ & $\begin{array}{l}\text { EPTC } \\
(\mu g / L)\end{array}$ & $\begin{array}{l}\text { Triflura- } \\
\text { lin } \\
(\mu g / L)\end{array}$ & $\begin{array}{c}\text { Trial- } \\
\text { late } \\
(\mu g / L)\end{array}$ & $\begin{array}{c}\text { Benflu- } \\
\text { ralin } \\
(\mu g / L)\end{array}$ & $\begin{array}{c}\text { Napro- } \\
\text { pamide } \\
(\mu g / L)\end{array}$ & $\begin{array}{c}\text { Pendi- } \\
\text { methalin } \\
(\mu g / L)\end{array}$ & $\begin{array}{l}\text { Propa- } \\
\text { chlor } \\
(\mu g / L)\end{array}$ & $\begin{array}{l}\text { Tebuth- } \\
\text { iuron } \\
(\mu g / L)\end{array}$ & $\begin{array}{l}\text { Metri- } \\
\text { buzin } \\
(\mu g / L)\end{array}$ \\
\hline \multicolumn{11}{|l|}{ Michigan } \\
\hline MI13 & .01 & $<.002$ & $<.008$ & $<.003$ & $<.005$ & $<.002$ & $<.01$ & $<.002$ & $<.01$ & $<.01$ \\
\hline
\end{tabular}

Minnesota

$\begin{array}{lllllllllll}\text { MN02 } & <.01 & <.002 & <.008 & <.003 & <.005 & <.002 & <.01 & <.002 & <.01 & <.01 \\ \text { MN14 } & <.01 & <.002 & <.008 & <.003 & <.005 & <.002 & <.01 & <.002 & <.01 \quad<\quad<.01 \\ \text { MN15 } & <.01 & <.002 & <.008 & <.003 & <.005 & <.002 & <.01 & <.002 & <.01 \quad & <.01\end{array}$

Missouri

$\begin{array}{lllllllllll}\text { M006 } & <.01 & <.002 & <.008 & <.003 & <.005 & <.002 & <.01 & <.002 & <.01 & <.01 \\ \text { M011 } & <.01 & <.002 & <.008 & <.003 & <.005 & <.002 & <.01 & <.002 & <.01 & <.01\end{array}$

Nebraska

$\begin{array}{lllllllllll}\text { NE05 } & <.01 & <.002 & .008 & .004 & <.005 & <.002 & <.01 & <.002 & <.01 & <.01 \\ \text { NE15 } & <.01 & <.002 & .016 & .007 & .018 & <.002 & .01 & <.002 & <.01 & <.01 \\ \text { NE19 } & <.01 & <.002 & <.008 & <.003 & <.005 & <.002 & <.01 & <.002 & <.01 & <.01 \\ \text { NE25 } & <.01 & <.002 & <.008 & <.003 & <.005 & <.002 & <.01 & <.002 & <.01 & <.01 \\ \text { NE27 } & <.01 & <.002 & <.008 & <.003 & <.005 & <.002 & <.01 & <.002 & <.01 & <.01 \\ \text { NE36 } & <.01 & <.002 & <.008 & <.003 & <.005 & <.002 & <.01 & <.002 & <.01 & <.01 \\ \text { NE40 } & .01 & <.002 & <.008 & <.003 & <.005 & <.002 & <.01 & <.002 & <.01 & <.01 \\ \text { NE41 } & <.01 & <.002 & <.008 & <.003 & <.005 & <.002 & <.01 & <.002 & <.01 & <.01\end{array}$

Ohio

$\begin{array}{lllllllllll}\text { OH07 } & <.01 & <.002 & <.008 & <.003 & <.005 & <.002 & <.01 & <.002 & <.01 & <.01 \\ \text { OH13 } & <.01 & <.002 & <.008 & <.003 & <.005 & <.002 & <.01 & <.002 & <.01 & <.01 \\ \text { OH16 } & <.01 & <.002 & <.008 & <.003 & <.005 & <.002 & <.01 & <.002 & <.01 & <.01 \\ \text { OH18 } & <.01 & <.002 & <.008 & <.003 & <.005 & <.002 & <.01 & <.002 & <.01 & <.01 \\ \text { OH19 } & <.01 & <.002 & <.008 & <.003 & <.005 & <.002 & <.01 & <.002 & <.01 & <.01 \\ \text { OH25 } & <.01 & <.002 & <.008 & <.003 & <.005 & <.002 & <.01 & <.002 & <.01 & <.01\end{array}$


Table 4. Water-quality data from sensitive analytical methods of water samples for selected pesticides and pesticide metabolites, 1992-Continued

\begin{tabular}{|c|c|c|c|c|c|c|c|c|c|c|}
\hline $\begin{array}{c}\text { Site } \\
\text { identifier } \\
\text { (figs. 1-2) }\end{array}$ & $\begin{array}{c}\text { Atrazine } \\
(\mu g / L)\end{array}$ & $\begin{array}{c}\text { DEA } \\
(\mu g / L)\end{array}$ & $\begin{array}{c}\text { 2,6-Die- } \\
\text { thani- } \\
\text { line }(\mu g / L)\end{array}$ & $\begin{array}{c}\text { Sima- } \\
\text { zine } \\
(\mu g / L)\end{array}$ & $\begin{array}{l}\text { Metol- } \\
\text { achlor } \\
(\mu \mathrm{g} / L)\end{array}$ & $\begin{array}{l}\text { Pro- } \\
\text { meton } \\
(\mu g / L)\end{array}$ & $\begin{array}{c}\text { P,P' } \\
\text { DDE } \\
(\mu g / L)\end{array}$ & $\begin{array}{l}\text { Ala- } \\
\text { chlor } \\
(\mu g / L)\end{array}$ & $\begin{array}{c}\text { Chlor- } \\
\text { pyrifos } \\
(\mu g / L)\end{array}$ & $\begin{array}{c}\text { Ethal- } \\
\text { fluralin } \\
(\mu g / L)\end{array}$ \\
\hline $\mathrm{OH} 26$ & .004 & $<.003$ & $<.002$ & .004 & $<.002$ & $<.01$ & $<.008$ & $<.003$ & $<.002$ & $<.005$ \\
\hline \multicolumn{11}{|l|}{ Wisconsin } \\
\hline WI02 & $<.005$ & .004 & $<.002$ & $<.005$ & $<.002$ & $<.01$ & $<.008$ & $<.003$ & $<.002$ & $<.005$ \\
\hline WI11 & .079 & .035 & .005 & $<.005$ & $<.002$ & $<.01$ & $<.008$ & $<.003$ & $<.002$ & $<.005$ \\
\hline
\end{tabular}


Table 4. Water-quality data from sensitive analytical methods of water samples for selected pesticides and pesticide metabolites, 1992-Continued

\begin{tabular}{|c|c|c|c|c|c|c|c|c|c|c|}
\hline $\begin{array}{c}\text { Site } \\
\text { identifier } \\
\text { (figs. 1-2) }\end{array}$ & $\begin{array}{l}\text { Cyana- } \\
\text { zine } \\
(\mu g / L)\end{array}$ & $\begin{array}{l}\text { EPTC } \\
(\mu g / L)\end{array}$ & $\begin{array}{l}\text { Triflura- } \\
\text { lin } \\
(\mu g / L)\end{array}$ & $\begin{array}{l}\text { Trial- } \\
\text { late } \\
(\mu g / L)\end{array}$ & $\begin{array}{c}\text { Benflu- } \\
\text { ralin } \\
(\mu g / L)\end{array}$ & $\begin{array}{c}\text { Napro- } \\
\text { pamide } \\
(\mu g / L)\end{array}$ & $\begin{array}{c}\text { Pendi- } \\
\text { methalin } \\
(\mu g / L)\end{array}$ & $\begin{array}{l}\text { Propa- } \\
\text { chlor } \\
(\mu g / L)\end{array}$ & $\begin{array}{l}\text { Tebuth- } \\
\text { iuron } \\
(\mu g / L)\end{array}$ & $\begin{array}{l}\text { Metri- } \\
\text { buzin } \\
(\mu g / L)\end{array}$ \\
\hline \multicolumn{11}{|c|}{ Ohio (Continued) } \\
\hline $\mathrm{OH} 26$ & $<.01$ & $<.002$ & $<.008$ & $<.003$ & $<.005$ & .008 & $<.01$ & $<.002$ & $<.01$ & $<.01$ \\
\hline $\mathrm{OH} 27$ & $<.01$ & $<.002$ & $<.008$ & $<.003$ & $<.005$ & $<.002$ & $<.01$ & $<.002$ & $<.01$ & $<.01$ \\
\hline \multicolumn{11}{|l|}{ Wisconsin } \\
\hline WI02 & $<.01$ & $<.002$ & $<.008$ & $<.003$ & $<.005$ & $<.002$ & $<.01$ & $<.002$ & $<.01$ & $<.01$ \\
\hline WI03 & $<.01$ & $<.002$ & $<.008$ & $<.003$ & $<.005$ & $<.002$ & $<.01$ & $<.002$ & $<.01$ & $<.01$ \\
\hline WI05 & $<.01$ & $<.002$ & $<.008$ & $<.003$ & $<.005$ & $<.002$ & $<.01$ & $<.002$ & $<.01$ & $<.01$ \\
\hline WI07 & $<.01$ & $<.002$ & $<.008$ & $<.003$ & $<.005$ & $<.002$ & $<.01$ & $<.002$ & $<.01$ & $<.01$ \\
\hline WI11 & $<.01$ & $<.002$ & $<.008$ & $<.003$ & $<.005$ & $<.002$ & $<.01$ & $<.002$ & $<.01$ & $<.01$ \\
\hline
\end{tabular}

' Reporting limits of compounds not detected: alpha BHC (0.002), butylate (0.002), carbaryl (0.01), carbofuran (0.01), dacthal (0.002), diazinon (0.002), dieldrin (0.002), dimethoate (0.02), disulfoton (0.017), ethoprop $(0.003)$, fonofos $(0.003)$, linuron $(0.01)$, lindane $(0.008)$, malathion $(0.005)$, methyl parathion (0.008), methyl azinphos (0.005), molinate $(0.004)$, parathion $(0.005)$, pebulate $(0.005)$, permethrin $(0.01)$, phorate $(0.003)$, $(0.002)$, pronamide $(0.004)$, propargite $(0.02)$, propanil $(u .005)$, terbacil $(0.01)$, terbufos $(0.013)$, thiobencarb $(0.002)$ 
Table 5. Water-quality data from laboratory analysis of water samples for selected chlorophenoxy-acid and other miscellaneous herbicides, 1992

$[\mu \mathrm{g} / \mathrm{L}$, micrograms per liter; DCPA, DCPA-acid metabolite; <, less than]

\begin{tabular}{|c|c|c|c|c|c|c|c|c|c|}
\hline $\begin{array}{c}\text { Site } \\
\text { indentifier } \\
\text { (figs. 1-2) }\end{array}$ & $\begin{array}{c}\text { Acifluor- } \\
\text { fen, } \\
(\mu g / L)\end{array}$ & $\begin{array}{c}\text { Bentazon } \\
(\mu g / L)\end{array}$ & $\begin{array}{l}2,4-D \\
(\mu g / L)\end{array}$ & $\begin{array}{l}\text { DCPA } \\
(\mu \mathrm{g} / \mathrm{L})\end{array}$ & $\begin{array}{c}\text { Dicamba } \\
(\mu g / L)\end{array}$ & $\begin{array}{l}2,4-D P \\
(\mu g / L)\end{array}$ & $\begin{array}{l}\text { Picloram } \\
(\mu g / L)\end{array}$ & $\begin{array}{l}\text { Silvex } \\
(\mu g / L)\end{array}$ & $\begin{array}{r}2,4,5-T \\
(\mu g / L)\end{array}$ \\
\hline \multicolumn{10}{|l|}{ Illinois } \\
\hline IL04 & $<0.01$ & $<0.10$ & $<0.01$ & $<0.01$ & $<0.01$ & $<0.01$ & $<0.01$ & $<0.01$ & $<0.01$ \\
\hline IL05 & $<.01$ & $<.10$ & $<.01$ & $<.01$ & $<.01$ & $<.01$ & $<.01$ & $<.01$ & $<.01$ \\
\hline IL09 & $<.01$ & $<.10$ & $<.01$ & $<.01$ & $<.01$ & $<.01$ & $<.01$ & $<.01$ & $<.01$ \\
\hline IL13 & $<.01$ & $<.10$ & $<.01$ & $<.01$ & $<.01$ & $<.01$ & $<.01$ & $<.01$ & $<.01$ \\
\hline IL25 & $<.01$ & $<.10$ & $<.01$ & $<.01$ & $<.01$ & $<.01$ & $<.01$ & $<.01$ & $<.01$ \\
\hline IL26 & $<.01$ & $<.10$ & $<.01$ & $<.01$ & $<.01$ & $<.01$ & $<.01$ & $<.01$ & $<.01$ \\
\hline IL34 & $<.01$ & $<.10$ & $<.01$ & $<.01$ & $<.01$ & $<.01$ & $<.01$ & $<.01$ & $<.01$ \\
\hline IL41 & $<.01$ & $<.10$ & $<.01$ & $<.01$ & $<.01$ & $<.01$ & $<.01$ & $<.01$ & $<.01$ \\
\hline IL42 & $<.01$ & $<.10$ & $<.01$ & $<.01$ & $<.01$ & $<.01$ & $<.01$ & $<.01$ & $<.01$ \\
\hline IL48 & $<.01$ & $<.10$ & $<.01$ & $<.01$ & $<.01$ & $<.01$ & $<.01$ & $<.01$ & $<.01$ \\
\hline
\end{tabular}

lowa

$\begin{array}{llrrrrrrrr}\text { IA05 } & <.01 & <.10 & <.01 & .49 & <.01 & <.01 & <.01 & <.01 & <.01 \\ \text { IA13 } & <.01 & <.10 & .10 & <.01 & <.01 & <.01 & <.01 & <.01 & .02 \\ \text { IA15 } & <.01 & <.10 & .17 & <.01 & .01 & <.01 & <.01 & <.01 & <.01 \\ \text { IA19 } & <.01 & <.10 & <.01 & .03 & <.01 & <.01 & <.01 & <.01 & <.01 \\ \text { IA21 } & <.01 & <.10 & <.01 & <.01 & <.01 & <.01 & <.01 & <.01 & <.01 \\ \text { IA24 } & <.01 & <.10 & <.01 & <.01 & <.01 & <.01 & <.01 & <.01 & <.01 \\ \text { IA25 } & <.01 & <.10 & <.01 & <.01 & .01 & <.01 & <.01 & <.01 & <.01 \\ \text { IA26 } & <.01 & <.10 & <.01 & <.01 & <.01 & <.01 & <.01 & <.01 & <.01 \\ \text { IA34 } & <.01 & <.10 & <.01 & <.01 & <.01 & <.01 & <.01 & <.01 & <.01\end{array}$

$\begin{array}{llllllllll}\text { Indiana } & & & & & & & & \\ \text { IN04 } & <.01 & <.10 & <.01 & <.01 & <.01 & <.01 & <.01 & <.01 & <.01 \\ \text { IN06 } & <.01 & <.10 & <.01 & <.01 & <.01 & <.01 & <.01 & <.01 & <.01 \\ \text { IN14 } & <.01 & <.10 & <.01 & <.01 & <.01 & <.01 & <.01 & <.01 & <.01 \\ \text { IN28 } & <.01 & <.10 & <.01 & <.01 & <.01 & <.01 & <.01 & <.01 & <.01\end{array}$


Table 5. Water-quality data from laboratory analyses of water samples for selected chlorophenoxy-acid and other miscellaneous herbicides, 1992-Continued

\begin{tabular}{|c|c|c|c|c|c|c|c|c|c|}
\hline $\begin{array}{c}\text { Site } \\
\text { indentifier } \\
\text { (figs. 1-2) }\end{array}$ & $\begin{array}{c}\text { Acifluor- } \\
\text { fen, } \\
(\mu g / L)\end{array}$ & $\begin{array}{c}\text { Bentazon } \\
(\mu g / L)\end{array}$ & $\begin{array}{l}2,4-D \\
(\mu g / L)\end{array}$ & $\begin{array}{l}\text { DCPA } \\
(\mu g / L)\end{array}$ & $\begin{array}{c}\text { Dicamba } \\
(\mu g / L)\end{array}$ & $\begin{array}{l}\text { 2,4-DP } \\
(\mu g / L)\end{array}$ & $\begin{array}{l}\text { Picloram } \\
(\mu \mathrm{g} / \mathrm{L})\end{array}$ & $\begin{array}{l}\text { Silvex } \\
(\mu g / L)\end{array}$ & $\begin{array}{r}2,4,5-T \\
(\mu g / L)\end{array}$ \\
\hline \multicolumn{10}{|l|}{ Michigan } \\
\hline MI13 & $<.01$ & $<.10$ & $<.01$ & .01 & $<.01$ & $<.01$ & $<.01$ & $<.01$ & $<.01$ \\
\hline
\end{tabular}

Minnesota

$\begin{array}{llllllllll}\text { MN02 } & <.01 & <.10 & <.01 & <.01 & <.01 & <.01 & <.01 & <.01< & <.01 \\ \text { MN14 } & <.01 & <.10 & <.01 & <.01 & <.01 & <.01 & <.01< & <.01<\end{array}$

Missouri

$\begin{array}{llllllllll}\text { M006 } & <.01 & <.10 & .89 & <.01 & <.01 & <.01 & <.01 & <.01 & <.01 \\ \text { M011 } & <.01 & <.10 & <.01 & <.01 & <.01 & <.01 & <.01 & <.01<\end{array}$

Nebraska

$\begin{array}{llllllllll}\text { NE05 } & <.01 & <.10 & <.01 & .47 & <.01 & <.01 & <.01 & <.01 & <.01 \\ \text { NE15 } & <.01 & <.10 & <.01 & <.01 & <.01 & <.01 & <.01 & <.01 & <.01 \\ \text { NE17 } & <.01 & <.10 & <.01 & <.01 & <.01 & <.01 & <.01 & <.01 & <.01 \\ \text { NE19 } & <.01 & <.10 & <.01 & <.01 & <.01 & <.01 & <.01 & <.01 & <.01 \\ \text { NE24 } & <.01 & <.10 & <.01 & <.01 & <.01 & <.01 & <.01 & <.01 & <.01 \\ \text { NE25 } & <.01 & <.10 & <.01 & <.01 & <.01 & <.01 & <.01 & <.01 & <.01 \\ \text { NE27 } & <.01 & <.10 & <.01 & .01 & <.01 & <.01 & .01 & <.01 & <.01 \\ \text { NE36 } & <.01 & <.10 & <.01 & <.01 & <.01 & <.01 & <.01 & <.01 & <.01 \\ \text { NE39 } & <.01 & <.10 & <.01 & .01 & <.01 & <.01 & <.01 & <.01 & <.01 \\ \text { NE41 } & <.01 & <.10 & <.01 & <.01 & <.01 & <.01 & .03 & <.01 & <.01 \\ \text { NE42 } & <.01 & <.10 & <.01 & 2.22 & <.01 & <.01 & <.01 & <.01 & <.01\end{array}$

Ohio

$\mathrm{OH} 07$

$<.01<.10<.01<01$

$<.01$

$<.01$

$<.01$ 
Table 5. Water-quality data from laboratory analyses of water samples for selected chlorophenoxy-acid and other miscellaneous herbicides, 1992-Continued

\begin{tabular}{lccccccccc}
\hline $\begin{array}{c}\text { Site } \\
\text { indentifier } \\
\text { (figs. 1-2) }\end{array}$ & $\begin{array}{c}\text { Acifluor- } \\
\text { fen, } \\
(\mu \mathrm{g} / \mathrm{L})\end{array}$ & $\begin{array}{c}\text { Bentazon } \\
(\mu \mathrm{g} / \mathrm{L})\end{array}$ & $\begin{array}{c}\mathbf{2 , 4 - D} \\
(\mu \mathrm{g} / \mathrm{L})\end{array}$ & $\begin{array}{c}\text { DCPA } \\
(\mu \mathrm{g} / \mathrm{L})\end{array}$ & $\begin{array}{c}\text { Dicamba } \\
(\mu \mathrm{g} / \mathrm{L})\end{array}$ & $\begin{array}{c}\mathbf{2 , 4 - D P} \\
(\mu \mathrm{g} / \mathrm{L})\end{array}$ & $\begin{array}{c}\text { Picloram } \\
(\mu \mathrm{g} / \mathrm{L})\end{array}$ & $\begin{array}{c}\text { Silvex } \\
(\mu \mathrm{g} / \mathrm{L})\end{array}$ & $\begin{array}{c}\mathbf{2 , 4 , 5 - T} \\
(\mu \mathrm{g} / \mathrm{L})\end{array}$ \\
\hline Wisconsin & & & & & & & & & \\
W102 & $<.01$ & $<.10$ & $<.01$ & $<.01$ & $<.01$ & $<.01$ & $<.01$ & $<.01$ & $<.01$ \\
WI03 & $<.01$ & $<.10$ & $<.01$ & $<.01$ & $<.01$ & $<.01$ & $<.01$ & $<.01$ & $<.01$ \\
W105 & $<.01$ & $<.10$ & $<.01$ & $<.01$ & $<.01$ & $<.01$ & $<.01$ & $<.01$ & $<.01$ \\
WI10 & $<.01$ & $<.10$ & $<.01$ & $<.01$ & $<.01$ & $<.01$ & $<.01$ & $<.01$ & $<.01$ \\
WI11 & $<.01$ & $<.10$ & $<.01$ & $<.01$ & $<.01$ & $<.01$ & $<.01$ & $<.01$ & $<.01$ \\
\hline
\end{tabular}


Table 6. Water-quality data from laboratory analyses of water samples for detected volatile organic compounds and methylene-blue active substances, 1992

[ $\mu \mathrm{g} / \mathrm{L}$, micrograms per liter; MBAS, methylene-blue active substances; <, less than; - , no data]

\begin{tabular}{|c|c|c|c|c|}
\hline $\begin{array}{c}\text { Site } \\
\text { identifier } \\
\text { (figs. 1-2) }\end{array}$ & $\begin{array}{l}\text { 1,2-di- } \\
\text { chloro- } \\
\text { ethane } \\
\text { ( } \mathrm{mg} / \mathrm{L} \text { ) }\end{array}$ & $\begin{array}{c}\text { Chloro- } \\
\text { form } \\
(\mu \mathrm{g} / \mathrm{L})\end{array}$ & $\begin{array}{l}\text { MBAS } \\
(\mu g / L)\end{array}$ & $\begin{array}{c}\text { Tetra- } \\
\text { chloro- } \\
\text { ethylene } \\
(\mu \mathrm{g} / \mathrm{L})\end{array}$ \\
\hline \multicolumn{5}{|l|}{ Illinois } \\
\hline IL04 & $<0.2$ & $<0.2$ & 0.04 & $<0.2$ \\
\hline IL05 & $<.2$ & $<.2$ & .04 & $<.2$ \\
\hline ILO9 & $<.2$ & $<.2$ & .22 & $<.2$ \\
\hline IL13 & $<.2$ & .7 & .06 & .9 \\
\hline IL25 & $<.2$ & $<.2$ & .04 & $<.2$ \\
\hline IL26 & $<.2$ & $<.2$ & .04 & $<.2$ \\
\hline IL34 & $<.2$ & $<.2$ & .03 & $<.2$ \\
\hline IL41 & $<.2$ & $<.2$ & .03 & $<.2$ \\
\hline IL42 & $<.2$ & $<.2$ & .02 & $<.2$ \\
\hline IL48 & $<.2$ & $<.2$ & .01 & $<.2$ \\
\hline \multicolumn{5}{|l|}{ Indiana } \\
\hline IN04 & $<.2$ & $<.2$ & .03 & $<.2$ \\
\hline IN06 & $<.2$ & $<.2$ & .07 & $<.2$ \\
\hline IN14 & $<.2$ & $<.2$ & .08 & $<.2$ \\
\hline IN28 & $<.2$ & $<.2$ & .06 & $<.2$ \\
\hline \multicolumn{5}{|l|}{ lowa } \\
\hline IA05 & $<.2$ & $<.2$ & .06 & $<.2$ \\
\hline IA13 & $<.2$ & $<.2$ & .02 & $<.2$ \\
\hline IA 15 & $<.2$ & $<.2$ & .17 & $<.2$ \\
\hline IA 19 & $<.2$ & $<.2$ & .06 & $<.2$ \\
\hline IA21 & $<.2$ & $<.2$ & .06 & $<.2$ \\
\hline IA24 & $<.2$ & $<.2$ & .02 & $<.2$ \\
\hline IA25 & $<.2$ & $<.2$ & .04 & $<.2$ \\
\hline IA26 & $<.2$ & $<.2$ & .07 & $<.2$ \\
\hline IA34 & $<.2$ & $<.2$ & .02 & $<.2$ \\
\hline
\end{tabular}

\begin{tabular}{ccccc}
\hline $\begin{array}{c}\text { Site } \\
\text { identifier } \\
\text { (figs. 1-2) }\end{array}$ & $\begin{array}{c}\text { 1,2-di- } \\
\text { chloro- } \\
\text { ethane } \\
(\mathrm{mg} / \mathrm{L})\end{array}$ & $\begin{array}{c}\text { Chloro- } \\
\text { form } \\
(\mu \mathrm{g} / \mathrm{L})\end{array}$ & $\begin{array}{c}\text { MBAS } \\
(\mu \mathrm{g} / \mathrm{L})\end{array}$ & $\begin{array}{c}\text { Tetra- } \\
\text { chloro- } \\
\text { ethylene } \\
(\mu \mathrm{g} / \mathrm{L})\end{array}$ \\
\hline Michigan & & & & \\
MI13 & $<.2$ & $<.2$ & .03 & $<.2$
\end{tabular}

Minnesota

$\begin{array}{lllll}\text { MN02 } & <.2 & <.2 & .03 & <.2 \\ \text { MN14 } & <.2 & <.2 & .08 & <.2\end{array}$

Missouri

$\begin{array}{lrrrr}\text { MO06 } & <.2 & .2 & .03 & <.2 \\ \text { MO11 } & <.2 & <.2 & .03 & <.2\end{array}$

Nebraska

$\begin{array}{lllll}\text { NE05 } & .2 & <.2 & .12 & 1.1\end{array}$

$\begin{array}{lllll}\mathrm{NE} 15 & <.2 & .2 & .11 & <.2\end{array}$

$\begin{array}{lllll}\mathrm{NE} 17 & <.2 & <.2 & .04 & <.2\end{array}$

$\begin{array}{lllll}N E 19 & <.2 & <.2 & .07 & <.2\end{array}$

$\mathrm{NE} 24<.2<.2 \quad .02 \quad<.2$

$\begin{array}{lllll}\mathrm{NE} 25 & <.2 & <.2 & .02 & <.2\end{array}$

$\begin{array}{llll}\mathrm{NE} 27 & <.2 & <.2 & .02<\end{array}$

$\begin{array}{lllll}\mathrm{NE} 36 & <.2 & .4 & .08 & <.2\end{array}$

$\begin{array}{lllll}N & <39 & <.2 & .01 & <.2\end{array}$

$\begin{array}{lllll}\text { NE41 } & <.2 & .8 & .03 & <.2\end{array}$

$\mathrm{NE} 42<.2 \quad<.2 \quad .05 \quad<.2$

Ohio

$\begin{array}{lllll}\mathrm{OH} 07 & <.2 & <.2 & .02 & <.2\end{array}$ 
Table 6. Water-quality data from laboratory analyses of water samples for detected volatile organic compounds and methylene-blue active substances, 1992-Continued

\begin{tabular}{lcccc}
\hline $\begin{array}{c}\text { Site } \\
\text { identifier } \\
\text { (figs. 1-2) }\end{array}$ & $\begin{array}{c}\text { 1,2-di- } \\
\text { chloro- } \\
\text { ethane } \\
(\mathrm{mg} / \mathrm{L})\end{array}$ & $\begin{array}{c}\text { Chloro- } \\
\text { form } \\
(\mu \mathrm{g} / \mathrm{L})\end{array}$ & $\begin{array}{c}\text { MBAS } \\
(\mu \mathrm{g} / \mathrm{L})\end{array}$ & $\begin{array}{c}\text { Tetra- } \\
\text { chloro- } \\
\text { ethylene } \\
(\mu \mathrm{g} / \mathrm{L})\end{array}$ \\
\hline Wisconsin & & & & \\
WI02 & $<.2$ & $<.2$ & .09 & $<.2$ \\
WI03 & $<.2$ & $<.2$ & .13 & $<.2$ \\
WI05 & $<.2$ & $<.2$ & - & $<.2$ \\
WI10 & $<.2$ & $<.2$ & $<.01$ & $<.2$ \\
WI11 & $<.2$ & $<.2$ & .08 & $<.2$ \\
\hline
\end{tabular}


Table 7. Water-quality data from field measurements and laboratory analyses for inorganic nutrients, 1993 $\left[{ }^{\circ} \mathrm{C}\right.$, degrees Celsius; $\mu \mathrm{S} / \mathrm{cm}$, microsiemens per centimeter at 25 degrees Celsius; $\mathrm{mg} / \mathrm{L}$, milligrams per liter; - , no data; <, less than]

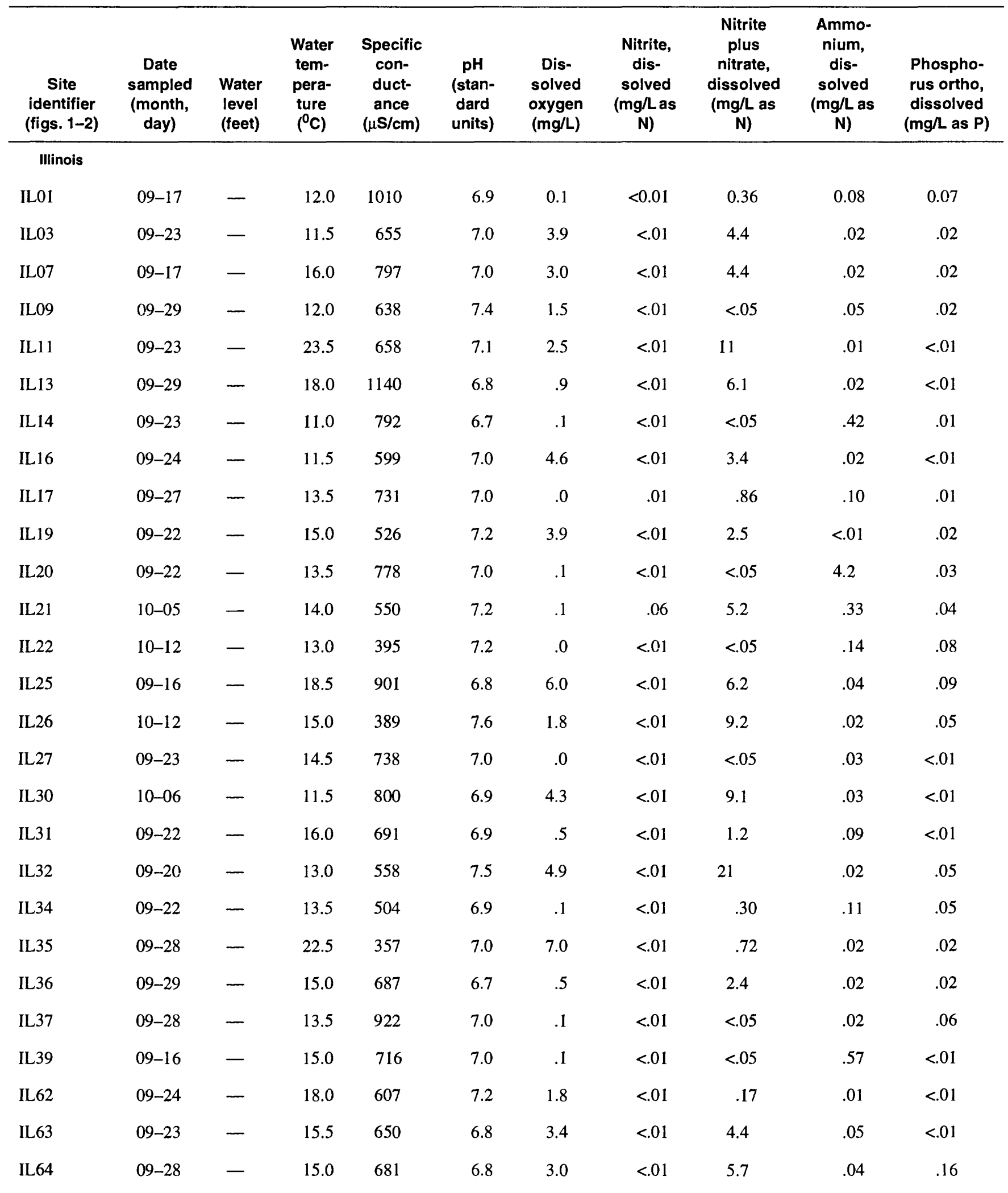


Table 7. Water-quality data from field measurements and laboratory analyses for inorganic nutrients, 1993-Continued

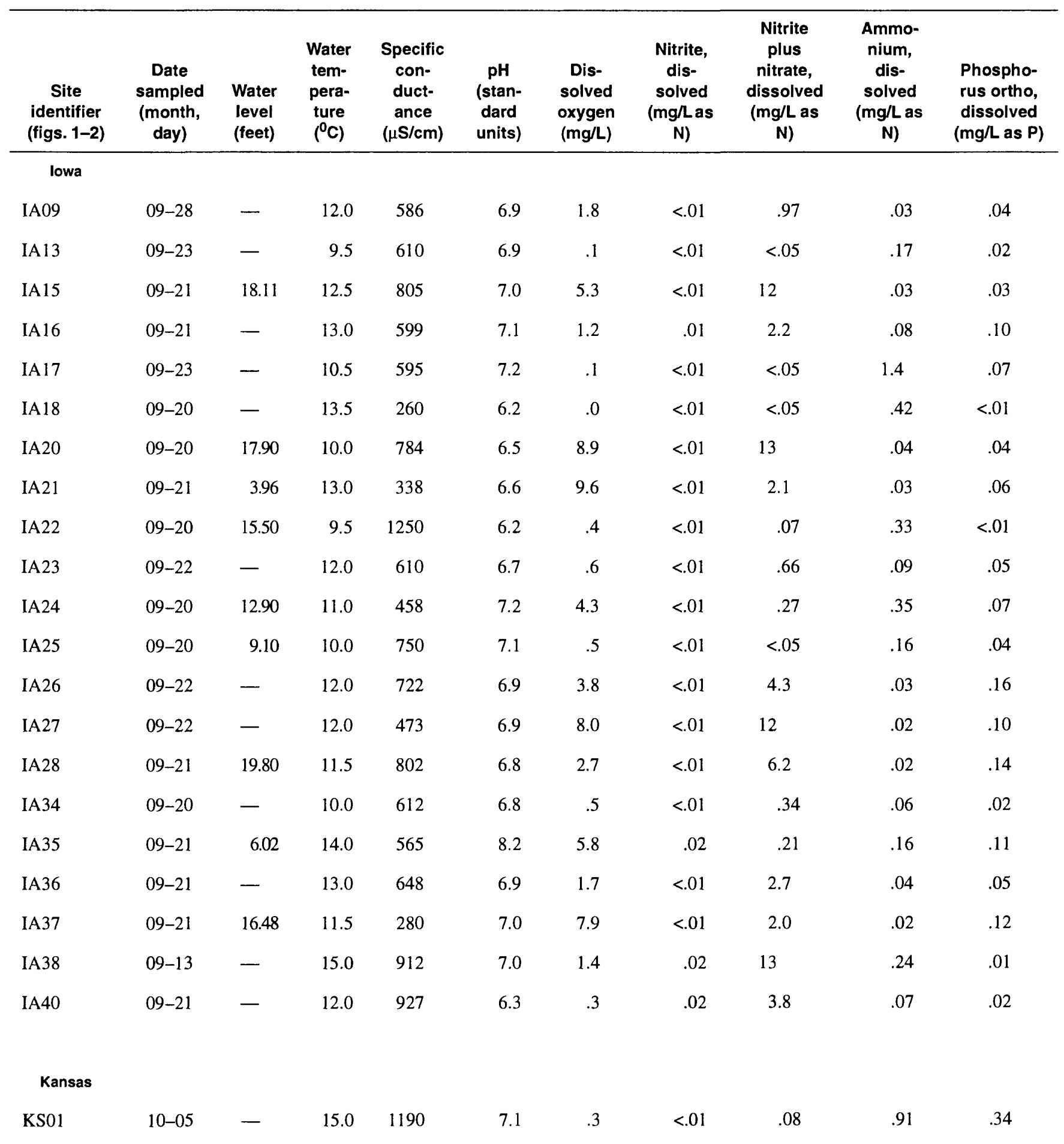


Table 7. Water-quality data from field measurements and laboratory analyses for inorganic nutrients, 1993—Continued

\begin{tabular}{|c|c|c|c|c|c|c|c|c|c|c|}
\hline $\begin{array}{c}\text { Site } \\
\text { identifier } \\
\text { (figs. 1-2) }\end{array}$ & $\begin{array}{c}\text { Date } \\
\text { sampled } \\
\text { (month, } \\
\text { day) }\end{array}$ & $\begin{array}{l}\text { Water } \\
\text { level } \\
\text { (feet) }\end{array}$ & $\begin{array}{l}\text { Water } \\
\text { tem- } \\
\text { pera- } \\
\text { ture } \\
\left({ }^{\circ} \mathrm{C}\right)\end{array}$ & $\begin{array}{c}\text { Specific } \\
\text { con- } \\
\text { duct- } \\
\text { ance } \\
(\mu \mathrm{S} / \mathrm{cm})\end{array}$ & $\begin{array}{c}\text { pH } \\
\text { (stan- } \\
\text { dard } \\
\text { units) }\end{array}$ & $\begin{array}{l}\text { Dis- } \\
\text { solved } \\
\text { oxygen } \\
\text { (mg/L) }\end{array}$ & $\begin{array}{c}\text { Nitrite, } \\
\text { dis- } \\
\text { solved } \\
\text { (mg/Las } \\
\mathrm{N})\end{array}$ & $\begin{array}{l}\text { Nitrite } \\
\text { plus } \\
\text { nitrate, } \\
\text { dissolved } \\
\text { (mg/L as } \\
\mathrm{N} \text { ) }\end{array}$ & $\begin{array}{l}\text { Ammo- } \\
\text { nium, } \\
\text { dis- } \\
\text { solved } \\
\text { (mg/Las } \\
\mathrm{N} \text { ) }\end{array}$ & $\begin{array}{l}\text { Phospho- } \\
\text { rus ortho, } \\
\text { dissolved } \\
\text { (mg/L as P) }\end{array}$ \\
\hline \multicolumn{11}{|l|}{ Minnesota } \\
\hline MN01 & $10-07$ & 52.00 & 12.0 & 713 & 7.2 & .1 & $<.01$ & $<.05$ & 1.1 & .01 \\
\hline MN03 & $10-04$ & 15.18 & 13.0 & 927 & 7.4 & .1 & $<.01$ & $<.05$ & 2.1 & .02 \\
\hline MN04 & $10-06$ & - & 11.0 & 2840 & 6.9 & .1 & $<.01$ & $<.05$ & 2.3 & $<.01$ \\
\hline MN05 & $10-06$ & 8.00 & 10.0 & 486 & 7.6 & 1.0 & .02 & .75 & .02 & $<.01$ \\
\hline MN09 & 09-29 & - & 11.0 & 798 & 7.4 & 3.2 & $<.01$ & .81 & .02 & $<.01$ \\
\hline MN10 & $09-27$ & 21.47 & 8.0 & 720 & 7.2 & 6.4 & $<.01$ & 17 & .03 & .01 \\
\hline MN11 & $09-28$ & 27.52 & 11.0 & 1070 & 7.3 & .2 & $<.01$ & $<.05$ & 1.5 & $<.01$ \\
\hline MN13 & $09-29$ & 12.80 & 9.0 & 1480 & 7.1 & .4 & $<.01$ & $<.05$ & .68 & $<.01$ \\
\hline MN15 & $10-06$ & - & 10.0 & 1160 & 7.2 & .1 & $<.01$ & $<.05$ & .29 & $<.01$ \\
\hline MN16 & $09-29$ & 12.30 & 9.5 & 2020 & 6.9 & .1 & $<.01$ & $<.05$ & .06 & .02 \\
\hline MN18 & $10-05$ & - & 14.5 & 579 & 7.1 & .1 & $<.01$ & $<.05$ & .15 & .06 \\
\hline MN26 & $10-05$ & 15.27 & 12.0 & 1070 & 7.0 & .2 & $<.01$ & 13 & .09 & .02 \\
\hline MN27 & $10-06$ & 7.24 & 13.0 & 1560 & 6.7 & .3 & $<.01$ & $<.05$ & .09 & $<.01$ \\
\hline MN28 & $09-28$ & 9.98 & 12.5 & 1930 & 6.8 & .1 & $<.01$ & $<.05$ & .27 & $<.01$ \\
\hline
\end{tabular}

Missouri

$\begin{array}{lrlllllllrr}\text { MO03 } & 10-04 & - & 14.0 & 738 & 7.2 & .0 & <.01 & <.05 & .26 & <.01 \\ \text { MO04 } & 10-21 & - & 14.5 & 794 & 7.6 & - & <.01 & .62 & .04 & .01 \\ \text { MO05 } & 10-04 & 15.00 & 14.0 & 609 & 7.1 & .6 & <.01 & .48 & .14 & .01 \\ \text { MO06 } & 10-05 & - & 13.5 & 841 & 7.2 & .1 & <.01 & <.05 & .30 & <.01 \\ \text { MO07 } & 10-04 & - & 15.0 & 878 & 7.1 & 5.0 & <.01 & .42 & .15 & .01 \\ \text { MO08 } & 10-05 & - & 13.0 & 931 & 7.2 & .1 & <.01 & <.05 & .75 & .48 \\ \text { MO09 } & 10-04 & - & 14.5 & 715 & 7.1 & .2 & <.01 & .15 & .06 & .03 \\ \text { MO15 } & 9-14 & - & 13.5 & 797 & 7.2 & - & <.01 & .28 & .14 & <.01\end{array}$


Table 7. Water-quality data from field measurements and laboratory analyses for inorganic nutrients, 1993—Continued

\begin{tabular}{|c|c|c|c|c|c|c|c|c|c|c|}
\hline $\begin{array}{c}\text { Site } \\
\text { identifier } \\
\text { (figs. 1-2) }\end{array}$ & $\begin{array}{c}\text { Date } \\
\text { sampled } \\
\text { (month, } \\
\text { day) }\end{array}$ & $\begin{array}{l}\text { Water } \\
\text { level } \\
\text { (feet) }\end{array}$ & $\begin{array}{l}\text { Water } \\
\text { tem- } \\
\text { pera- } \\
\text { ture } \\
\left({ }^{\circ} \mathrm{C}\right)\end{array}$ & $\begin{array}{l}\text { Specific } \\
\text { con- } \\
\text { duct- } \\
\text { ance } \\
(\mu \mathrm{S} / \mathrm{cm})\end{array}$ & $\begin{array}{c}\text { pH } \\
\text { (stan- } \\
\text { dard } \\
\text { units) }\end{array}$ & $\begin{array}{c}\text { Dis- } \\
\text { solved } \\
\text { oxygen } \\
\text { (mg/L) }\end{array}$ & $\begin{array}{c}\text { Nitrite, } \\
\text { dis- } \\
\text { solved } \\
\text { (mg/Las } \\
\mathrm{N})\end{array}$ & $\begin{array}{c}\text { Nitrite } \\
\text { plus } \\
\text { nitrate, } \\
\text { dissolved } \\
\text { (mg/L as } \\
\mathrm{N})\end{array}$ & $\begin{array}{l}\text { Ammo- } \\
\text { nium, } \\
\text { dis- } \\
\text { solved } \\
\text { (mg/L as } \\
\mathrm{N})\end{array}$ & $\begin{array}{l}\text { Phospho- } \\
\text { rus ortho, } \\
\text { dissolved } \\
\text { (mg/L as } \mathrm{P} \text { ) }\end{array}$ \\
\hline \multicolumn{11}{|l|}{ Nebraska } \\
\hline NE03 & $09-28$ & - & 19.0 & 956 & 7.7 & 8.7 & $<.01$ & .39 & .24 & $<.01$ \\
\hline NE04 & $09-15$ & - & 15.0 & 668 & 6.7 & 2.9 & $<.01$ & 7.8 & .04 & .15 \\
\hline NE06 & $09-16$ & - & 14.0 & 356 & 7.2 & 6.0 & $<.01$ & 2.7 & .01 & .12 \\
\hline NE07 & $09-14$ & - & 13.5 & 516 & 7.4 & .3 & $<.01$ & $<.05$ & .03 & .13 \\
\hline NE08 & $09-15$ & - & 12.5 & 1090 & 7.0 & 1.4 & .01 & .44 & .05 & .05 \\
\hline NE09 & $09-15$ & - & 15.5 & 332 & 6.9 & .2 & $<.01$ & .81 & .02 & .06 \\
\hline NE10 & $09-16$ & - & 10.5 & 766 & 7.1 & .1 & $<.01$ & .84 & .05 & .02 \\
\hline NE11 & $09-23$ & - & 13.0 & 462 & 7.2 & 3.4 & $<.01$ & 2.6 & .02 & .18 \\
\hline NE13 & $09-15$ & - & 12.0 & 1040 & 7.1 & .1 & $<.01$ & $<.05$ & .37 & .06 \\
\hline NE 14 & $09-17$ & - & 12.0 & 585 & 7.1 & .2 & .02 & .29 & .17 & .15 \\
\hline NE16 & $09-15$ & - & 11.5 & 798 & 7.1 & 3.0 & $<.01$ & 6.9 & .03 & .18 \\
\hline NE17 & 09-22 & - & 13.0 & 698 & 6.1 & 6.0 & $<.01$ & 5.6 & .03 & .10 \\
\hline NE18 & $09-21$ & - & 12.0 & 738 & 7.1 & 1.0 & $<.01$ & 2.1 & .05 & .12 \\
\hline NE19 & $09-17$ & - & 12.5 & 740 & 6.8 & 7.6 & $<.01$ & 7.9 & $<.01$ & .25 \\
\hline NE20 & $09-17$ & - & 13.0 & 491 & 6.9 & 8.9 & $<.01$ & 9.5 & $<.01$ & .19 \\
\hline NE21 & $09-14$ & - & 13.0 & 492 & 7.1 & 2.1 & $<.01$ & 1.7 & .02 & .18 \\
\hline NE22 & $09-22$ & - & 12.5 & 478 & 6.8 & 5.8 & $<.01$ & 6.5 & .02 & .39 \\
\hline NE23 & $09-14$ & - & 12.5 & 860 & 7.1 & 3.9 & $<.01$ & 2.2 & .03 & .17 \\
\hline NE25 & $09-16$ & - & 12.0 & 895 & 7.1 & .2 & $<.01$ & 2.0 & 0.02 & .31 \\
\hline NE27 & $09-16$ & - & 13.5 & 992 & 7.5 & .1 & $<.01$ & .19 & .01 & .02 \\
\hline NE28 & 09-14 & - & 11.5 & 596 & 7.0 & 5.8 & $<.01$ & 3.6 & $<.01$ & .21 \\
\hline NE29 & $09-17$ & - & 12.0 & 653 & 6.8 & 5.9 & $<.01$ & 11 & .02 & .17 \\
\hline NE30 & $09-16$ & - & 11.5 & 780 & 7.2 & 1.5 & $<.01$ & 3.6 & .02 & .08 \\
\hline NE31 & $09-15$ & - & 13.0 & 891 & 7.4 & .4 & $<.01$ & 1.4 & .06 & .03 \\
\hline NE33 & $09-23$ & - & 11.5 & 1940 & 7.1 & .9 & $<.01$ & 20 & .04 & .09 \\
\hline NE34 & $09-15$ & - & 14.5 & 784 & 7.2 & .5 & $<.01$ & .33 & .11 & .06 \\
\hline NE35 & $09-14$ & - & 14.5 & 998 & 7.1 & 1.0 & $<.01$ & 5.4 & .03 & .03 \\
\hline
\end{tabular}


Table 7. Water-quality data from field measurements and laboratory analyses for inorganic nutrients, 1993—Continued

\begin{tabular}{|c|c|c|c|c|c|c|c|c|c|c|}
\hline $\begin{array}{c}\text { Site } \\
\text { identifier } \\
\text { (figs. 1-2) }\end{array}$ & $\begin{array}{c}\text { Date } \\
\text { sampled } \\
\text { (month, } \\
\text { day) }\end{array}$ & $\begin{array}{l}\text { Water } \\
\text { level } \\
\text { (feet) }\end{array}$ & $\begin{array}{l}\text { Water } \\
\text { tem- } \\
\text { pera- } \\
\text { ture } \\
\left({ }^{\circ} \mathrm{C}\right)\end{array}$ & $\begin{array}{c}\text { Specific } \\
\text { con- } \\
\text { duct- } \\
\text { ance } \\
(\mu \mathrm{S} / \mathrm{cm})\end{array}$ & $\begin{array}{c}\mathrm{pH} \\
\text { (stan- } \\
\text { dard } \\
\text { units) }\end{array}$ & $\begin{array}{c}\text { Dis- } \\
\text { solved } \\
\text { oxygen } \\
\text { (mg/L) }\end{array}$ & $\begin{array}{c}\text { Nitrite, } \\
\text { dis- } \\
\text { solved } \\
\text { (mg/L as } \\
\mathrm{N} \text { ) }\end{array}$ & $\begin{array}{c}\text { Nitrite } \\
\text { plus } \\
\text { nitrate, } \\
\text { dissolved } \\
\text { (mg/L as } \\
\mathrm{N})\end{array}$ & $\begin{array}{l}\text { Ammo- } \\
\text { nium, } \\
\text { dis- } \\
\text { solved } \\
\text { (mg/L as } \\
\mathrm{N})\end{array}$ & $\begin{array}{l}\text { Phospho- } \\
\text { rus ortho, } \\
\text { dissolved } \\
\text { (mg/L as } \mathrm{P} \text { ) }\end{array}$ \\
\hline \multicolumn{11}{|c|}{ Nebraska (Continued) } \\
\hline NE36 & $09-21$ & - & 13.5 & 538 & 6.7 & 2.4 & $<.01$ & 7.5 & .03 & .44 \\
\hline NE38 & $09-24$ & - & 13.0 & 718 & 6.9 & 4.4 & $<.01$ & 11 & .03 & .30 \\
\hline NE40 & $09-17$ & - & 13.5 & 324 & 6.9 & 7.0 & $<.01$ & 2.6 & .02 & .19 \\
\hline NE41 & $09-16$ & - & 12.5 & 783 & 7.1 & .2 & $<.01$ & .71 & .16 & .25 \\
\hline NE43 & $09-16$ & - & 11.5 & 348 & 7.3 & .1 & .03 & .26 & .23 & .14 \\
\hline NE44 & $09-15$ & - & 12.0 & 688 & 7.0 & 6.6 & $<.01$ & 7.4 & .02 & .22 \\
\hline \multicolumn{11}{|l|}{ North Dakota } \\
\hline ND01 & $10-05$ & 8.20 & 14.0 & 630 & 7.4 & .3 & $<.01$ & $<.05$ & .05 & .02 \\
\hline \multicolumn{11}{|l|}{ South Dakota } \\
\hline SD01 & $09-30$ & 4.83 & 11.5 & 820 & 7.3 & 2.4 & $<.01$ & $<.05$ & .34 & .02 \\
\hline SD02 & $09-30$ & 0.89 & 18.5 & 746 & 7.3 & .1 & $<.01$ & .19 & .14 & .06 \\
\hline SD03 & $09-30$ & 12.57 & 11.5 & 1140 & 7.4 & .1 & $<.01$ & $<.05$ & .02 & .02 \\
\hline \multicolumn{11}{|l|}{ Wisconsin } \\
\hline WI02 & $10-21$ & - & 11.0 & 174 & 7.1 & 7.5 & $<.01$ & 7.2 & .02 & .13 \\
\hline WI05 & $10-06$ & - & 11.0 & 576 & 7.4 & 8.0 & $<.01$ & 5.1 & .03 & .01 \\
\hline
\end{tabular}


Table 8. Water-quality data from laboratory analyses of water samples for selected herbicides and herbicide metabolites, 1993 [Samples were analyzed for the compounds ametryn, prometryn, propazine, and terbutryn, but these compounds were not detected above the $0.05 \mu \mathrm{g} / \mathrm{L}$ reporting limit. $\mu \mathrm{g} / \mathrm{L}$, micrograms per liter; Alachlor-ESA, alachlor ethanesulfonic acid ; <, less than]

\begin{tabular}{|c|c|c|c|c|c|c|c|c|c|c|}
\hline $\begin{array}{c}\text { Site } \\
\text { identifier } \\
\text { (figs. 1-2) }\end{array}$ & $\begin{array}{l}\text { Ala- } \\
\text { chlor } \\
(\mu g / L)\end{array}$ & $\begin{array}{c}\text { Atrazine } \\
(\mu \mathrm{g} / \mathrm{L})\end{array}$ & $\begin{array}{l}\text { Cyan- } \\
\text { azine } \\
(\mu g / L)\end{array}$ & $\begin{array}{c}\text { Deiso- } \\
\text { propyl- } \\
\text { atrazine } \\
(\mu g / L)\end{array}$ & $\begin{array}{l}\text { De- } \\
\text { ethyl- } \\
\text { atrazine } \\
(\mu g / L)\end{array}$ & $\begin{array}{c}\text { Ala- } \\
\text { chlor- } \\
\text { ESA } \\
(\mu \mathrm{g} / \mathrm{L})\end{array}$ & $\begin{array}{l}\text { Metol- } \\
\text { achlor } \\
(\mu g / L)\end{array}$ & $\begin{array}{l}\text { Metri- } \\
\text { buzin } \\
(\mu g / L)\end{array}$ & $\begin{array}{l}\text { Pro- } \\
\text { meton } \\
(\mu g / L)\end{array}$ & $\begin{array}{c}\text { Simazine } \\
(\mu g / L)\end{array}$ \\
\hline \multicolumn{11}{|l|}{ Illinois } \\
\hline IL01 & $<0.05$ & $<0.05$ & $<0.05$ & $<0.05$ & $<0.05$ & $<0.10$ & $<0.05$ & $<0.05$ & $<0.05$ & $<0.05$ \\
\hline IL03 & $<.05$ & $<.05$ & $<.05$ & $<.05$ & $<.05$ & .94 & $<.05$ & $<.05$ & $<.05$ & $<.05$ \\
\hline IL07 & $<.05$ & .37 & $<.05$ & $<.05$ & .09 & .30 & $<.05$ & $<.05$ & $<.05$ & $<.05$ \\
\hline IL09 & $<.05$ & $<.05$ & $<.05$ & $<.05$ & $<.05$ & .97 & $<.05$ & $<.05$ & $<.05$ & $<.05$ \\
\hline IL11 & $<.05$ & $<.05$ & $<.05$ & $<.05$ & .20 & 3.0 & $<.05$ & $<.05$ & $<.05$ & $<.05$ \\
\hline IL13 & $<.05$ & $<.05$ & $<.05$ & $<.05$ & $<.05$ & .86 & $<.05$ & $<.05$ & .05 & $<.05$ \\
\hline IL14 & $<.05$ & .08 & $<.05$ & $<.05$ & .12 & .60 & $<.05$ & $<.05$ & $<.05$ & $<.05$ \\
\hline IL16 & $<.05$ & $<.05$ & $<.05$ & $<.05$ & $<.05$ & $<.10$ & $<.05$ & $<.05$ & $<.05$ & $<.05$ \\
\hline IL17 & $<.05$ & $<.05$ & $<.05$ & $<.05$ & $<.05$ & .29 & $<.05$ & $<.05$ & .09 & $<.05$ \\
\hline IL19 & $<.05$ & $<.05$ & $<.05$ & $<.05$ & $<.05$ & $<.10$ & $<.05$ & $<.05$ & $<.05$ & $<.05$ \\
\hline IL20 & $<.05$ & $<.05$ & $<.05$ & $<.05$ & $<.05$ & 1.1 & $<.05$ & $<.05$ & $<.05$ & $<.05$ \\
\hline IL21 & $<.05$ & $<.05$ & $<.05$ & $<.05$ & $<.05$ & .57 & $<.05$ & $<.05$ & $<.05$ & $<.05$ \\
\hline IL22 & $<.05$ & $<.05$ & $<.05$ & $<.05$ & $<.05$ & $<.10$ & $<.05$ & $<.05$ & $<.05$ & $<.05$ \\
\hline IL25 & $<.05$ & $<.05$ & $<.05$ & .16 & $<.05$ & $<.10$ & $<.05$ & $<.05$ & .05 & $<.05$ \\
\hline IL26 & $<.05$ & $<.05$ & $<.05$ & $<.05$ & .32 & .54 & $<.05$ & $<.05$ & $<.05$ & $<.05$ \\
\hline IL27 & $<.05$ & $<.05$ & $<.05$ & $<.05$ & $<.05$ & $<.10$ & $<.05$ & $<.05$ & $<.05$ & $<.05$ \\
\hline IL30 & $<.05$ & $<.05$ & $<.05$ & $<.05$ & $<.05$ & .87 & $<.05$ & $<.05$ & $<.05$ & $<.05$ \\
\hline IL31 & $<.05$ & .21 & $<.05$ & .11 & .12 & .56 & $<.05$ & $<.05$ & $<.05$ & $<.05$ \\
\hline IL32 & $<.05$ & $<.05$ & $<.05$ & $<.05$ & $<.05$ & 1.4 & $<.05$ & $<.05$ & $<.05$ & $<.05$ \\
\hline IL34 & $<.05$ & $<.05$ & $<.05$ & $<.05$ & $<.05$ & 1.2 & $<.05$ & $<.05$ & $<.05$ & $<.05$ \\
\hline IL35 & $<.05$ & .39 & .10 & .12 & .13 & .45 & .10 & $<.05$ & $<.05$ & .05 \\
\hline IL36 & $<.05$ & $<.05$ & $<.05$ & $<.05$ & $<.05$ & $<.10$ & $<.05$ & $<.05$ & $<.05$ & $<.05$ \\
\hline IL37 & $<.05$ & $<.05$ & $<.05$ & $<.05$ & $<.05$ & .58 & $<.05$ & $<.05$ & $<.05$ & $<.05$ \\
\hline IL39 & $<.05$ & .40 & $<.05$ & $<.05$ & $<.05$ & .71 & $<.05$ & $<.05$ & $<.05$ & $<.05$ \\
\hline IL62 & $<.05$ & $<.05$ & $<.05$ & $<.05$ & $<.05$ & $<.10$ & $<.05$ & $<.05$ & $<.05$ & $<.05$ \\
\hline IL63 & $<.05$ & $<.05$ & $<.05$ & $<.05$ & $<.05$ & $<.10$ & $<.05$ & $<.05$ & $<.05$ & $<.05$ \\
\hline IL64 & $<.05$ & $<.05$ & $<.05$ & $<.05$ & $<.05$ & $<.10$ & $<.05$ & $<.05$ & $<.05$ & $<.05$ \\
\hline
\end{tabular}


Table 8. Water-quality data from laboratory analyses of water samples for selected herbicides and herbicide metabolites, 1993-Continued

\begin{tabular}{|c|c|c|c|c|c|c|c|c|c|c|}
\hline $\begin{array}{c}\text { Site } \\
\text { identifier } \\
\text { (figs. 1-2) }\end{array}$ & $\begin{array}{l}\text { Ala- } \\
\text { chlor } \\
(\mu g / L)\end{array}$ & $\begin{array}{c}\text { Atrazine } \\
(\mu g / L)\end{array}$ & $\begin{array}{l}\text { Cyan- } \\
\text { azine } \\
(\mu g / L)\end{array}$ & $\begin{array}{c}\text { Deiso- } \\
\text { propyl- } \\
\text { atrazine } \\
(\mu g / L)\end{array}$ & $\begin{array}{c}\text { De- } \\
\text { ethyl- } \\
\text { atrazine } \\
(\mu g / L)\end{array}$ & $\begin{array}{l}\text { Ala- } \\
\text { chlor- } \\
\text { ESA } \\
(\mu g / L)\end{array}$ & $\begin{array}{l}\text { Metol- } \\
\text { achlor } \\
(\mu g / L)\end{array}$ & $\begin{array}{l}\text { Metri- } \\
\text { buzin } \\
(\mu g / L)\end{array}$ & $\begin{array}{l}\text { Pro- } \\
\text { meton } \\
(\mu g / L)\end{array}$ & $\begin{array}{c}\text { Simazine } \\
(\mu g / L)\end{array}$ \\
\hline \multicolumn{11}{|l|}{ lowa } \\
\hline IA09 & $<.05$ & $<.05$ & $<.05$ & $<.05$ & $<.05$ & $<.10$ & $<.05$ & $<.05$ & $<.05$ & $<.05$ \\
\hline IA13 & $<.05$ & $<.05$ & $<.05$ & $<.05$ & $<.05$ & .55 & $<.05$ & $<.05$ & $<.05$ & $<.05$ \\
\hline IA15 & $<.05$ & .50 & $<.05$ & .21 & .31 & .51 & .59 & $<.05$ & $<.05$ & $<.05$ \\
\hline IA16 & $<.05$ & .32 & .05 & .14 & .17 & .80 & .07 & $<.05$ & $<.05$ & $<.05$ \\
\hline IA17 & $<.05$ & $<.05$ & $<.05$ & $<.05$ & $<.05$ & $<.10$ & $<.05$ & $<.05$ & $<.05$ & $<.05$ \\
\hline IA18 & $<.05$ & $<.05$ & $<.05$ & $<.05$ & $<.05$ & $<.10$ & $<.05$ & $<.05$ & $<.05$ & $<.05$ \\
\hline IA 20 & $<.05$ & .09 & $<.05$ & .13 & .09 & .35 & $<.05$ & $<.05$ & .35 & $<.05$ \\
\hline IA 21 & $<.05$ & $<.05$ & $<.05$ & $<.05$ & $<.05$ & $<.10$ & $<.05$ & $<.05$ & $<.05$ & $<.05$ \\
\hline IA22 & $<.05$ & $<.05$ & $<.05$ & $<.05$ & $<.05$ & .17 & $<.05$ & $<.05$ & $<.05$ & $<.05$ \\
\hline IA23 & $<.05$ & .07 & $<.05$ & .13 & .06 & $<.10$ & $<.05$ & $<.05$ & $<.05$ & $<.05$ \\
\hline IA24 & $<.05$ & $<.05$ & $<.05$ & $<.05$ & $<.05$ & $<.10$ & $<.05$ & $<.05$ & $<.05$ & $<.05$ \\
\hline IA25 & $<.05$ & $<.05$ & $<.05$ & $<.05$ & $<.05$ & .63 & $<.05$ & $<.05$ & .10 & $<.05$ \\
\hline IA26 & $<.05$ & $<.05$ & $<.05$ & $<.05$ & $<.05$ & $<.10$ & $<.05$ & $<.05$ & $<.05$ & $<.05$ \\
\hline IA27 & $<.05$ & $<.05$ & $<.05$ & $<.05$ & $<.05$ & $<.10$ & $<.05$ & $<.05$ & $<.05$ & $<.05$ \\
\hline IA28 & $<.05$ & $<.05$ & $<.05$ & $<.05$ & $<.05$ & $<.10$ & $<.05$ & $<.05$ & $<.05$ & $<.05$ \\
\hline IA34 & $<.05$ & $<.05$ & $<.05$ & $<.05$ & $<.05$ & .43 & $<.05$ & $<.05$ & $<.05$ & $<.05$ \\
\hline IA35 & $<.05$ & .34 & $<.05$ & .45 & .43 & .09 & $<.05$ & $<.05$ & $<.05$ & $<.05$ \\
\hline IA36 & $<.05$ & $<.05$ & $<.05$ & $<.05$ & $<.05$ & .28 & $<.05$ & $<.05$ & $<.05$ & $<.05$ \\
\hline IA37 & $<.05$ & $<.05$ & $<.05$ & $<.05$ & $<.05$ & $<.10$ & $<.05$ & $<.05$ & $<.05$ & $<.05$ \\
\hline IA38 & $<.05$ & $<.05$ & $<.05$ & $<.05$ & $<.05$ & $<.10$ & $<.05$ & $<.05$ & $<.05$ & $<.05$ \\
\hline IA40 & $<.05$ & .32 & $<.05$ & $<.05$ & .06 & .79 & .32 & $<.05$ & $<.05$ & $<.05$ \\
\hline
\end{tabular}

Kansas

KS01

ros-Coninued

$\begin{array}{llllllllll}<.05 & <.05 & <.05 & <.05 & <.05 & <.10 & <.05 & <.05 & <.05 & <.05\end{array}$

Minnesota

$\begin{array}{lllllllllll}\text { MN01 } & <.05 & <.05 & <.05 & <.05 & <.05 & <.10 & <.05 & <.05 & <.05 & <.05 \\ \text { MN03 } & <.05 & <.05 & <.05 & <.05 & <.05 & <.10 & <.05 & <.05 & <.05 & <.05\end{array}$


Table 8. Water-quality data from laboratory analyses of water samples for selected herbicides and herbicide metabolites, 1993-Continued

\begin{tabular}{|c|c|c|c|c|c|c|c|c|c|c|}
\hline $\begin{array}{c}\text { Site } \\
\text { identifier } \\
\text { (figs. 1-2) }\end{array}$ & $\begin{array}{l}\text { Ala- } \\
\text { chlor } \\
(\mu g / L)\end{array}$ & $\begin{array}{c}\text { Atrazine } \\
(\mu g / L)\end{array}$ & $\begin{array}{l}\text { Cyan- } \\
\text { azine } \\
(\mu g / L)\end{array}$ & $\begin{array}{c}\text { Deiso- } \\
\text { propyl- } \\
\text { atrazine } \\
(\mu \mathrm{g} / \mathrm{L})\end{array}$ & $\begin{array}{l}\text { De- } \\
\text { ethyl- } \\
\text { atrazine } \\
(\mu \mathrm{g} / \mathrm{L})\end{array}$ & $\begin{array}{c}\text { Ala- } \\
\text { chlor- } \\
\text { ESA } \\
(\mu g / L)\end{array}$ & $\begin{array}{l}\text { Metol- } \\
\text { achlor } \\
(\mu g / L)\end{array}$ & $\begin{array}{l}\text { Metri- } \\
\text { buzin } \\
(\mu g / L)\end{array}$ & $\begin{array}{l}\text { Pro- } \\
\text { meton } \\
(\mu g / L)\end{array}$ & $\underset{(\mu g / L)}{\text { Simazine }}$ \\
\hline \multicolumn{11}{|c|}{ Minnesota (Continued) } \\
\hline MN04 & $<.05$ & $<.05$ & $<.05$ & $<.05$ & $<.05$ & $<.10$ & $<.05$ & $<.05$ & $<.05$ & $<.05$ \\
\hline MN05 & $<.05$ & $<.05$ & $<.05$ & $<.05$ & $<.05$ & $<.10$ & $<.05$ & $<.05$ & $<.05$ & $<.05$ \\
\hline MN09 & $<.05$ & $<.05$ & $<.05$ & $<.05$ & $<.05$ & $<.10$ & $<.05$ & $<.05$ & $<.05$ & $<.05$ \\
\hline MN10 & $<.05$ & $<.05$ & $<.05$ & .48 & $<.05$ & $<.10$ & $<.05$ & $<.05$ & $<.05$ & .07 \\
\hline MN11 & $<.05$ & $<.05$ & $<.05$ & $<.05$ & $<.05$ & $<.10$ & $<.05$ & $<.05$ & $<.05$ & $<.05$ \\
\hline MN13 & $<.05$ & $<.05$ & $<.05$ & $<.05$ & $<.05$ & .71 & $<.05$ & $<.05$ & $<.05$ & $<.05$ \\
\hline MN15 & $<.05$ & $<.05$ & $<.05$ & $<.05$ & $<.05$ & .30 & $<.05$ & $<.05$ & $<.05$ & $<.05$ \\
\hline MN16 & $<.05$ & $<.05$ & $<.05$ & $<.05$ & $<.05$ & $<.10$ & $<.05$ & $<.05$ & $<.05$ & $<.05$ \\
\hline MN18 & $<.05$ & $<.05$ & $<.05$ & $<.05$ & $<.05$ & $<.10$ & $<.05$ & $<.05$ & $<.05$ & $<.05$ \\
\hline MN26 & $<.05$ & $<.05$ & $<.05$ & $<.05$ & $<.05$ & 7.3 & $<.05$ & $<.05$ & $<.05$ & $<.05$ \\
\hline MN27 & $<.05$ & $<.05$ & $<.05$ & $<.05$ & $<.05$ & 2.2 & $<.05$ & $<.05$ & $<.05$ & $<.05$ \\
\hline MN28 & $<.05$ & $<.05$ & $<.05$ & $<.05$ & $<.05$ & .11 & $<.05$ & $<.05$ & $<.05$ & $<.05$ \\
\hline
\end{tabular}

Missouri

\begin{tabular}{|c|c|c|c|c|c|c|c|c|c|c|}
\hline MO03 & $<.05$ & $<.05$ & $<.05$ & $<.05$ & $<.05$ & $<.10$ & $<.05$ & $<.05$ & $<.05$ & $<.05$ \\
\hline MO04 & $<.05$ & $<.05$ & $<.05$ & $<.05$ & $<.05$ & $<.10$ & $<.05$ & $<.05$ & $<.05$ & $<.05$ \\
\hline MO05 & .23 & $<.05$ & $<.05$ & $<.05$ & $<.05$ & $<.10$ & $<.05$ & $<.05$ & $<.05$ & $<.05$ \\
\hline MO06 & 4.3 & $<.05$ & $<.05$ & $<.05$ & $<.05$ & $<.10$ & .51 & .22 & $<.05$ & $<.05$ \\
\hline MO07 & $<.05$ & $<.05$ & $<.05$ & $<.05$ & $<.05$ & .05 & $<.05$ & .08 & $<.05$ & $<.05$ \\
\hline MO08 & $<.05$ & $<.05$ & $<.05$ & $<.05$ & $<.05$ & $<.10$ & $<.05$ & $<.05$ & $<.05$ & $<.05$ \\
\hline MO09 & .14 & $<.05$ & $<.05$ & $<.05$ & $<.05$ & .22 & $<.05$ & $<.05$ & $<.05$ & $<.05$ \\
\hline MO15 & $<.05$ & $<.05$ & $<.05$ & $<.05$ & $<.05$ & $<.10$ & $<.05$ & $<.05$ & $<.05$ & $<.05$ \\
\hline
\end{tabular}

Nebraska

$\begin{array}{lrrrrrrrrrr}\text { NE03 } & <.05 & <.05 & <.05 & <.05 & <.05 & <.10 & <.05 & <.05 & <.05 & <.05 \\ \text { NE04 } & <.05 & .22 & <.05 & <.05 & .29 & <.10 & <.05 & <.05 & .35 & <.05 \\ \text { NE06 } & <.05 & .08 & <.05 & <.05 & <.05 & <.10 & <.05 & <.05 & <.05 & <.05 \\ \text { NE07 } & <.05 & .36 & <.05 & <.05 & .08 & .11 & <.05 & <.05 & <.05 & <.05\end{array}$


Table 8. Water-quality data from laboratory analyses of water samples for selected herbicides and herbicide metabolites, 1993-Continued

\begin{tabular}{|c|c|c|c|c|c|c|c|c|c|c|}
\hline $\begin{array}{c}\text { Site } \\
\text { identifier } \\
\text { (figs. 1-2) }\end{array}$ & $\begin{array}{l}\text { Ala- } \\
\text { chlor } \\
(\mu g / L)\end{array}$ & $\begin{array}{c}\text { Atrazine } \\
(\mu \mathrm{g} / \mathrm{L})\end{array}$ & $\begin{array}{l}\text { Cyan- } \\
\text { azine } \\
(\mu g / L)\end{array}$ & $\begin{array}{l}\text { Deiso- } \\
\text { propyl- } \\
\text { atrazine } \\
(\mu g / L)\end{array}$ & $\begin{array}{c}\text { De- } \\
\text { ethyl- } \\
\text { atrazine } \\
(\mu \mathrm{g} / \mathrm{L})\end{array}$ & $\begin{array}{c}\text { Ala- } \\
\text { chlor- } \\
\text { ESA } \\
(\mu g / L)\end{array}$ & $\begin{array}{l}\text { Metol- } \\
\text { achlor } \\
(\mu g / L)\end{array}$ & $\begin{array}{l}\text { Metri- } \\
\text { buzin } \\
(\mu g / L)\end{array}$ & $\begin{array}{l}\text { Pro- } \\
\text { meton } \\
(\mu g / L)\end{array}$ & $\underset{(\mu g / L)}{\text { Simazine }}$ \\
\hline \multicolumn{11}{|c|}{ Nebraska (Continued) } \\
\hline NE08 & $<.05$ & 1.2 & $<.05$ & .11 & .73 & 1.1 & $<.05$ & $<.05$ & $<.05$ & $<.05$ \\
\hline NE09 & $<.05$ & $<.05$ & $<.05$ & $<.05$ & $<.05$ & $<.10$ & $<.05$ & $<.05$ & $<.05$ & $<.05$ \\
\hline NE10 & $<.05$ & $<.05$ & $<.05$ & $<.05$ & $<.05$ & $<.10$ & $<.05$ & $<.05$ & $<.05$ & $<.05$ \\
\hline NE11 & $<.05$ & $<.05$ & $<.05$ & $<.05$ & $<.05$ & $<.10$ & $<.05$ & $<.05$ & $<.05$ & $<.05$ \\
\hline NE13 & $<.05$ & .59 & $<.05$ & $<.05$ & .10 & 1.6 & $<.05$ & $<.05$ & $<.05$ & $<.05$ \\
\hline NE14 & $<.05$ & .62 & .45 & .09 & .10 & .43 & .08 & $<.05$ & $<.05$ & $<.05$ \\
\hline NE16 & $<.05$ & $<.05$ & $<.05$ & $<.05$ & $<.05$ & $<.10$ & $<.05$ & $<.05$ & $<.05$ & $<.05$ \\
\hline NE17 & $<.05$ & $<.05$ & $<.05$ & $<.05$ & $<.05$ & $<.10$ & $<.05$ & $<.05$ & $<.05$ & $<.05$ \\
\hline NE18 & $<.05$ & $<.05$ & $<.05$ & $<.05$ & $<.05$ & $<.10$ & $<.05$ & $<.05$ & $<.05$ & $<.05$ \\
\hline NE19 & $<.05$ & .88 & $<.05$ & .23 & .85 & $<.10$ & $<.05$ & $<.05$ & $<.05$ & $<.05$ \\
\hline NE20 & $<.05$ & $<.05$ & $<.05$ & $<.05$ & $<.05$ & $<.10$ & $<.05$ & $<.05$ & $<.05$ & $<.05$ \\
\hline NE21 & $<.05$ & $<.05$ & $<.05$ & $<.05$ & $<.05$ & $<.10$ & $<.05$ & $<.05$ & $<.05$ & $<.05$ \\
\hline NE22 & $<.05$ & $<.05$ & $<.05$ & $<.05$ & $<.05$ & $<.10$ & $<.05$ & $<.05$ & $<.05$ & $<.05$ \\
\hline NE23 & $<.05$ & $<.05$ & $<.05$ & $<.05$ & $<.05$ & .22 & $<.05$ & $<.05$ & $<.05$ & $<.05$ \\
\hline NE25 & $<.05$ & $<.05$ & $<.05$ & $<.05$ & $<.05$ & $<.10$ & $<.05$ & $<.05$ & 1.1 & $<.05$ \\
\hline NE27 & $<.05$ & .14 & $<.05$ & $<.05$ & .06 & .08 & $<.05$ & $<.05$ & $<.05$ & $<.05$ \\
\hline NE28 & $<.05$ & $<.05$ & $<.05$ & $<.05$ & $<.05$ & $<.10$ & $<.05$ & $<.05$ & $<.05$ & $<.05$ \\
\hline NE29 & $<.05$ & .36 & $<.05$ & .09 & .29 & .50 & $<.05$ & $<.05$ & $<.05$ & $<.05$ \\
\hline NE30 & $<.05$ & $<.05$ & $<.05$ & $<.05$ & $<.05$ & $<.10$ & $<.05$ & $<.05$ & $<.05$ & $<.05$ \\
\hline NE31 & $<.05$ & .06 & $<.05$ & $<.05$ & .20 & $<.10$ & $<.05$ & $<.05$ & $<.05$ & $<.05$ \\
\hline NE33 & $<.05$ & .07 & $<.05$ & $<.05$ & .30 & .83 & $<.05$ & $<.05$ & $<.05$ & $<.05$ \\
\hline NE34 & $<.05$ & $<.05$ & $<.05$ & $<.05$ & $<.05$ & $<.10$ & $<.05$ & $<.05$ & $<.05$ & $<.05$ \\
\hline NE35 & $<.05$ & .56 & $<.05$ & .25 & 1.7 & 3.0 & $<.05$ & $<.05$ & .15 & $<.05$ \\
\hline NE36 & $<.05$ & .05 & $<.05$ & $<.05$ & .06 & $<.10$ & $<.05$ & $<.05$ & $<.05$ & $<.05$ \\
\hline NE38 & $<.05$ & $<.05$ & $<.05$ & $<.05$ & $<.05$ & $<.10$ & $<.05$ & $<.05$ & $<.05$ & $<.05$ \\
\hline NE40 & $<.05$ & $<.05$ & $<.05$ & $<.05$ & $<.05$ & $<.10$ & $<.05$ & $<.05$ & $<.05$ & $<.05$ \\
\hline NE41 & $<.05$ & $<.05$ & $<.05$ & $<.05$ & $<.05$ & .06 & $<.05$ & $<.05$ & .05 & $<.05$ \\
\hline NE43 & $<.05$ & $<.05$ & $<.05$ & $<.05$ & $<.05$ & $<.10$ & $<.05$ & $<.05$ & $<.05$ & $<.05$ \\
\hline NE44 & $<.05$ & .05 & $<.05$ & $<.05$ & $<.05$ & $<.10$ & $<.05$ & $<.05$ & $<.05$ & $<.05$ \\
\hline
\end{tabular}


Table 8. Water-quality data from laboratory analyses of water samples for selected herbicides and herbicide metabolites, 1993-Continued

\begin{tabular}{|c|c|c|c|c|c|c|c|c|c|c|}
\hline $\begin{array}{c}\text { Site } \\
\text { identifier } \\
\text { (figs. 1-2) }\end{array}$ & $\begin{array}{l}\text { Ala- } \\
\text { chlor } \\
(\mu g / L)\end{array}$ & $\begin{array}{c}\text { Atrazine } \\
(\mu g / L)\end{array}$ & $\begin{array}{l}\text { Cyan- } \\
\text { azine } \\
(\mu g / L)\end{array}$ & $\begin{array}{c}\text { Deiso- } \\
\text { propyl- } \\
\text { atrazine } \\
(\mu \mathrm{g} / \mathrm{L})\end{array}$ & $\begin{array}{c}\text { De- } \\
\text { ethyl- } \\
\text { atrazine } \\
(\mu \mathrm{g} / \mathrm{L})\end{array}$ & $\begin{array}{l}\text { Ala- } \\
\text { chlor- } \\
\text { ESA } \\
(\mu \mathrm{g} / \mathrm{L})\end{array}$ & $\begin{array}{l}\text { Metol- } \\
\text { achlor } \\
(\mu g / L)\end{array}$ & $\begin{array}{l}\text { Metri- } \\
\text { buzin } \\
(\mu g / L)\end{array}$ & $\begin{array}{l}\text { Pro- } \\
\text { meton } \\
(\mu g / L)\end{array}$ & $\begin{array}{c}\text { Simazine } \\
(\mu g / L)\end{array}$ \\
\hline \multicolumn{11}{|l|}{ North Dakota } \\
\hline ND01 & $<.05$ & $<.05$ & $<.05$ & $<.05$ & $<.05$ & $<.10$ & $<.05$ & $<.05$ & $<.05$ & $<.05$ \\
\hline \multicolumn{11}{|l|}{ South Dakota } \\
\hline SD01 & .05 & $<.05$ & $<.05$ & $<.05$ & $<.05$ & $<.10$ & $<.05$ & $<.05$ & $<.05$ & $<.05$ \\
\hline SD02 & .07 & 1.8 & .88 & .32 & .42 & 1.3 & .34 & $<.05$ & $<.05$ & $<.05$ \\
\hline SD03 & $<.05$ & $<.05$ & $<.05$ & $<.05$ & $<.05$ & 1.1 & $<.05$ & $<.05$ & $<.05$ & $<.05$ \\
\hline \multicolumn{11}{|l|}{ Wisconsin } \\
\hline WI02 & $<.05$ & $<.05$ & $<.05$ & $<.05$ & $<.05$ & 2.2 & $<.05$ & $<.05$ & $<.05$ & $<.05$ \\
\hline WI05 & $<.05$ & $<.05$ & $<.05$ & $<.05$ & .13 & .43 & $<.05$ & $<.05$ & $<.05$ & $<.05$ \\
\hline
\end{tabular}


Table 9. Water-quality data from laboratory analyses of water samples for selected volatile organic compounds for wells with reported detections, $1993^{1}$

[ $\mu \mathrm{g} / \mathrm{L}$, micrograms per liter; <, less than]

\begin{tabular}{|c|c|c|c|c|c|c|c|c|}
\hline $\begin{array}{c}\text { Site } \\
\text { identifier } \\
\text { (figs. 1-2) }\end{array}$ & $\begin{array}{c}\text { 1,1,1-Tri- } \\
\text { chloro- } \\
\text { ethane } \\
(\mu \mathrm{g} / \mathrm{L})\end{array}$ & $\begin{array}{l}\text { 1,1-Di- } \\
\text { chloro- } \\
\text { ethane } \\
(\mu \mathrm{g} / \mathrm{L})\end{array}$ & $\begin{array}{c}\text { 1,2,3-Tri- } \\
\text { chloro- } \\
\text { propane } \\
(\mu \mathrm{g} / \mathrm{L})\end{array}$ & $\begin{array}{l}1,2,-\mathrm{Di}- \\
\text { chloro- } \\
\text { ethane } \\
(\mu \mathrm{g} / \mathrm{L})\end{array}$ & $\begin{array}{c}\text { Benzene } \\
(\mu \mathrm{g} / \mathrm{L})\end{array}$ & $\begin{array}{l}\text { Bromo- } \\
\text { form } \\
(\mu \mathrm{g} / \mathrm{L})\end{array}$ & $\begin{array}{c}\text { Chloro- } \\
\text { dibromo- } \\
\text { methane } \\
(\mu \mathrm{g} / \mathrm{L})\end{array}$ & $\begin{array}{l}\text { Chloro- } \\
\text { form } \\
(\mu g / L)\end{array}$ \\
\hline \multicolumn{9}{|l|}{ Illinois } \\
\hline IL01 & $<0.2$ & $<0.2$ & $<0.2$ & $<0.2$ & 0.3 & $<0.2$ & $<0.2$ & $<0.2$ \\
\hline IL07 & 1.3 & 0.4 & $<.2$ & $<.2$ & $<.2$ & $<.2$ & $<.2$ & 0.2 \\
\hline IL13 & $<.2$ & $<.2$ & $<.2$ & $<.2$ & $<.2$ & $<.2$ & $<.2$ & 1.6 \\
\hline IL26 & 0.2 & $<.2$ & $<.2$ & $<.2$ & $<.2$ & $<.2$ & $<.2$ & $<.2$ \\
\hline IL35 & $<.2$ & $<.2$ & $<.2$ & $<.2$ & $<.2$ & $<.2$ & 5.7 & 51.0 \\
\hline IL63 & $<.2$ & $<.2$ & $<.2$ & $<.2$ & $<.2$ & $<.2$ & $<.2$ & 2.4 \\
\hline \multicolumn{9}{|l|}{ lowa } \\
\hline IA 27 & $<.2$ & $<.2$ & $<.2$ & $<.2$ & $<.2$ & 1.7 & 1.6 & $<.2$ \\
\hline IA28 & $<.2$ & $<.2$ & $<.2$ & $<.2$ & $<.2$ & $<.2$ & $<.2$ & 0.3 \\
\hline
\end{tabular}

Minnesota

$\begin{array}{lrrrrrrrr}\text { MN15 } & <.2 & <.2 & <.2 & <.2 & <.2 & <.2 & <.2 & 44.0 \\ \text { MN26 } & 0.3 & 0.2 & <.2 & 0.6 & <.2 & <.2 & <.2 & <.2 \\ \text { MN28 } & <.2 & <.2 & 2.3 & 6.9 & 78.0 & <.2 & <.2 & <.2\end{array}$

Missouri

MO04

$<.2$

$<.2$

$<.2$

$<.2$

$<.2$

0.4

5.6

Nebraska

$\begin{array}{lllllllll}\mathrm{NE} 08 & <.2 & <.2 & <.2 & 0.2 & <.2 & <.2 & <.2 & <.2 \\ \mathrm{NE} 22 & <.2 & <.2 & <.2 & <.2 & <.2 & <.2 & <.2 & <.2 \\ \mathrm{NE} 34 & <.2 & 0.2 & <.2 & <.2 & <.2 & <.2 & <.2 & <.2\end{array}$

North Dakota

ND01

$<.2 \quad<.2 \quad<.2$

$<.2$

0.3

$<.2$

$<.2$

$<.2$

${ }^{1}$ Reporting limits of compounds not detected in $(\mu \mathrm{g} / \mathrm{L}): 1,1,1,2$-Tetrachloroethane $(0.2), 1,1,2,2$-Tetrachloroethane $(0.2), 1,1,2$-Trichloroethane $(0.2)$, 1,1-Dichloroethylene (0.2), 1,1-Dichloropropane (0.2), 1,2,3-Trichlorobenzene (0.2), 1,2,4-Trichlorobenzene (0.2), 1,2,4-Trimethylbenzene (0.2), 1,2Chlorotoluene (0.2), 1,2-Dibromoethane (0.2), 1,2-Dichlorobenzene (0.2), 1,2-Dichloropropane (.2), 1,2-Transdichloroethene (0.2), 1,3,5-Trimethylbenzene (0.2), 1,3-Dichlorobenzene (0.2), 1,3-Dichloropropane (0.2), 1,4-Chlorotoluene (0.2), 1,4-Dichlorobenzene (0.2), 2,2-Dichloropropane (0.2), 2-Chloro- 
Table 9. Water-quality data from laboratory analyses of water samples for selected volatile organic compounds for wells with reported detections, 1993-Continued

\begin{tabular}{|c|c|c|c|c|c|c|c|c|}
\hline $\begin{array}{c}\text { Site } \\
\text { identifier } \\
\text { (figs. 1-2) }\end{array}$ & $\begin{array}{c}\text { Dichloro- } \\
\text { bromo- } \\
\text { methane } \\
(\mu g / L)\end{array}$ & $\begin{array}{c}\text { Ethyl- } \\
\text { benzene } \\
(\mu g / L)\end{array}$ & $\begin{array}{l}\text { Methyl- } \\
\text { chloride } \\
(\mu g / L)\end{array}$ & $\begin{array}{c}\text { Methyl- } \\
\text { ene- } \\
\text { chloride } \\
(\mu g / L)\end{array}$ & $\begin{array}{l}\text { Tetra- } \\
\text { chloro- } \\
\text { ethylene } \\
(\mu g / L)\end{array}$ & $\begin{array}{c}\text { Toluene } \\
(\mu g / L)\end{array}$ & 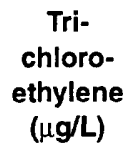 & $\begin{array}{c}\text { Xylenes } \\
(\mu g / L)\end{array}$ \\
\hline \multicolumn{9}{|l|}{ Illinois } \\
\hline IL01 & $<0.2$ & $<0.2$ & $<0.2$ & $<0.2$ & $<0.2$ & $<0.2$ & $<0.2$ & $<0.2$ \\
\hline IL07 & $<.2$ & $<.2$ & $<.2$ & $<.2$ & $<.2$ & $<.2$ & $<.2$ & .2 \\
\hline IL13 & $<.2$ & $<.2$ & $<.2$ & $<.2$ & .6 & $<.2$ & .4 & $<.2$ \\
\hline IL26 & $<.2$ & $<.2$ & $<.2$ & $<.2$ & $<.2$ & $<.2$ & $<.2$ & $<.2$ \\
\hline IL35 & 25.0 & $<.2$ & $<.2$ & $<.2$ & $<.2$ & $<.2$ & $<.2$ & $<.2$ \\
\hline IL63 & $<.2$ & $<.2$ & $<.2$ & $<.2$ & $<.2$ & $<.2$ & $<.2$ & $<.2$ \\
\hline
\end{tabular}

lowa

IA27

IA28

$.5<.2<$

$<.2<.2$

$<.2<.2 \quad<2$

$<.2<$

$<.2 \quad<.2$

$.2<.2<.2$

$<.2$

$<2$

$<.2$

$<.2$

$<.2$

Minnesota

$\begin{array}{lrrrrrrrr}\text { MN15 } & .7 & <.2 & .3 & .4 & <.2 & <.2 & <.2 & <.2 \\ \text { MN26 } & <.2 & 0.2 & <.2 & <.2 & <.2 & <.2 & <.2 & <.2 \\ \text { MN28 } & <.2 & .9 & <.2 & <.2 & <.2 & .6 & <.2 & 5.3\end{array}$

Missouri

MO04

17

$<.2 \quad<.2$

$<2$

$<.2$

$<.2$

$<.2$

Nebraska

$\begin{array}{lllllllll}\mathrm{NE} 08 & <.2 & <.2 & <.2 & <.2 & <.2 & <.2 & <.2 & <.2 \\ \mathrm{NE} 22 & <.2 & <.2 & <.2 & <.2 & <.2 & .7 & <.2 & <.2 \\ \mathrm{NE34} & <.2 & 0.2 & <.2 & <.2 & <.2 & <.2 & <.2 & <.2\end{array}$

North Dakota

ND01

$<.2$

$<.2 \quad<.2$

$<.2$

0.3

12.0

$<2$

$<.2$

ethylvinylether (1.0), Acrolein (20), Acrylonitrile (20), Bromobenzene (0.2), Bromochloromethane (0.2), Carbontetrachloride (0.2), Chlorobenzene (0.2), Chloroethane (0.2), Cis-1,2-dichloroethene (0.2), Cis-1,3-dichloropropene (0.2), Dibromochloropropane (1.0), Dibromomethane (0.2), Dichlorodifluoromethane (0.2), Trichlorotrifluoroethane (0.5), Hexachlorobutadiene (0.2), Isopropylbenzene (0.2), Methylbromide (0.2), Methyltertbutylether (1.0), NButylbenzene (0.2), N-propylbenzene (0.2), Naphthalene (0.2), P-isopropyltoluene (0.2), Sec-butylbenzene (0.2), Styrene (0.2), Tert-butylbenzene (0.2), Trans-1,3-dichloropropene (0.2), Trichlorofluoromethane (0.2), Vinyl chloride (0.2) 
Table 10. Water-quality data from field measurements and laboratory analyses for inorganic nutrients, 1994

$\left[{ }^{\circ} \mathrm{C}\right.$, degrees Celsius; $\mu \mathrm{s} / \mathrm{cm}$, microsiemens per centimeter at 25 degrees Celsius; $\mathrm{mg} / \mathrm{L}$, milligrams per liter; - , no data; $<$, less than]

\begin{tabular}{|c|c|c|c|c|c|c|c|c|c|c|}
\hline $\begin{array}{c}\text { Site } \\
\text { identifier } \\
\text { (figs. 1-2) }\end{array}$ & $\begin{array}{c}\text { Date } \\
\text { sampled } \\
\text { (month, } \\
\text { day) }\end{array}$ & $\begin{array}{l}\text { Water } \\
\text { level } \\
\text { (feet) }\end{array}$ & $\begin{array}{l}\text { Water } \\
\text { temper- } \\
\text { ature } \\
\left({ }^{\circ} \mathrm{C}\right)\end{array}$ & $\begin{array}{c}\text { Specific } \\
\text { conduc- } \\
\text { tance } \\
(\mu \mathrm{S} / \mathrm{cm})\end{array}$ & $\begin{array}{l}\mathrm{pH} \\
\text { (stan- } \\
\text { dard } \\
\text { units) }\end{array}$ & $\begin{array}{c}\text { Dis- } \\
\text { solved } \\
\text { oxygen } \\
\text { (mg/L) }\end{array}$ & $\begin{array}{l}\text { Nitrite, } \\
\text { dis- } \\
\text { solved } \\
(\mathrm{mg} / \mathrm{L} \text { as } \\
\mathrm{N})\end{array}$ & $\begin{array}{c}\text { Nitrite } \\
\text { plus } \\
\text { nitrate, } \\
\text { dissolved } \\
\text { (mg/L as } \\
\mathrm{N} \text { ) }\end{array}$ & $\begin{array}{l}\text { Ammo- } \\
\text { nium, } \\
\text { dis- } \\
\text { solved } \\
\text { (mg/L as } \\
\mathrm{N})\end{array}$ & $\begin{array}{l}\text { Phospho- } \\
\text { rus ortho, } \\
\text { dissolved } \\
\text { ( } \mathrm{mg} / \mathrm{L} \text { as } \\
\mathrm{P} \text { ) }\end{array}$ \\
\hline \multicolumn{11}{|l|}{ Illinois } \\
\hline IL09 & $7-01$ & - & 12.0 & 678 & 7.4 & 2.5 & $<0.01$ & 30 & 0.02 & $<0.01$ \\
\hline IL14 & $7-01$ & - & 11.5 & 835 & 6.8 & .0 & $<.01$ & $<.05$ & .45 & $<.01$ \\
\hline IL17 & $7-14$ & - & 12.5 & 650 & 6.9 & .1 & $<.01$ & .75 & .09 & $<.01$ \\
\hline IL26 & $7-11$ & - & 14.5 & 342 & 7.7 & 1.4 & $<.01$ & 3.1 & $<.01$ & .05 \\
\hline IL32 & $6-30$ & - & 13.0 & 482 & 7.6 & 5.1 & $<.01$ & 14 & $<.01$ & .03 \\
\hline IL34 & $6-30$ & - & 13.0 & 520 & 7.1 & .1 & $<.01$ & .45 & .14 & .02 \\
\hline IL35 & $7-12$ & - & 23.0 & 332 & 6.8 & 6.5 & $<.01$ & .94 & $<.01$ & .02 \\
\hline IL36 & $7-11$ & - & 14.5 & 800 & 6.8 & 1.0 & $<.01$ & .08 & .02 & $<.01$ \\
\hline \multicolumn{11}{|l|}{ lowa } \\
\hline IA 13 & $7-13$ & 7.00 & 11.0 & 756 & 6.9 & - & $<.01$ & $<.05$ & .14 & .01 \\
\hline IA 16 & $7-12$ & - & 13.5 & 681 & 7.1 & 1.7 & .02 & 2.2 & .04 & .11 \\
\hline IA 18 & $6-30$ & - & 11.5 & 411 & 6.2 & 9.9 & $<.01$ & $<.05$ & .45 & $<.01$ \\
\hline IA 23 & $7-01$ & - & 11.0 & 758 & 7.1 & .5 & $<.01$ & $<.05$ & .09 & $<.01$ \\
\hline IA25 & $7-19$ & - & 9.0 & 763 & 7.2 & .6 & $<.01$ & .30 & .14 & .05 \\
\hline IA 36 & $7-13$ & 17.00 & 13.0 & 641 & 7.4 & 2.8 & $<.01$ & 3.2 & .04 & .04 \\
\hline \multicolumn{11}{|l|}{ Kansas } \\
\hline KS01 & $7-26$ & - & 14.5 & 1090 & 6.7 & .3 & $<.01$ & .08 & .87 & .02 \\
\hline \multicolumn{11}{|l|}{ Minnesota } \\
\hline MN15 & $8-16$ & - & 10.0 & 1220 & 7.0 & .1 & $<.01$ & $<.05$ & .30 & .03 \\
\hline MN26 & $8-16$ & - & 14.0 & 1180 & 6.8 & .0 & $<.01$ & 17 & .10 & $<.01$ \\
\hline MN27 & $8-15$ & 9.07 & 12.5 & 1190 & 7.0 & .3 & $<.01$ & $<.05$ & .11 & $<.01$ \\
\hline MN28 & $8-16$ & - & 12.5 & 1750 & 6.9 & .0 & $<.01$ & .59 & .25 & .01 \\
\hline
\end{tabular}


Table 10. Water-quality data from laboratory analyses of water samples for selected herbicides and herbicide metabolites, 1993-Continued

\begin{tabular}{|c|c|c|c|c|c|c|c|c|c|c|}
\hline $\begin{array}{c}\text { Site } \\
\text { identifier } \\
\text { (figs. 1-2) }\end{array}$ & $\begin{array}{c}\text { Date } \\
\text { sampled } \\
\text { (month, } \\
\text { day) }\end{array}$ & $\begin{array}{l}\text { Water } \\
\text { level } \\
\text { (feet) }\end{array}$ & $\begin{array}{c}\text { Water } \\
\text { temper- } \\
\text { ature } \\
\left({ }^{\circ} \mathrm{C}\right)\end{array}$ & $\begin{array}{c}\text { Specific } \\
\text { conduc- } \\
\text { tance } \\
(\mu \mathrm{S} / \mathrm{cm})\end{array}$ & $\begin{array}{c}\text { pH } \\
\text { (stan- } \\
\text { dard } \\
\text { units) }\end{array}$ & $\begin{array}{c}\text { Dis- } \\
\text { solved } \\
\text { oxygen } \\
\text { (mg/L) }\end{array}$ & $\begin{array}{l}\text { Nitrite, } \\
\text { dis- } \\
\text { solved } \\
\text { (mg/L as } \\
\mathrm{N})\end{array}$ & $\begin{array}{l}\text { Nitrite } \\
\text { plus } \\
\text { nitrate, } \\
\text { dissolved } \\
\text { (mg/L as } \\
\mathrm{N})\end{array}$ & $\begin{array}{l}\text { Ammo- } \\
\text { nium, } \\
\text { dis- } \\
\text { solved } \\
\text { (mg/L as } \\
\mathrm{N} \text { ) }\end{array}$ & $\begin{array}{l}\text { Phospho- } \\
\text { rus ortho, } \\
\text { dissolved } \\
\text { (mg/l as } \\
\text { P) }\end{array}$ \\
\hline \multicolumn{11}{|l|}{ Missouri } \\
\hline MO04 & $6-28$ & - & 19.0 & 837 & 7.5 & .8 & .05 & .05 & $<.01$ & .01 \\
\hline MO05 & $7-22$ & 41.00 & 15.5 & 696 & 7.4 & 3.7 & $<.01$ & $<.05$ & .13 & $<.01$ \\
\hline MO06 & $7-18$ & - & 14.5 & 830 & 7.1 & .3 & $<.01$ & $<.05$ & .30 & $<.01$ \\
\hline MO07 & $7-22$ & - & 14.0 & 881 & 7.2 & 1.5 & .02 & 4.2 & .15 & .02 \\
\hline MO08 & $7-19$ & - & 7.0 & 930 & 7.2 & .2 & $<.01$ & $<.05$ & .72 & $<.01$ \\
\hline MO09 & $7-22$ & - & 15.5 & 716 & 7.2 & .2 & .02 & 3.5 & .06 & .01 \\
\hline MO15 & $6-28$ & - & 15.0 & 744 & 7.1 & .0 & $<.01$ & .05 & .20 & .02 \\
\hline \multicolumn{11}{|l|}{ Nebraska } \\
\hline NE11 & $7-29$ & - & 16.0 & 623 & 6.9 & 4.9 & $<.01$ & 6.7 & .02 & .20 \\
\hline NE14 & $7-29$ & - & 11.0 & 657 & 7.3 & .2 & .01 & .17 & .16 & .17 \\
\hline NE1 8 & $7-26$ & - & 12.0 & 714 & 7.0 & .8 & $<.01$ & 2.3 & .07 & .20 \\
\hline NE25 & $7-25$ & - & 12.0 & 937 & 7.6 & .1 & .02 & .89 & .05 & .34 \\
\hline NE28 & $7-29$ & - & 12.0 & 592 & 7.1 & 5.3 & $<.01$ & 2.9 & .03 & .22 \\
\hline NE29 & $7-28$ & - & 12.5 & 584 & 7.3 & 5.8 & $<.01$ & 10 & .03 & .17 \\
\hline NE31 & $7-25$ & - & 14.0 & 903 & 7.5 & .2 & .01 & 1.5 & .04 & .02 \\
\hline NE33 & $8-09$ & - & 14.5 & 2060 & 6.9 & .8 & $<.01$ & .13 & .02 & .05 \\
\hline NE36 & $6-28$ & - & 12.5 & 688 & 6.7 & 3.8 & $<.01$ & 15 & .04 & .42 \\
\hline \multicolumn{11}{|l|}{ South Dakota } \\
\hline SD01 & $9-02$ & 4.50 & 11.0 & 822 & 7.0 & 6.5 & $<.01$ & .29 & .09 & .02 \\
\hline SD02 & $7-12$ & 2.44 & 11.0 & 816 & 7.5 & .1 & .05 & 1.9 & .04 & .03 \\
\hline \multicolumn{11}{|l|}{ Wisconsin } \\
\hline WI02 & $6-24$ & - & 6.0 & 204 & 7.1 & 10.5 & $<.01$ & 5.5 & .02 & .13 \\
\hline
\end{tabular}


Table 11. Water-quality data from laboratory analyses of water samples for selected herbicides and herbicide metabolites, 1994

[Samples were analyzed for the compounds acetochlor, ametryn, deethylcyanazine amide, deethylcyanazine, prometryn, propazine, and terbutryn, but those compounds were not detected above the $0.05 \mu \mathrm{g} / \mathrm{L}$ reporting limit. $\mu \mathrm{g} / \mathrm{L}$, micrograms per liter; Alachlor-ESA, alachlor ethanesulfonic acid; $<$, less than]

\begin{tabular}{|c|c|c|c|c|c|c|c|c|c|c|c|}
\hline $\begin{array}{c}\text { Site } \\
\text { identifier } \\
\text { (figs. 1-2) }\end{array}$ & $\begin{array}{l}\text { Ala- } \\
\text { chlor } \\
(\mu g / L)\end{array}$ & $\begin{array}{l}\text { Atra- } \\
\text { zine } \\
(\mu g / L)\end{array}$ & $\begin{array}{l}\text { Cyan- } \\
\text { azine } \\
(\mu g / L)\end{array}$ & $\begin{array}{l}\text { Cyanzine } \\
\text { Amide } \\
(\mu g / L)\end{array}$ & $\begin{array}{l}\text { Deiso- } \\
\text { propyl- } \\
\text { atrazine } \\
(\mu \mathrm{g} / L)\end{array}$ & $\begin{array}{c}\text { De- } \\
\text { ethyl- } \\
\text { atrazine } \\
(\mu \mathrm{g} / \mathrm{L})\end{array}$ & $\begin{array}{c}\text { Ala- } \\
\text { chlor- } \\
\text { ESA } \\
(\mu \mathrm{g} / \mathrm{L})\end{array}$ & $\begin{array}{l}\text { Metol- } \\
\text { achlor } \\
(\mu \mathrm{g} / \mathrm{L})\end{array}$ & $\begin{array}{l}\text { Metri- } \\
\text { buzin } \\
(\mu g / L)\end{array}$ & $\begin{array}{l}\text { Pro- } \\
\text { meton } \\
(\mu g / L)\end{array}$ & $\begin{array}{l}\text { Sim- } \\
\text { azine } \\
(\mu g / L)\end{array}$ \\
\hline \multicolumn{12}{|l|}{ Illinois } \\
\hline IL09 & $<0.05$ & $<0.05$ & $<0.05$ & $<0.05$ & $<0.05$ & $<0.05$ & 0.8 & $<0.05$ & $<0.05$ & $<0.05$ & $<0.05$ \\
\hline IL14 & $<05$ & $<05$ & $<05$ & $<.05$ & $<.05$ & $<.05$ & .47 & $<.05$ & $<05$ & $<05$ & $<05$ \\
\hline IL17 & $<05$ & $<05$ & $<05$ & $<.05$ & $<.05$ & $<.05$ & .34 & $<.05$ & $<05$ & .06 & $<05$ \\
\hline IL26 & $<.05$ & $<05$ & $<05$ & $<.05$ & $<.05$ & .14 & .29 & $<.05$ & $<05$ & $<05$ & $<05$ \\
\hline IL32 & $<05$ & $<05$ & $<05$ & $<.05$ & $<.05$ & $<.05$ & 1.6 & $<.05$ & $<05$ & $<05$ & $<05$ \\
\hline IL34 & $<05$ & $<05$ & $<05$ & $<.05$ & $<.05$ & $<.05$ & .73 & $<.05$ & $<05$ & $<05$ & $<05$ \\
\hline IL35 & $<05$ & .65 & $<05$ & $<.05$ & $<.05$ & .05 & .90 & .17 & $<05$ & $<05$ & $<05$ \\
\hline IL36 & $<05$ & $<.05$ & $<05$ & $<.05$ & $<.05$ & $<.05$ & $<.10$ & $<.05$ & $<05$ & $<05$ & $<05$ \\
\hline
\end{tabular}

lowa

$\begin{array}{lrrrrrrrrrrr}\text { IA13 } & <05 & <.05 & <.05 & <.05 & <.05 & <.05 & .43 & <.05 & <.05 & <.05 & <.05 \\ \text { IA16 } & <05 & .78 & .06 & .47 & .14 & .14 & .33 & .19 & <.05 & <05 & <05 \\ \text { IA18 } & <05 & <.05 & <.05 & <.05 & <.05 & <.05 & <.10 & <.05 & <.05 & <.05 & <05 \\ \text { IA23 } & <.05 & <.05 & <.05 & <.05 & <.05 & <.05 & <.10 & <.05 & <.05 & <.05 & <.05 \\ \text { IA25 } & <05 & <05 & <05 & <.05 & <.05 & <.05 & 3.6 & <.05 & <.05 & .07 & <.05 \\ \text { IA36 } & <.05 & <.05 & <.05 & <.05 & <.05 & <.05 & .89 & <.05 & <.05 & <05 & <.05\end{array}$

Kansas

KS01

$<05<05<05<<.05$

$<.05<.05$

.15

$<05$

$<05<05$

Minnesota

$\begin{array}{llllllllllll}\text { MN15 } & <05 & <05 & <05 & <.05 & <.05 & <.05 & .15 & <.05 & <.05 & <05 & <05 \\ \text { MN26 } & <05 & <05 & <05 & .49 & <.05 & <.05 & 8.6 & <.05 & <05 & <05 & <05 \\ \text { MN27 } & <.05 & <05 & <05 & <.05 & <.05 & <.05 & 2.8 & <.05 & <05 & <05 & <05 \\ \text { MN28 } & <05 & <05 & <05 & <.05 & <.05 & <.05 & .29 & <.05 & <05 & <05 & <05\end{array}$


Table 11. Water-quality data from laboratory analyses of water samples for selected herbicides and herbicide metabolites, 1994-Continued

\begin{tabular}{|c|c|c|c|c|c|c|c|c|c|c|c|}
\hline $\begin{array}{c}\text { Site } \\
\text { identifier } \\
\text { (figs. 1-2) }\end{array}$ & $\begin{array}{l}\text { Ala- } \\
\text { chlor } \\
(\mu g / L)\end{array}$ & $\begin{array}{l}\text { Atra- } \\
\text { zine } \\
(\mu g / L)\end{array}$ & $\begin{array}{l}\text { Cyan- } \\
\text { azine } \\
(\mu g / L)\end{array}$ & $\begin{array}{c}\text { Cyanzine } \\
\text { Amide } \\
(\mu \mathrm{g} / \mathrm{L})\end{array}$ & $\begin{array}{c}\text { Deiso- } \\
\text { propyl- } \\
\text { atrazine } \\
(\mu \mathrm{g} / \mathrm{L})\end{array}$ & $\begin{array}{c}\text { De- } \\
\text { ethyl- } \\
\text { atrazine } \\
(\mu g / L)\end{array}$ & $\begin{array}{l}\text { Ala- } \\
\text { chlor- } \\
\text { ESA } \\
(\mu \mathrm{g} / \mathrm{L})\end{array}$ & $\begin{array}{l}\text { Metol- } \\
\text { achlor } \\
(\mu g / L)\end{array}$ & $\begin{array}{l}\text { Metri- } \\
\text { buzin } \\
(\mu g / L)\end{array}$ & $\begin{array}{l}\text { Pro- } \\
\text { meton } \\
(\mu g / L)\end{array}$ & $\begin{array}{l}\text { Sim- } \\
\text { azine } \\
(\mu g / L)\end{array}$ \\
\hline \multicolumn{12}{|l|}{ Missouri } \\
\hline MO04 & $<05$ & $<05$ & $<05$ & $<.05$ & $<.05$ & $<.05$ & $<.10$ & $<.05$ & $<05$ & $<05$ & $<05$ \\
\hline MO05 & $<05$ & $<05$ & $<05$ & $<.05$ & $<.05$ & $<.05$ & $<.10$ & $<.05$ & $<05$ & $<05$ & $<05$ \\
\hline MO06 & .40 & $<05$ & $<05$ & $<.05$ & $<.05$ & $<.05$ & .24 & $<.05$ & $<05$ & $<05$ & $<05$ \\
\hline MO07 & $<05$ & $<05$ & $<05$ & $<.05$ & $<.05$ & $<.05$ & .26 & $<.05$ & $<05$ & $<05$ & $<05$ \\
\hline MO08 & $<05$ & $<05$ & $<05$ & $<.05$ & $<.05$ & $<.05$ & $<.10$ & $<.05$ & $<05$ & $<05$ & $<05$ \\
\hline MO09 & $<05$ & $<05$ & $<05$ & $<.05$ & $<.05$ & $<.05$ & .38 & $<.05$ & $<05$ & $<05$ & $<05$ \\
\hline MO15 & $<05$ & $<05$ & $<05$ & $<.05$ & $<.05$ & $<.05$ & $<10$ & $<.05$ & $<05$ & $<05$ & $<05$ \\
\hline
\end{tabular}

Nebraska

$\begin{array}{lrrrrrrrrrrr}\mathrm{NE} 11 & <05 & .16 & <05 & <.05 & <.05 & .05 & <.01 & <.05 & <05 & <05 & <05 \\ \mathrm{NE14} & <05 & .46 & .13 & .22 & .10 & .11 & .79 & .05 & <05 & <05 & .07 \\ \mathrm{NE} 18 & <05 & <05 & <05 & <.05 & <.05 & <.05 & .11 & <.05 & <05 & <05 & <05 \\ \mathrm{NE} 25 & <05 & <05 & <05 & <.05 & <.05 & <.05 & <.10 & <.05 & <05 & .68 & <05 \\ \mathrm{NE28} & <05 & <05 & <05 & <.05 & <.05 & <.05 & <.10 & <.05 & <05 & <05 & <05 \\ \mathrm{NE29} & <05 & .12 & <05 & <.05 & <.05 & <.05 & .20 & <.05 & <05 & <05 & <05 \\ \mathrm{NE31} & <05 & <05 & <05 & <.05 & <.05 & .18 & <.10 & <.05 & <05 & <05 & <05 \\ \mathrm{NE33} & <05 & .10 & <05 & <.05 & <.05 & .28 & 1.5 & <.05 & <05 & <05 & <05 \\ \mathrm{~N} 36 & <05 & <05 & <05 & <.05 & <.05 & .05 & <.10 & <.05 & <05 & <05 & <05\end{array}$

South Dakota

$\begin{array}{lrrrrrrrrrrr}\text { SD01 } & <05 & <05 & <05 & <.05 & <.05 & <.05 & <.10 & <.05 & <05 & <05 & <05 \\ \text { SD02 } & <05 & 51 & <05 & .13 & .12 & .10 & 1.1 & <.05 & <05 & <05 & <05\end{array}$

\section{Wisconsin}

WI02

$<05 \quad<05 \quad<05 \quad<.05$

$<.05$

$<.05$

.79

$<.05$

$<05$

$<05<05$ 


\section{REFERENCES CITED}

Aga, D.S., Thurman, E.M. and Pomes, M.L. 1994, Determination of alachlor and its sulfonic acid metabolite in water by solid-phase extraction and enzyme-linked immunosorbent assay: Analytical Chemistry, v. 66, p. 1,495-1,499.

Burkart, M.R., and Kolpin, D.W., 1993, Hydrologic and land-use factors associated with herbicides and nitrate in near-surface aquifers: Journal of Environmental Quality, v. 22, p. 646-656.

Burkhardt, M.R., Cinotto, P.J., Frahm, G.W., Woodworth, M.T., and Pritt, J.W., 1995, Methods for analysis by the U.S. Geological Survey National Water Quality Laboratory-Determination of methylene blue active substances by spectrophotometry: U.S. Geological Survey Open-File Report 95-189, 16 p.

Fishman, M.J., and Friedman, L.C., eds., 1989, Methods for the determination of inorganic substances in water and fluvial sediments: U.S. Geological Survey Techniques of Water Resources Investigations, book 5, chap. A1, $545 \mathrm{p}$.

Gianessi, L.P., and Puffer, C.M., 1990, Herbicide use in the United States: Washington, D.C., Resources for the Future, Inc., Quality of the Environment Division, 127 p. [revised 1991]

Gianessi, L.P., and Puffer, C.M., 1992, Insecticide use in the United States: Washington, D.C., Resources for the Future, Inc., Quality of the Environmental Division, $209 \mathrm{p}$.

Goolsby, D.A., Scribner, E.A., Thurman, E.M., Pomes, M.L., and Meyer, M.T., 1995, Data on selected herbicides and two triazine metabolites in precipitation of the midwestern and northeastern United States, 19901991: U.S. Geological Survey Open-File Report 95$469,341 \mathrm{p}$.

Kolpin, D.W., and Burkart, M.R., 1991, Work plan for regional reconnaissance for selected herbicides and nitrate in ground water of the mid-continental United States, 1991: U.S. Geological Survey Open-File Report 91-59, $18 \mathrm{p}$.

Kolpin, D.W., Burkart, M.R., and Thurman, E.M., 1993, Hydrogeologic, water-quality, and land-use data for the reconnaissance of herbicides and nitrate in near-surface aquifers of the midcontinental United States, 1991: U.S. Geological Survey Open-File Report 93-114, $61 \mathrm{p}$.

Kolpin, D.W., Burkart, M.R., and Thurman, E.M., 1994, Herbicides and nitrate in near-surface aquifers in the Midcontinental United States, 1991: U.S. Geological Survey Water-Supply Paper 2413, 34 p.

Kolpin, D.W., Goolsby, D.A., Aga, D.S., Iverson, J.L., and Thurman, E.M., 1993, Pesticides in near-surface aquifers: Results of the midcontinental United States ground-water reconnaissance, 1991-92, in Goolsby,
D.A., and others eds., Selected papers on agricultural chemicals in water resources of the midcontinental United States, U.S. Geological Survey Open-File Report 93-418, p. 64-74.

Kolpin, D.W., and Goolsby, D.A., 1995, A regional monitoring network to investigate the occurrence and distribution of agricultural chemicals in near-surface aquifers of the midcontinental USA, in Proceedings of Conference on Groundwater Quality: Remediation and Protection, Prague, Czech Republic, May 15-18, 1995, International Association of Hydrological Sciences Publication No. 225. p. 13-20.

Kolpin, D.W., Goolsby, D.A., and Thurman, E.M., 1995, Pesticides in near-surface aquifers: An assessment using highly sensitive analytical methods and tritium: Journal of Environmental Quality, v. 24, no. 6, p. 1,1251,132 .

Kolpin, D.W., and Thurman, E.M., 1995, Postflood occurrence of selected agricultural chemicals and volatile organic compounds in near-surface unconsolidated aquifers in the Upper Mississippi River Basin, 1993: U.S. Geological Survey Circular 1120-G, 20 p.

Kolpin, D.W., Nations, B.K., Goolsby, D.A., and Thurman, E.M., 1996, Acetochlor in the hydrologic system in the Midwestern United States: Environmental Science and Technology, v. 30, n. 5, p. 1,459-1,464.

Kolpin, D.W., Thurman, E.M., and Goolsby, D.A., 1996, Occurrence of selected pesticides and their metabolites in near-surface aquifers of the midwestern United States: Environmental Science and Technology, v. 30, no. 1, p. 335-340.

Meyer, M.T., 1994, Geochemistry of cyanazine and its metabolites: Indicators of contaminant transport in surface water of the midwestern United States: University of Kansas, Lawrence, Kansas, Ph.D. thesis, 362 p.

Meyer, M.T., Mills, M.S., and Thurman, E.M., 1993, Automated solid-phase extraction of herbicides from water for gas chromatography/mass spectrometry analyses: Journal of Chromatography, v. 629, p. 55-59.

Scribner, E.A., Thurman, E.M., Goolsby, D.A., Meyer, M.T., Mills, M.S., and Pomes, M.L., 1993, Reconnaissance data for selected herbicides, two atrazine metabolites, and nitrate in surface water of the midwestern United States, 1989-90: U.S. Geological Survey Open-File Report 93-457, $77 \mathrm{p}$.

Scribner, E.A., Goolsby, D.A., Thurman, E.M., Meyer, M.T., and Pomes, M.L., 1994, Concentrations of selected herbicides, two triazine metabolites, and nutrients in storm runoff from nine stream basins in the midwestern United States, 1990-92: U.S. Geological Survey OpenFile Report 94-396, 144 p.

Thurman, E.M., Meyer, Michael, Pomes, Michael, Perry, C.A., and Schwab, A.P., 1990, Enzyme-linked immunosorbent assay compared with gas chromatography/ mass spectrometry for the determination of triazine her- 
bicides in water: Analytical Chemistry, v. 62 , no. 18 , p. 2,043-2,048.

U.S. Environmental Protection Agency, 1990, County-level fertilizer sales data: Washington, D.C., U.S. Environmental Protection Agency, Office of Policy Planning and Evaluation, September, 1990, computer disk.

1992, Drinking water regulations and health advisories: Washington, D.C., U.S. Environmental Protection Agency, Office of Water, April 1992, 13 p.

U.S. Geological Survey, 1985, National water summary - 1984 Hydrologic events, selected water-quality trends, and ground-water resources: U.S. Geological Survey Water-Supply Paper 2275, $467 \mathrm{p}$.

Wershaw, R.L., Fishman, M.J., Grahbe, R.R., and Lowe, L.E., 1987, Methods for the determination of organic substances in water and fluvial sediments: U.S. Geological Survey Techniques of Water-Resources Investigations, book 5, chap. A3.

Zaugg, S.D., Sandstrom, M.W., Smith, S.G., and Fehlberg, K.M., 1995, Methods of analysis by the U.S. Geological Survey Nation Water Quality Laboratory-Determination of pesticides in water by $\mathrm{C}-18$ solid-phase extraction and capillary-column gas chromatography/ mass spectrometry with selected-ion monitoring: U.S. Geological Survey Open-File Report 95-181, 49 p. 